\title{
Dense gas and the nature of the outflows ${ }^{\star, \star \star}$
}

\author{
I. Sepúlveda ${ }^{1}$, G. Anglada ${ }^{2}$, R. Estalella ${ }^{1}$, R. López ${ }^{1}$, J. M. Girart ${ }^{3}$, and J. Yang ${ }^{4}$ \\ 1 Departament d'Astronomia i Meteorologia, Institut de Ciències del Cosmos, Universitat de Barcelona, Martí i Franquès 1, \\ 08028 Barcelona, Catalunya, Spain \\ e-mail: inma@rocknrock.com \\ 2 Instituto de Astrofísica de Andalucía, CSIC, Camino Bajo de Huétor 50, 18008 Granada, Spain \\ 3 Institut de Ciències de l'Espai (CSIC-IEEC), Campus UAB, Facultat de Ciències, Torre C5-parell 2, 08193 Bellaterra, \\ Catalunya, Spain \\ 4 Purple Mountain Observatory, Chinese Academy of Sciences, Nanjing 210008, PR China \\ Received 17 July 2009 / Accepted 12 October 2010
}

\section{ABSTRACT}

\begin{abstract}
We present the results of the observations of the $(J, K)=(1,1)$ and the $(J, K)=(2,2)$ inversion transitions of the $\mathrm{NH}_{3} \mathrm{molecule}$ toward a large sample of 40 regions with molecular or optical outflows, using the 37 m radio telescope of the Haystack Observatory. We detected $\mathrm{NH}_{3}$ emission in 27 of the observed regions, which we mapped in 25 of them. Additionally, we searched for the $6_{16}-5_{23}$ $\mathrm{H}_{2} \mathrm{O}$ maser line toward six regions, detecting $\mathrm{H}_{2} \mathrm{O}$ maser emission in two of them, $\mathrm{HH} 265$ and AFGL 5173. We estimate the physical parameters of the regions mapped in $\mathrm{NH}_{3}$ and analyze for each particular region the distribution of high density gas and its relationship with the presence of young stellar objects. In particular, we identify the deflecting high-density clump of the HH270/110 jet. We were able to separate the $\mathrm{NH}_{3}$ emission from the L1641-S3 region into two overlapping clouds, one with signs of strong perturbation, probably associated with the driving source of the $\mathrm{CO}$ outflow, and a second, unperturbed clump, which is probably not associated with star formation. We systematically found that the position of the best candidate for the exciting source of the molecular outflow in each region is very close to an $\mathrm{NH}_{3}$ emission peak. From the global analysis of our data we find that in general the highest values of the line width are obtained for the regions with the highest values of mass and kinetic temperature. We also found a correlation between the nonthermal line width and the bolometric luminosity of the sources, and between the mass of the core and the bolometric luminosity. We confirm with a larger sample of regions the conclusion of Anglada et al. (1997) that the $\mathrm{NH}_{3}$ line emission is more intense toward molecular outflow sources than toward sources with optical outflow, suggesting a possible evolutionary scheme in which young stellar objects associated with molecular outflows progressively lose their neighboring high-density gas, weakening both the $\mathrm{NH}_{3}$ emission and the molecular outflow in the process, and making optical jets more easily detectable as the total amount of gas decreases.
\end{abstract}

Key words. ISM: jets and outflows - ISM: molecules - stars: formation

\section{Introduction}

Over the last decades, a great effort has been made to study the processes that take place in the earliest stages of stellar evolution. It is now widely accepted that low-mass stars begin their lives in the densest cores of molecular clouds and that the earliest stages of stellar evolution are associated with processes involving a strong mass loss, traced by molecular outflows, Herbig-Haro objects, and optical jets, which emanate from the deeply embedded young stellar objects. These mass-loss processes have been proposed as a way to eliminate the excess of material and of angular momentum as well as to regulate the IMF (Shu et al. 1987). The molecular outflow phase is known as one of the earliest observable phases of the stellar evolution. Several studies indicate that most, if not all, of the Class 0 and Class I sources drive molecular outflows (e.g. Davis et al. 1999) and that an important fraction of these sources are associated with Herbig-Haro objects, as well as with molecular outflows (Eiroa et al. 1994; Persi et al. 1994). These results suggest that

\footnotetext{
* Appendix A is only available in electronic form at http://www . aanda.org

$\star \star$ Haystack Observatory data as FITS files are available in electronic form at the CDS via anonymous ftp to

cdsarc.u-strasbg.fr $(130.79 .128 .5)$ or via

http://cdsarc.u-strasbg.fr/viz-bin/qcat?J/A+A/527/A41
}

both phenomena start and coexist in the early stages of the starformation process.

These outflows emanating from protostars collide with the remaining molecular cloud and disperse the surrounding material, and determine the evolution of the dense core where the star is born (Arce \& Sargent 2006). In this sense, the study of these mass-loss processes and the molecular environment of the embedded objects from which they emanate has became an important tool in order to better understand the earliest stages of stellar evolution.

Ammonia observations have proved to be a powerful tool for studying the dense cores where the stars are born. Since the first surveys of dense cores (Torrelles et al. 1983; Benson \& Myers 1989; Anglada et al. 1989), a clear link was established between dense cores, star formation and outflows (see e.g., the review of André et al. 2000). From these surveys, it was clearly established that the driving sources of outflows are usually embedded in the high-density gas, which is traced by the $\mathrm{NH}_{3}$ emission, and are located very close to the emission peak (Torrelles et al. 1983; Anglada et al. 1989). Following these results, Anglada et al. (1997, hereafter Paper I) undertook a survey of dense cores to investigate the relationship between the type of outflow and the dense gas associated with their exciting sources. A statistical study of the sources observed in that survey reveals that the ammonia emission is more intense toward 
molecular outflow sources than toward sources with only optical outflows, indicating that molecular outflows are associated with a larger amount of high-density gas. From this result, a possible evolutionary scheme was suggested in which young objects associated with molecular outflows progressively lose their neighboring high-density gas, while both the $\mathrm{NH}_{3}$ emission and the molecular outflow become weaker in the process, and the optical jets become more easily detectable as the total amount of gas and extinction decreases. In this sense, the observations of highdensity tracers, such as the $\mathrm{NH}_{3}$ molecule, confirm the decrease of high-density gas around the stars.

We present here new ammonia observations. Additional regions allow us to obtain a sample of outflow regions observed in ammonia that doubles the number used in Paper I. We selected a sample of 40 star-forming regions, taking into account the presence of molecular outflows, optical outflows, or both, and mapped with the Haystack $37 \mathrm{~m}$ telescope the $\mathrm{NH}_{3}$ emission around the suspected outflow exciting sources. In Sect. 2 we describe the observational procedure, in Sect. 3 we present the observational results (the discussion of individual sources is presented in Appendix A), in Sect. 4 we discuss the global results, in Sect. 5 we describe the relationship between the high-density gas and the nature of the outflow based on the sample, and in Sect. 6 we give our conclusions.

\section{Observations}

We observed the $(J, K)=(1,1)$ and the $(J, K)=(2,2)$ inversion transitions of the ammonia molecule with the $37 \mathrm{~m}$ radio telescope at Haystack Observatory ${ }^{1}$ in 1993 January, 1996 May and 1997 December. At the frequencies of these transitions (23.6944960 GHz and $23.7226320 \mathrm{GHz}$, respectively), the half power beam width of the telescope was 1.4 and the beam efficiency at an elevation of $40^{\circ}$ was $\sim 0.41$ for the observations made in 1993 and $\sim 0.33$ for the observations made in 1996 and 1997. In all the observing sessions, we used a cooled $K$-band maser receiver and a 5000-channel autocorrelation spectrometer with a full bandwidth of $17.8 \mathrm{MHz}$. The calibration was made with the standard noise-tube method. All spectra were corrected for elevation-dependent gain variations and for atmospheric attenuation. The rms pointing error of the telescope was $\sim 10^{\prime \prime}$. Typical system temperatures were $\sim 100 \mathrm{~K}, \sim 140 \mathrm{~K}$ and $\sim 90 \mathrm{~K}$ for the observations made in 1993, 1996, and 1997, respectively. The observations were made in the position switching mode in 1993 and 1997 and in frequency switching mode in 1996. During the data reduction the observed spectra were smoothed to a velocity resolution of $\sim 0.11 \mathrm{~km} \mathrm{~s}^{-1}$, achieving a $1 \sigma$ sensitivity of $0.2 \mathrm{~K}$ per spectral channel.

We searched for ammonia emission in the 40 regions listed in Table 1. In all cases, we first made measurements on a five-point grid centered on the position given in Table 1, with a full beam separation between points. The $\mathrm{NH}_{3}(1,1)$ line was detected in 27 sources. The $\mathrm{NH}_{3}(2,2)$ line was observed in 15 sources and was detected in 10 of them. The observed spectra of the $\mathrm{NH}_{3}(1,1)$ and $\mathrm{NH}_{3}(2,2)$ lines at the position of the emission peak are shown in Figs. 1 and 2.

In Tables 2 and 3 we give the $\mathrm{NH}_{3}(1,1)$ and $\mathrm{NH}_{3}(2,2)$ line parameters obtained from a multicomponent fit to the magnetic hyperfine structure at the position of the emission peak. The intrinsic line widths obtained range from $\sim 0.3 \mathrm{~km} \mathrm{~s}^{-1}$ to

\footnotetext{
1 Radio Astronomy at Haystack Observatory of the Northeast Radio Observatory Corporation was supported by the National Science Foundation.
}

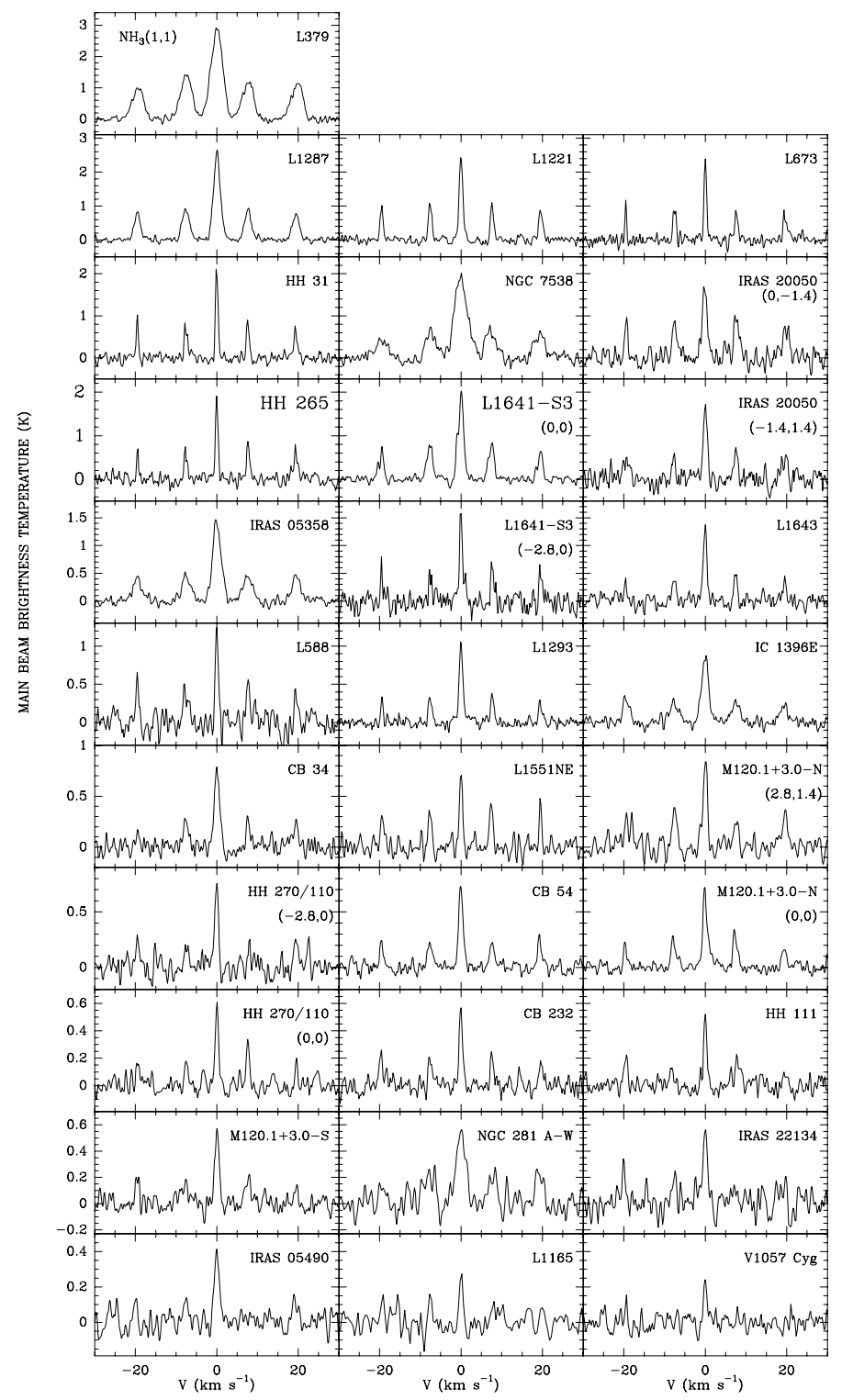

Fig. 1. Spectra of the $(J, K)=(1,1)$ inversion transition of the $\mathrm{NH}_{3}$ molecule toward the positions given in Table 2 for the detected sources. The vertical axis is the main beam brightness temperature and the horizontal axis is the velocity relative to the center of the main line (as given in Table 2). For M120.1+3.0-N, L1641-S3, HH270/110 and IRAS 20050, spectra at two different positions are shown. The spectrum of V1057 Cyg corresponds to the average of positions with detected emission in a five-point grid.

$\sim 3.6 \mathrm{~km} \mathrm{~s}^{-1}$ for the $\mathrm{NH}_{3}(1,1)$ line and from $\sim 0.5 \mathrm{~km} \mathrm{~s}^{-1}$ to $\sim 3.8$ for the $\mathrm{NH}_{3}(2,2)$ line. The values of optical depth obtained are in the range $0.1-5$ for the $\mathrm{NH}_{3}(1,1)$ and $0.1-0.5$ for the $\mathrm{NH}_{3}(2,2)$ line. We also used a single Gaussian fit to the main line to obtain the main beam brightness temperature at the position of the emission peak. The values obtained for the main beam brightness temperature for the detected sources range from $\sim 0.3 \mathrm{~K}$ to $\sim 3 \mathrm{~K}$ for the $\mathrm{NH}_{3}(1,1)$ line and from $\sim 0.2 \mathrm{~K}$ to $\sim 1 \mathrm{~K}$ for the $\mathrm{NH}_{3}(2,2)$ line.

Additionally, we searched for the $6_{16}-5_{23} \mathrm{H}_{2} \mathrm{O}$ maser line (at the frequency of $22.235080 \mathrm{GHz}$ ) toward the reference position of the six sources listed in Table 4. The $\mathrm{H}_{2} \mathrm{O}$ observations were carried out in 1996 May 16 and 17 with the same spectrometer and bandwidth used for the $\mathrm{NH}_{3}$ observations. We reached 
Table 1. Regions observed in $\mathrm{NH}_{3}$.

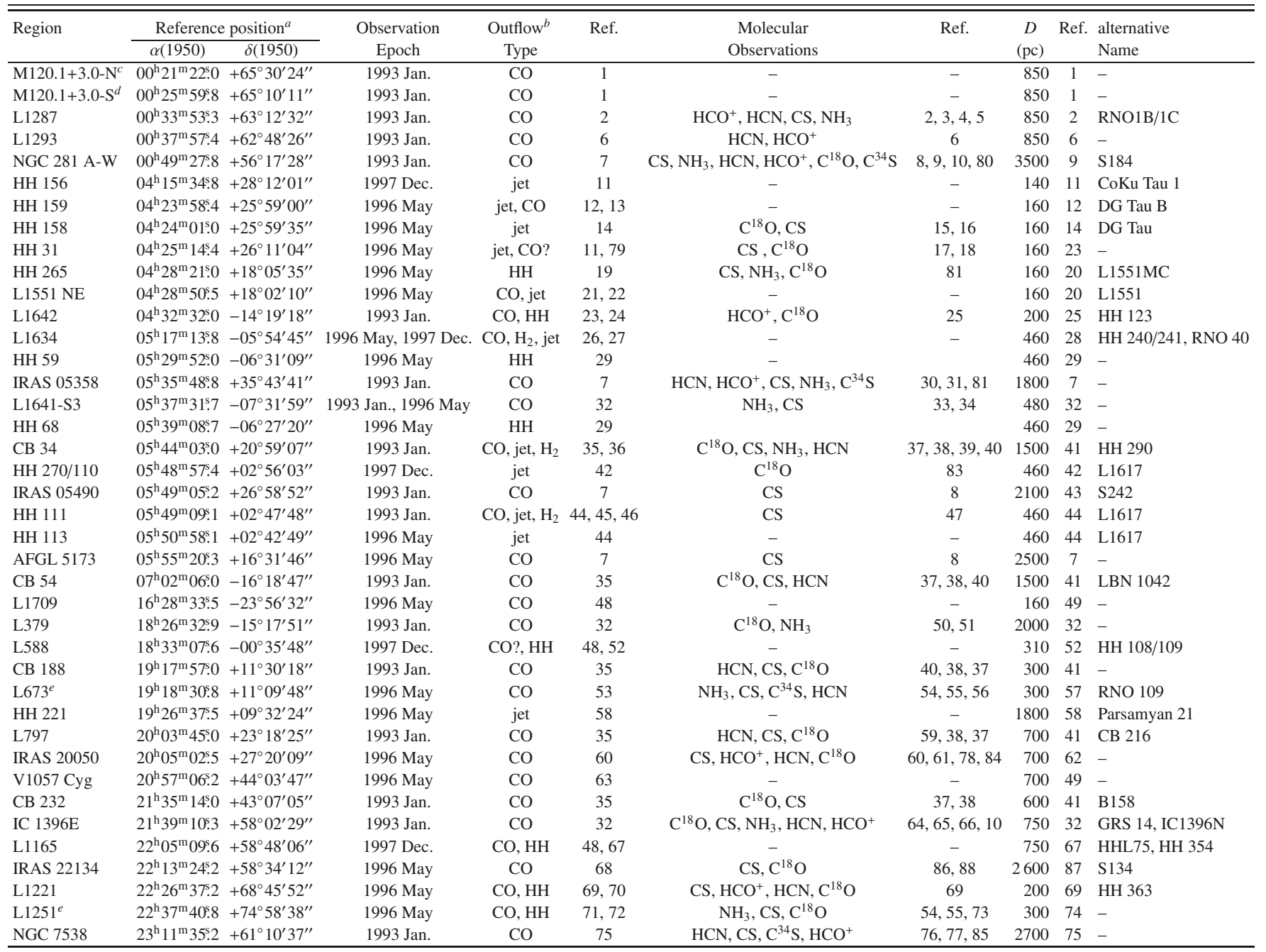

Notes. ${ }^{(a)}$ Position where the observations were centered $.{ }^{(b)} \mathrm{CO}=$ Molecular outflow; HH = Isolated Herbig-Haro object; jet = Optical outflow; $\mathrm{H}_{2}=$ Molecular hydrogen outflow. ${ }^{(c)}$ See Paper I for $\mathrm{H}_{2} \mathrm{O}$ results on IRAS $00213+6530$ in this region. ${ }^{(d)}$ See Paper $\mathrm{I}$ for $\mathrm{H}_{2} \mathrm{O}$ results on IRAS $00259+6510$ in this region. ${ }^{(e)}$ Additional $\mathrm{NH}_{3}$ data were obtained in 1990 February (see Paper I).

References. (1) Yang et al. (1990); (2) Yang et al. (1991); (3) Yang et al. (1995); (4) Estalella et al. (1993); (5) Carpenter et al. (1990); (6) Yang (1990); (7) Snell et al. (1990); (8) Carpenter et al. (1993); (9) Henning et al. (1994); (10) Cesaroni et al. (1991); (11) Strom et al. (1986); (12) Mundt et al. (1991); (13) Mitchell et al. (1994); (14) Mundt et al. (1987); (15) Hayashi et al. (1994); (16) Ohashi et al. (1991); (17) Ohashi et al. (1996); (18) Onishi et al. (1998); (19) Garnavich et al. (1992); (20) Snell (1981); (21) Moriarty-Schieven et al. (1995); (22) Devine et al. (1999); (23) Liljeström et al. (1989); (24) Reipurth \& Heathcote (1990); (25) Liljeström (1991); (26) Davis et al. (1997); (27) Hoddap \& Ladd (1995); (28) Reipurth et al. (1993); (29) Reipurth \& Graham (1988); (30) Cesaroni et al. (1999); (31) Zinchenko et al. (1997); (32) Wilking et al. (1990); (33) Harju et al. (1993); (34) Tatematsu et al. (1993); (35) Yun \& Clemens (1994)a; (36) Moreira \& Yun (1995); (37) Wang et al. (1995); (38) Launhardt et al. 1998; (39) Codella \& Scappini (1998); (40) Afonso et al. (1998); (41) Launhardt \& Henning (1997); (42) Reipurth et al. (1996); (43) Blitz et al. (1982); (44) Reipurth \& Oldberg (1991); (45) Reipurth (1989); (46) Gredel \& Reipurth (1993); (47) Yang et al. (1997); (48) Parker et al. (1991); (49) Fukui (1989); (50) Kelly \& Macdonald (1996); (51) Kelly \& Macdonald (1995); (52) Reipurth \& Eiroa (1992); (53) Armstrong \& Winnewisser (1989); (54) see Paper 1; (55) Morata et al. (1997); (56) Sandell et al. (1983); (57) Herbig \& Jones (1983); (58) Staude \& Neckel (1992); (59) Scappini et al. (1998); (60) Bachiller et al. (1995); (61) Gregersen et al. (1997); (62) Wilking et al. (1989); (63) Levreault (1988); (64) Wilking et al. (1993); (65) Serabyn et al. (1993); (66) Weikard et al. (1996); (67) Reipurth et al. (1997a); (68) Dobashi et al. (1994); (69) Umemoto et al. (1991); (70) Alten et al. (1997); (71) Sato \& Fukui (1989); (72) Eiroa et al. (1994b); (73) Sato et al. (1994); (74) Kun \& Prusti (1993); (75) Kameya et al. (1989); (76) Cao et al. (1993); (77) Kameya et al. (1986); (78) Choi et al. (1999); (79) Moriarty-Schieven et al. (1992); (80) Megeath \& Wilson (1997); (81) Swift et al. (2005); (82) Leurini et al. (2007); (83) Choi \& Tang (2006); (84) Beltrán et al. (2008); (85) Sandell et al. (2005); (86) Beuther et al. (2002b); (87) Sridharan et al. (2002); (88) Dobashi \& Uehara (2001)

a typical $(1 \sigma)$ sensitivity of $1 \mathrm{Jy}$ per spectral channel. Of the six sources observed in $\mathrm{H}_{2} \mathrm{O}$, we only detected significant $(>3 \sigma)$ $\mathrm{H}_{2} \mathrm{O}$ emission in two of them, HH 265 and AFGL 5173. The spectra of these $\mathrm{H}_{2} \mathrm{O}$ masers are shown in Fig. 3. In Table 4 we give the maser line parameters obtained from a Gaussian fit. 
Table 2. $\mathrm{NH}_{3}(1,1)$ line parameters.

\begin{tabular}{|c|c|c|c|c|c|c|c|}
\hline Region & $\begin{array}{r}\text { Position }^{a} \\
(\operatorname{arcmin})\end{array}$ & $\begin{array}{c}V_{\mathrm{LSR}^{b}} \\
\left(\mathrm{~km} \mathrm{~s}^{-1}\right)\end{array}$ & $\begin{array}{c}T_{\mathrm{MB}}(\mathrm{m})^{c} \\
\quad(\mathrm{~K})\end{array}$ & $\begin{array}{c}\Delta V^{d} \\
\left(\mathrm{~km} \mathrm{~s}^{-1}\right)\end{array}$ & $\tau_{\mathrm{m}}^{e}$ & $\begin{array}{c}A \tau_{\mathrm{m}}^{f} \\
(\mathrm{~K})\end{array}$ & $\begin{array}{c}N(1,1)^{g} \\
\left(10^{13} \mathrm{~cm}^{-2}\right)\end{array}$ \\
\hline \multirow[t]{2}{*}{$\mathrm{M} 120.1+3.0-\mathrm{N}$} & $(2.8,1.4)$ & $-18.78 \pm 0.02$ & $0.89 \pm 0.07$ & $0.90 \pm 0.05$ & $1.7 \pm 0.3$ & $1.9 \pm 0.1$ & $4.7-16.4$ \\
\hline & $(0,0)$ & $-20.23 \pm 0.01$ & $0.65 \pm 0.04$ & $1.18 \pm 0.04$ & $1.0 \pm 0.1$ & $1.06 \pm 0.05$ & $3.5-12.2$ \\
\hline M120.1+3.0-S & $(0,0)$ & $-17.41 \pm 0.02$ & $0.57 \pm 0.05$ & $1.10 \pm 0.05$ & $0.8 \pm 0.2$ & $0.83 \pm 0.07$ & $2.5-9.0$ \\
\hline L1287 & $(0,0)$ & $-17.63 \pm 0.05$ & $2.51 \pm 0.05$ & $1.77 \pm 0.01$ & $0.90 \pm 0.03$ & $3.80 \pm 0.04$ & $18.7-30.9$ \\
\hline L1293 & $(0,0)$ & $-17.67 \pm 0.01$ & $1.06 \pm 0.05$ & $0.79 \pm 0.03$ & $0.7 \pm 0.2$ & $1.7 \pm 0.1$ & $3.6-7.7$ \\
\hline NGC $281 \mathrm{~A}-\mathrm{W}$ & $(0,0)$ & $-30.32 \pm 0.04$ & $0.54 \pm 0.07$ & $2.2 \pm 0.1$ & $1.3 \pm 0.3$ & $0.93 \pm 0.07$ & $5.6-27.0$ \\
\hline HН 156 & $(0,0)$ & - & $\leq 0.2$ & - & - & - & - \\
\hline HН 159 & $(0,0)$ & - & $\leq 0.4$ & - & - & - & - \\
\hline HН 158 & $(0,0)$ & - & $\leq 0.3$ & - & - & - & - \\
\hline HH 31 & $(-7,0)$ & $+6.86 \pm 0.01$ & $2.2 \pm 0.1$ & $0.42 \pm 0.01$ & $2.6 \pm 0.2$ & $6.7 \pm 0.3$ & $7.7-16.1$ \\
\hline HH 265 & $(-1.4,1.4)$ & $+6.68 \pm 0.01$ & $1.9 \pm 0.1$ & $0.40 \pm 0.02$ & $2.5 \pm 0.3$ & $5.7 \pm 0.4$ & $6.3-14.0$ \\
\hline L1551 NE & $(0,0)$ & $+6.64 \pm 0.02$ & $0.79 \pm 0.09$ & $0.47 \pm 0.03$ & $5.0 \pm 0.8$ & $3.5 \pm 0.4$ & $4.6-22.6$ \\
\hline L1642 & $(0,0)$ & - & $\leq 0.1$ & - & - & - & - \\
\hline L1634 & $(1.4,0)$ & $+8.00 \pm 0.01$ & $1.4 \pm 0.1$ & $0.81 \pm 0.04$ & $0.6 \pm 0.2$ & $2.0 \pm 0.2$ & $4.6-8.5$ \\
\hline HH 59 & $(0,0)$ & - & $\leq 0.3$ & - & - & - & - \\
\hline IRAS 05358 & $(0,0)$ & $-16.89 \pm 0.01$ & $1.44 \pm 0.05$ & $2.32 \pm 0.03$ & $0.67 \pm 0.07$ & $1.95 \pm 0.05$ & $12.6-24.6$ \\
\hline \multirow[t]{2}{*}{ L1641-S3 } & $(0,0)$ & $+4.96 \pm 0.01$ & $2.0 \pm 0.1$ & $0.68 \pm 0.04$ & $1.3 \pm 0.3$ & $3.9 \pm 0.3$ & $7.4-14.4$ \\
\hline & $(-2.8,0)$ & $+3.76 \pm 0.01$ & $1.8 \pm 0.2$ & $0.30 \pm 0.02$ & $3.3 \pm 0.5$ & $7.0 \pm 0.3$ & $5.8-13.2$ \\
\hline HH 68 & $(0,0)$ & - & $\leq 0.3$ & - & - & - & - \\
\hline CB 34 & $(0,0)$ & $+0.72 \pm 0.02$ & $0.77 \pm 0.06$ & $1.37 \pm 0.06$ & $0.4 \pm 0.2$ & $0.99 \pm 0.07$ & $3.8-8.3$ \\
\hline \multirow[t]{2}{*}{ HH 270/110 } & $(0,0)$ & $+8.86 \pm 0.02$ & $0.67 \pm 0.06$ & $0.65 \pm 0.04$ & $1.3 \pm 0.3$ & $1.3 \pm 0.1$ & $2.4-8.9$ \\
\hline & $(-2.8,0)$ & $+8.70 \pm 0.03$ & $0.8 \pm 0.1$ & $0.8 \pm 0.1$ & $0.4 \pm 0.5$ & $1.1 \pm 0.2$ & $2.6-4.8$ \\
\hline IRAS 05490 & $(0,0)$ & $+0.78 \pm 0.03$ & $0.41 \pm 0.05$ & $1.5 \pm 0.1$ & $0.1 \pm 0.4$ & $0.45 \pm 0.02$ & $1.9-3.0$ \\
\hline HH 111 & $(0,0)$ & $+8.72 \pm 0.02$ & $0.56 \pm 0.06$ & $0.78 \pm 0.08$ & $0.4 \pm 0.3$ & $0.7 \pm 0.1$ & $1.6-3.9$ \\
\hline HH 113 & $(0,0)$ & - & $\leq 0.4$ & - & - & - & - \\
\hline AFGL 5173 & $(0,0)$ & - & $\leq 0.2$ & - & - & - & - \\
\hline CB 54 & $(0,0)$ & $+19.55 \pm 0.01$ & $0.73 \pm 0.04$ & $1.14 \pm 0.04$ & $0.8 \pm 0.2$ & $1.11 \pm 0.06$ & $3.5-10.8$ \\
\hline L1709 & $(0,0)$ & - & $\leq 0.4$ & - & - & - & - \\
\hline L379 & $(0,0)$ & $+18.89 \pm 0.01$ & $2.87 \pm 0.05$ & $2.84 \pm 0.02$ & $1.86 \pm 0.03$ & $5.98 \pm 0.05$ & $47.2-87.9$ \\
\hline L588 & $(0,0)$ & $+10.86 \pm 0.02$ & $1.3 \pm 0.1$ & $0.58 \pm 0.04$ & $2.4 \pm 0.4$ & $3.5 \pm 0.3$ & $5.6-16.4$ \\
\hline CB 188 & $(0,0)$ & - & $\leq 0.2$ & - & - & - & - \\
\hline L673 & $(0,-1.4)$ & $+7.11 \pm 0.01$ & $2.5 \pm 0.1$ & $0.41 \pm 0.1$ & $2.5 \pm 0.3$ & $7.5 \pm 0.4$ & $8.4-16.3$ \\
\hline HH 221 & $(0,0)$ & - & $\leq 0.2$ & - & - & - & - \\
\hline L797 & $(0,0)$ & - & $\leq 0.2$ & - & - & - & - \\
\hline \multirow[t]{2}{*}{ IRAS 20050} & $(0,-1.4)$ & $+6.86 \pm 0.02$ & $1.7 \pm 0.2$ & $0.93 \pm 0.04$ & $3.4 \pm 0.3$ & $5.6 \pm 0.4$ & $14.4-38.7$ \\
\hline & $(-1.4,1.4)$ & $+5.06 \pm 0.02$ & $1.8 \pm 0.2$ & $0.96 \pm 0.04$ & $0.8 \pm 0.2$ & $2.6 \pm 0.2$ & $7.1-13.1$ \\
\hline V1057 $\mathrm{Cyg}^{h}$ & $(0,0)$ & $+4.30 \pm 0.04$ & $0.3 \pm 0.1$ & $0.58 \pm 0.09$ & $\leq 3^{i}$ & $0.3-0.7^{j}$ & $0.5-14.5$ \\
\hline CB 232 & $(0,0)$ & $+12.32 \pm 0.02$ & $0.58 \pm 0.05$ & $0.68 \pm 0.04$ & $1.8 \pm 0.3$ & $1.3 \pm 0.1$ & $2.5-11.8$ \\
\hline IC $1396 \mathrm{E}$ & $(0,0)$ & $+0.53 \pm 0.02$ & $0.86 \pm 0.05$ & $1.89 \pm 0.04$ & $0.8 \pm 0.1$ & $1.22 \pm 0.05$ & $6.1-16.6$ \\
\hline L1165 & $(0,0)$ & $-1.64 \pm 0.04$ & $0.35 \pm 0.08$ & $0.6 \pm 0.1$ & $3 \pm 1$ & $0.9 \pm 0.2$ & $1.6-13.4$ \\
\hline IRAS 22134 & $(0,0)$ & $-18.62 \pm 0.04$ & $0.55 \pm 0.09$ & $1.2 \pm 0.1$ & $0.4 \pm 0.4$ & $0.7 \pm 0.1$ & $2.3-6.0$ \\
\hline L1221 & $(0,0)$ & $-4.36 \pm 0.01$ & $2.5 \pm 0.1$ & $0.71 \pm 0.01$ & $2.1 \pm 0.1$ & $6.1 \pm 0.2$ & $12.1-23.4$ \\
\hline $\mathrm{L} 1251^{k}$ & $(0,0)$ & - & $\leq 0.2$ & - & - & - & - \\
\hline NGC 7538 & $(0,-1.4)$ & $-56.22 \pm 0.02$ & $1.9 \pm 0.1$ & $3.57 \pm 0.05$ & $0.35 \pm 0.06$ & $2.32 \pm 0.06$ & $23.1-32.8$ \\
\hline
\end{tabular}

Notes. ${ }^{(a)}$ Position of the emission peak, where line parameters were obtained (in offsets from the position given in Table 1). ${ }^{(b)}$ Velocity of the line peak with respect to the local standard of rest. ${ }^{(c)}$ Main beam brightness temperature of the main line of the transition, obtained from a single Gaussian fit. For undetected sources a $3 \sigma$ upper limit is given. ${ }^{(d)}$ Intrinsic line width, obtained taking into account optical depth and hyperfine broadening, but not the spectral resolution of the spectrometer. ${ }^{(e)}$ Optical depth of the main line derived from the relative intensities of the magnetic hyperfine components. ${ }^{(f)}$ Derived from the transfer equation, where $A=f\left[J\left(T_{\mathrm{ex}}\right)-J\left(T_{\mathrm{bg}}\right)\right]$ is the "amplitude" (Pauls et al. 1983), $f$ is the filling factor, $T_{\text {ex }}$ is the excitation temperature of the transition, $T_{\mathrm{bg}}$ is the background radiation temperature and $J(T)$ is the intensity in units of temperature. Note that $A \simeq f T_{\mathrm{ex}}$, for $T_{\mathrm{ex}} \gg T_{\mathrm{bg}}{ }^{(g)}$ Beam-averaged column density for the rotational level $(1,1)$. Upper limit is obtained from

$\left[\frac{N(1,1)}{\mathrm{cm}^{-2}}\right]=1.58210^{13} \frac{\mathrm{e}^{\left(1.14 / T_{\mathrm{ex}}\right)}+1}{\mathrm{e}^{\left(1.14 / T_{\mathrm{ex}}\right)}-1} \tau_{\mathrm{m}}\left[\frac{\Delta V}{\mathrm{~km} \mathrm{~s}^{-1}}\right]$,

where $T_{\mathrm{ex}}$ is derived from the transfer equation assuming a filling factor $f=1$. If $T_{\mathrm{ex}} \gg T_{\mathrm{bg}}$ the beam averaged column density is proportional to the "amplitude" A, and the explicit dependence on $T_{\text {ex }}$ disappears, reducing to

$\left[\frac{N(1,1)}{\mathrm{cm}^{-2}}\right]=2.782 \times 10^{13}\left[\frac{A \tau_{\mathrm{m}}}{\mathrm{K}}\right]\left[\frac{\Delta V}{\mathrm{~km} \mathrm{~s}^{-1}}\right]$,

providing the lower limit for the beam-averaged column density (e.g., Ungerechts et al. 1986) . ${ }^{(h)}$ Line parameters were obtained by averaging several positions of a five-point map. ${ }^{(i)}$ Obtained by adopting a $3 \sigma$ upper limit for the intensity of the satellite lines. ${ }^{(j)}$ The highest value is obtained from the upper limit of $\tau_{\mathrm{m}}$ and the lowest value is obtained assuming optically thin emission. ${ }^{(k)}$ This region was observed and mapped in Paper I. The undetection refers to the new observed positions. 
Table 3. $\mathrm{NH}_{3}(2,2)$ line parameters.

\begin{tabular}{lccccccc}
\hline \hline Region & $\begin{array}{c}\text {Position }^{a} \\
(\operatorname{arcmin})\end{array}$ & $\begin{array}{c}V_{\mathrm{LSR}^{b}} \\
\left(\mathrm{~km} \mathrm{~s}^{-1}\right)\end{array}$ & $\begin{array}{c}T_{\mathrm{MB}}(\mathrm{m})^{c} \\
(\mathrm{~K})\end{array}$ & $\begin{array}{c}\Delta V^{d} \\
\left(\mathrm{~km} \mathrm{~s}^{-1}\right)\end{array}$ & $\begin{array}{c}\tau_{\mathrm{m}}{ }^{e} \\
\begin{array}{c}A \tau_{\mathrm{m}}{ }^{f} \\
(\mathrm{~K})\end{array}\end{array}$ & $\begin{array}{c}N(2,2)^{g} \\
\left(10^{13} \mathrm{~cm}^{-2}\right)\end{array}$ \\
\hline M120.1+3.0-N & $(2.8,1.4)$ & $-18.4 \pm 0.1$ & $0.15 \pm 0.06$ & $0.9 \pm 0.2$ & $0.2 \pm 0.1$ & $0.17 \pm 0.03$ & $0.2-0.7$ \\
L1287 & $(0,0)$ & - & $\leq 0.2$ & - & - & - & - \\
L1293 & $(0,0)$ & $-17.57 \pm 0.02$ & $0.98 \pm 0.05$ & $1.93 \pm 0.04$ & $0.27 \pm 0.04$ & $1.13 \pm 0.02$ & $2.9-4.8$ \\
HH 31 & $(0,0)$ & - & $\leq 0.2$ & - & - & - & - \\
HH 265 & $(-7,0)$ & $6.94 \pm 0.04$ & $0.24 \pm 0.06$ & $0.51 \pm 0.08$ & 0.1 & $0.27 \pm 0.04$ & $0.2-0.4$ \\
IRAS 05358 & $(-1.4,1.4)$ & - & $\leq 0.2$ & - & - & - & - \\
L1641-S3 & $(0,0)$ & $-16.79 \pm 0.04$ & $0.7 \pm 0.1$ & $2.6 \pm 0.1$ & $0.28 \pm 0.05$ & $0.83 \pm 0.02$ & $2.8-5.5$ \\
CB 34 & $(0,0)$ & $+5.1 \pm 0.1$ & $0.3 \pm 0.1$ & $1.2 \pm 0.1$ & $0.1 \pm 0.1$ & $0.30 \pm 0.03$ & $0.5-1.0$ \\
CB 54 & $(0,0)$ & - & $\leq 0.1$ & - & - & - & - \\
L379 & $(0,0)$ & - & $\leq 0.2$ & - & - & - & - \\
IRAS 20050 & $(0,0)$ & $+18.80 \pm 0.02$ & $1.3 \pm 0.1$ & $3.45 \pm 0.05$ & $0.53 \pm 0.02$ & $1.7 \pm 0.02$ & $7.7-14.3$ \\
CB 232 & $(0,0)$ & $+6.46 \pm 0.05$ & $0.7 \pm 0.1$ & $1.9 \pm 0.1$ & $0.5 \pm 0.1$ & $0.93 \pm 0.04$ & $2.3-5.5$ \\
IC 1396E & $(0,0)$ & - & $\leq 0.1$ & - & - & - & - \\
L1221 & $(0,0)$ & $+0.42 \pm 0.06$ & $0.33 \pm 0.06$ & $2.4 \pm 0.2$ & $0.23 \pm 0.06$ & $0.37 \pm 0.02$ & $1.2-3.2$ \\
NGC 7538 & $(0,0)$ & $-4.49 \pm 0.03$ & $0.5 \pm 0.1$ & $1.0 \pm 0.1$ & $0.19 \pm 0.03$ & $0.55 \pm 0.03$ & $0.7-1.4$ \\
\hline
\end{tabular}

Notes. ${ }^{(a-d, f)}$ See footnotes of Table 2. ${ }^{(e)}$ Optical depth of the $(2,2)$ main line derived from the ratio of the $(1,1)$ to $(2,2)$ antenna temperatures and the optical depth of the $(1,1)$ line, assuming the same excitation temperature for both transitions. ${ }^{(g)}$ Beam-averaged column density for the rotational level $(2,2)$. Upper limit is derived from

$\left[\frac{N(2,2)}{\mathrm{cm}^{-2}}\right]=7.469 \times 10^{12} \frac{\mathrm{e}^{\left(1.14 / T_{\mathrm{ex}}\right)}+1}{\mathrm{e}^{\left(1.14 / T_{\mathrm{ex}}\right)}-1} \tau_{\mathrm{m}}\left[\frac{\Delta V}{\mathrm{~km} \mathrm{~s}^{-1}}\right]$,

assuming that both filling factor and excitation temperature are the same for the $(1,1)$ and $(2,2)$ transitions. If $T_{\text {ex }} \gg T_{\mathrm{bg}}$, the beam-averaged column density is proportional to the "amplitude" A, and the explicit dependence on $T_{\mathrm{ex}}$ disappears, reducing to

$\left[\frac{N(2,2)}{\mathrm{cm}^{-2}}\right]=1.312 \times 10^{13}\left[\frac{A \tau_{\mathrm{m}}}{\mathrm{K}}\right]\left[\frac{\Delta V}{\mathrm{~km} \mathrm{~s}^{-1}}\right]$,

providing the lower limit for the beam-averaged column density (e.g., Ungerechts et al. 1986).

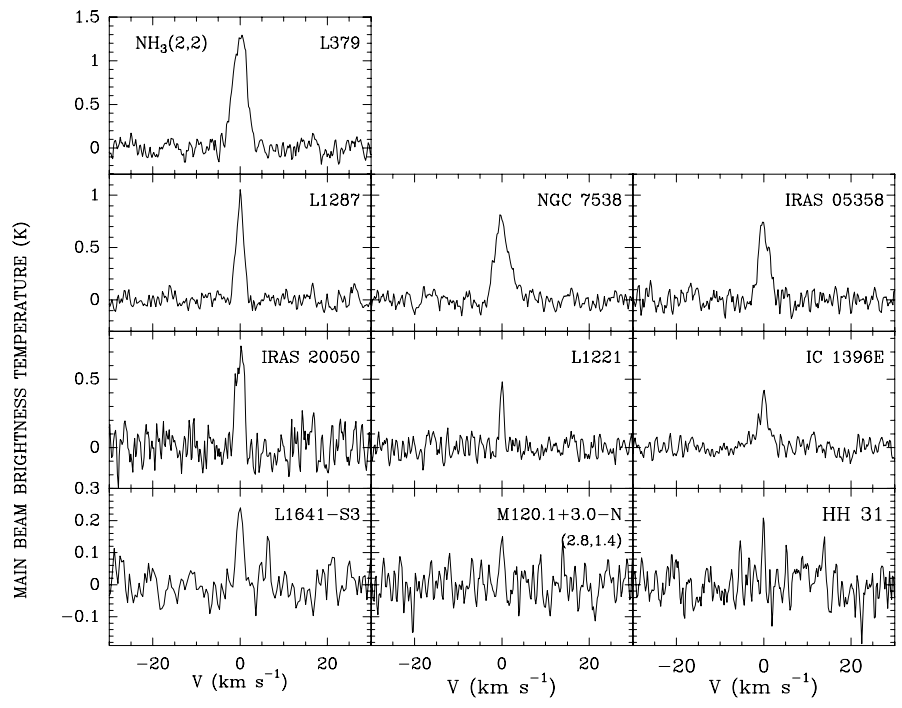

Fig. 2. Same as Fig. 1, for the $(J, K)=(2,2)$ inversion transition toward the positions given in Table 3 .

\section{Results}

In Table 5 we list the physical parameters of the molecular condensations, derived from the $\mathrm{NH}_{3}$ data given in Tables 2 and 3 , following the procedures explained in the footnotes of Table 5. We mapped the $\mathrm{NH}_{3}(1,1)$ emission in all the detected regions, except in V1057Cyg and L1551NE. Maps are shown in Figs. A.1, A.3 to A.10, A.12 to A.23 and A.25 to A.30. In
Table 4. $\mathrm{H}_{2} \mathrm{O}$ maser line parameters.

\begin{tabular}{lcccc}
\hline \hline Region & $\begin{array}{c}\text { Position }^{a} \\
(\operatorname{arcmin})\end{array}$ & $\begin{array}{c}V_{\mathrm{LSR}^{b}} \\
\left(\mathrm{~km} \mathrm{~s}^{-1}\right)\end{array}$ & $\begin{array}{c}S_{v}{ }^{c} \\
(\mathrm{Jy})\end{array}$ & $\begin{array}{c}\Delta V^{d} \\
\left(\mathrm{~km} \mathrm{~s}^{-1}\right)\end{array}$ \\
\hline M120.1+3.0-N & $(2.8,1.4)$ & - & $\leq 1$ & - \\
HH 265 & $(0,0)$ & $-9.13 \pm 0.07$ & $2.2 \pm 0.6$ & $1.1 \pm 0.2$ \\
& $(0,0)$ & $-7.14 \pm 0.07$ & $2.1 \pm 0.6$ & $0.9 \pm 0.2$ \\
L1634 & $(1.4,0)$ & - & $\leq 1.5$ & - \\
L1641-S3 & $(0,0)$ & - & $\leq 1$ & - \\
AFGL 5137 & $(0,0)$ & $+6.92 \pm 0.02$ & $11 \pm 1$ & $0.61 \pm 0.06$ \\
L1221 & $(0,0)$ & - & $\leq 1.8$ & - \\
\hline
\end{tabular}

Notes. Obtained from a Gaussian fit to the line profiles observed on May 1996.

(a) Position observed where line parameters have been obtained (in offsets from the position given in Table 1). ${ }^{(b)}$ Velocity of the line peak with respect to the local standard of rest. ${ }^{(c)}$ Flux density of the line peak. For undetected sources a $3 \sigma$ upper limit is given. ${ }^{(d)}$ Full width at half maximum.

V1057 Cyg the emission is very weak in all positions. The spectrum of this region shown in Fig. 1 and the physical parameters listed in Table 5 were obtained by averaging several points of a five-point map. In L1551NE, we detected strong $\mathrm{NH}_{3}$ emission in four positions, but we were not able to map the region (see Sect. A.8). A summary of the relevant information, taken from the literature, about the sources associated with these regions is given in Table 6.

We detected maser emission in the regions $\mathrm{HH} 265$ and AFGL 5173 (see Table 4). The position of the maser in AFGL 
A\&A 527, A41 (2011)
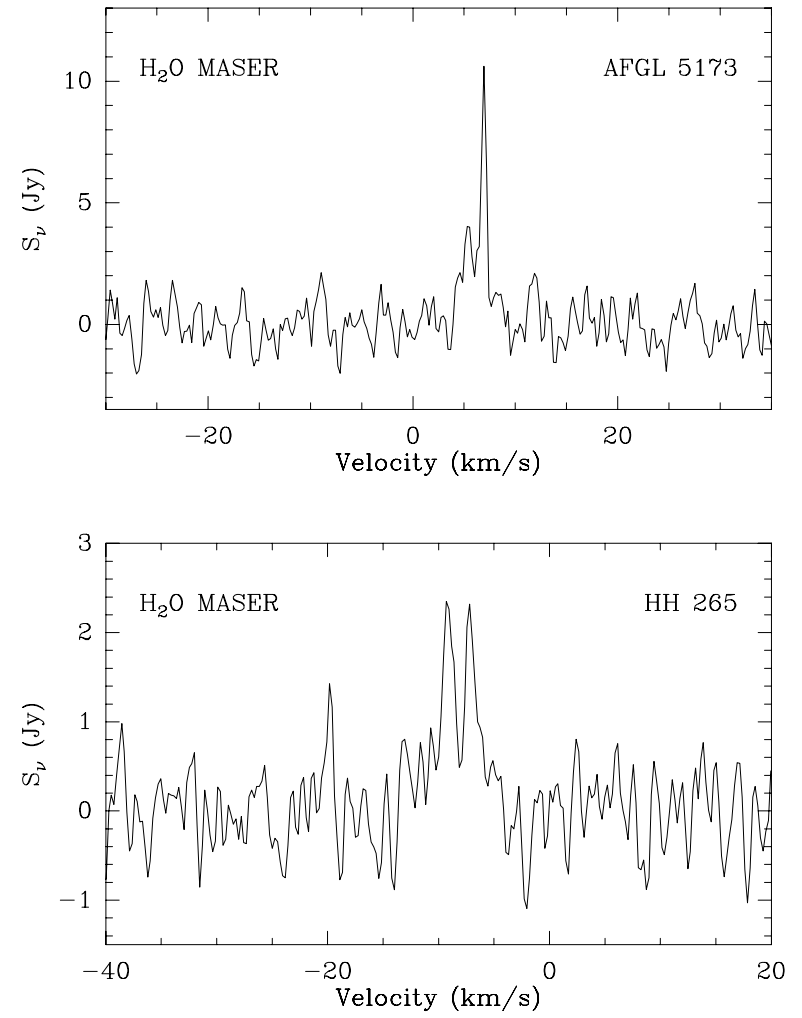

Fig. 3. Spectra of the $\mathrm{H}_{2} \mathrm{O}$ masers detected in 1996 May 16 in the regions HH 265 (bottom) and AFGL 5173 (top) toward the positions given in Table 4.

5173 coincides with that of IRAS $05553+1631$, so that the maser could be excited by the IRAS source. We detected significant maser emission in the velocity range from 6.5 to $7.2 \mathrm{~km} \mathrm{~s}^{-1}$. Brand et al. (1994) detected highly variable $\mathrm{H}_{2} \mathrm{O}$ emission toward this source between 1989 March and 1991 January in the velocity range from -7.6 to $13.1 \mathrm{~km} \mathrm{~s}^{-1}$. However no $\mathrm{NH}_{3}$ emission was detected toward the source AFGL 5173 (see Table 2). The results for individual sources are presented in Appendix A.

\section{General discussion}

\subsection{Location of the exciting sources of the outflows}

Through the $(J, K)=(1,1)$ and $(2,2)$ inversion transitions of the ammonia molecule we studied the dense gas in a sample of 40 regions with signs of star formation, as indicated by the presence of outflow activity. We detected ammonia emission in 27 regions and mapped 25 of them. This high ratio of detections $(67,5 \%)$ is a clear indication of the strong association between outflow activity and $\mathrm{NH}_{3}$ emission. This result also confirms the young nature of the powering sources of the outflows included in our sample, because they appear to be still associated with (and most of them embedded in) the dense gas from which they have been formed.

In almost all the molecular outflow regions that we mapped in $\mathrm{NH}_{3}$, the emission peaks close $(<0.1 \mathrm{pc})$ to the position of an object that was previously proposed as an outflow driving source candidate. The association with the ammonia emission peaks gives further support to the identification of these candidates as the outflow driving sources, following the criterion proposed by Anglada et al. (1989). The region IRAS 05490+2658 is the only region associated with a molecular outflow where the
$\mathrm{NH}_{3}$ emission maximum is far $(\sim 0.7 \mathrm{pc})$ from the position of the proposed exciting source; since the $\mathrm{NH}_{3}$ emission peaks close to the center of symmetry of the outflow, for this region we suggest that the exciting source could be an undetected embedded object located close to the $\mathrm{NH}_{3}$ emission maximum.

For the sources of our sample that are only associated with optical signs of outflow the $\mathrm{NH}_{3}$ emission is generally weak. Among the nine proposed exciting sources of optical outflow that we observed, only HH270 and HH290 IRS are found close to an ammonia emission peak. In all the other cases, ammonia emission is not detected or there is no known object near the ammonia maximum that could be a good candidate to drive the optical outflow.

\subsection{Physical parameters of the dense cores}

The sizes of the condensations mapped in $\mathrm{NH}_{3}$ generally range from $\sim 0.1 \mathrm{pc}$ to $\sim 1 \mathrm{pc}$. A somewhat higher value of $2 \mathrm{pc}$ is obtained for the regions NGC 281 A-W, IRAS 22134+5834 and NGC 7538, the most distant sources of our sample. We found evidence that several of the condensations mapped are elongated (as was noted by Myers et al. 1991). However, in many regions our angular resolution is not good enough to allow us to further discuss the morphology of the sources. A high angular resolution interferometric study may be relevant for the sources of our sample that appear compact in our present singledish study. Nevertheless, in several of the mapped regions (L673, M120.1+3.0-N, IRAS 20050+2720 and L1293), we can distinguish several clumps in the observed $\mathrm{NH}_{3}$ structure. In particular, in the regions M120.1+3.0-N and IRAS 20050+2720, two clumps with different velocities, but gravitationally bound, can be identified.

For the values of the kinetic temperature obtained for these regions (see Table 5), the expected thermal line widths are $\leq 0.2 \mathrm{~km} \mathrm{~s}^{-1}$. This value is significantly lower than the intrinsic line widths we have obtained, which range from 0.3 to $3.8 \mathrm{~km} \mathrm{~s}^{-1}$ (see Table 2). This result suggests that the star formation process probably introduces a significant perturbation in the molecular environment. The lower value for the intrinsic line width is found in the component at $V_{\mathrm{LSR}}=3.8 \mathrm{~km} \mathrm{~s}^{-1}$ of the L1641-S3 region, whose line widths are almost thermal. Although toward the position of the source IRAS 05375-0731 both the $3.8 \mathrm{~km} \mathrm{~s}^{-1}$ and $4.9 \mathrm{~km} \mathrm{~s}^{-1}$ ammonia components are observed, we argue in Sect. A.11 that only the broad line emission at $4.9 \mathrm{~km} \mathrm{~s}^{-1}$ is likely associated with this YSO, while no embedded sources are known to be associated with the narrow line emission at $3.8 \mathrm{~km} \mathrm{~s}^{-1}$.

The highest value of the line width is obtained for NGC 7538, which is also the region with the highest value of the mass and kinetic temperature of our sample. In general, we found that the more massive regions present higher values of the line width (see Tables 2 and 5). Also, higher values of the line width are found for the most luminous sources (see Table 6). In Fig. 4 we plot the luminosity of the sources as a function of the nonthermal line width (subtracting the thermal component using the derived rotational temperature of the region), for the sources observed in this paper and in Paper I. But because our sample of sources is limited by the sensitivity of the telescope, the most luminous sources are located mainly at larger distances than the less luminous. In order to avoid this bias of luminosity with distance, we limited our analysis to sources closer than $1 \mathrm{kpc}$. We found that the luminosity and the nonthermal line width are related by $\log \left(L_{\mathrm{bol}} / L_{\odot}\right)=(3.6 \pm 0.9) \log \left(\Delta V_{\mathrm{nth}} / \mathrm{km} \mathrm{s}^{-1}\right)+(1.8 \pm 0.2)$ with a correlation coefficient of 0.7 . A similar correlation was 
Table 5. Physical parameters of the $\mathrm{NH}_{3}$ condensations.

\begin{tabular}{|c|c|c|c|c|c|c|c|}
\hline \multirow[t]{2}{*}{ Region } & \multicolumn{2}{|c|}{ Size $^{a}$} & \multirow{2}{*}{$\begin{array}{l}T_{\text {rot }}^{b} \\
(\mathrm{~K})\end{array}$} & \multirow{2}{*}{$\begin{array}{c}N\left(\mathrm{H}_{2}\right)^{c} \\
\left(10^{22} \mathrm{~cm}^{-2}\right)\end{array}$} & \multirow{2}{*}{$\begin{array}{c}M^{d} \\
\left(M_{\odot}\right)\end{array}$} & \multirow{2}{*}{$\begin{array}{l}M_{\mathrm{vir}}^{e} \\
\left(M_{\odot}\right)\end{array}$} & \multirow{2}{*}{$\begin{array}{c}n\left(\mathrm{H}_{2}\right)^{f} \\
\left(10^{3} \mathrm{~cm}^{-3}\right)\end{array}$} \\
\hline & (arcmin) & (pc) & & & & & \\
\hline $\mathrm{M} 120.1+3.0-\mathrm{N}(2 ! 8,1 ! 4)$ & $3.9 \times 2.7$ & $0.96 \times 0.67$ & 11.2 & $1.7-6.1$ & $142-500$ & 68 & 3.4 \\
\hline $\mathrm{M} 120.1+3.0-\mathrm{N}(0,0)$ & $3.0 \times 2.0$ & $0.75 \times 0.50$ & $\leq 15$ & $\geq 0.9$ & $\geq 45$ & 89 & $\sim 2.6$ \\
\hline $\mathrm{M} 120.1+3.0-\mathrm{S}$ & $3.7 \times 2.4$ & $0.91 \times 0.59$ & $\sim 13.5$ & $0.7-2.7$ & $51-181$ & 92 & $\sim 2.8$ \\
\hline L1287 & $3.8 \times 1.9$ & $0.93 \times 0.48$ & 17.4 & $4.6-7.6$ & $259-429$ & 220 & 11.3 \\
\hline L1293 & $2.7 \times 2.0$ & $1.26 \times 0.94$ & $\leq 13$ & $\geq 1.1$ & $\geq 168$ & 71 & $\sim 7.7$ \\
\hline NGC 281 A-W & $1.9 \times 1.6$ & $1.88 \times 1.62$ & $\sim 20$ & $1 . \overline{3}-6.2$ & $505-2413$ & 871 & $\sim 1.3$ \\
\hline HH31 & $2.8 \times 2.0$ & $0.11 \times 0.08$ & 9.7 & $3.7-7.6$ & $4-9$ & 2 & 12.4 \\
\hline HH265 & $4.2 \times 2.3$ & $0.20 \times 0.11$ & $\leq 9$ & $\geq 3.3$ & $\geq 9$ & 2 & $\sim 11.3$ \\
\hline L1551 NE & $1.4 \times 1.4$ & $0.07 \times 0.07$ & $\sim 25$ & $1.0-5.1$ & $0.6-3$ & 1.6 & $\sim 1.1$ \\
\hline L1634 & $2.6 \times 2.1$ & $0.34 \times 0.28$ & $\sim 12$ & $1.6-2.9$ & $19-35$ & 21 & $\sim 13.1$ \\
\hline IRAS 05358 & $3.1 \times 1.9$ & $1.60 \times 0.98$ & 20.6 & $2.9-5.6$ & $575-1121$ & 707 & 5.9 \\
\hline $\operatorname{L} 1641-\mathrm{S} 3\left(V \sim 4.9 \mathrm{~km} \mathrm{~s}^{-1}\right)$ & $3.8 \times 2.2$ & $0.53 \times 0.31$ & 12.6 & $2.4-4.6$ & $49-96$ & 20 & 10.1 \\
\hline $\operatorname{L} 1641-\mathrm{S} 3\left(V \sim 3.8 \mathrm{~km} \mathrm{~s}^{-1}\right)$ & $5.8 \times 2.8$ & $0.81 \times 0.39$ & 13.5 & $1.7-3.9$ & $68-157$ & 5 & 6.2 \\
\hline CB 34 & $2.0 \times 1.7$ & $0.89 \times 0.76$ & $\leq 12$ & $\geq 1.3$ & $\geq 111$ & 162 & $\sim 7.8$ \\
\hline HH 270/110 ( & $1.4 \times 1.6$ & $0.19 \times 0.21$ & $\sim 13.6$ & $0 . \overline{7}-2.6$ & $3 . \overline{5}-13.3$ & 9 & $\sim 2.5$ \\
\hline $\mathrm{HH} 270 / 110(-2 ! 8,0)$ & $2.8 \times 1.7$ & $0.37 \times 0.23$ & $\sim 13.6$ & $0.8-1.4$ & $8-15$ & 22 & $\sim 10.0$ \\
\hline IRAS 05490 & $2.6 \times 1.7$ & $1.59 \times 1.05$ & $\sim 15.5$ & $0.5-0.8$ & $104-168$ & 304 & $\sim 14.3$ \\
\hline HH 111 & $1.6 \times 1.8$ & $0.22 \times 0.23$ & $\sim 13$ & $0.5-1.2$ & $3-8$ & 14 & $\sim 5.6$ \\
\hline CB 54 & $1.7 \times 1.6$ & $0.74 \times 0.68$ & $\leq 15$ & $\geq 1.0$ & $\geq 62$ & 96 & $\sim 3.3$ \\
\hline L379 & $2.0 \times 2.0$ & $1.16 \times 1.16$ & 17.8 & $11.4-21.2$ & $1948-3628$ & 982 & 7.6 \\
\hline L588 & $4.1 \times 1.9$ & $0.37 \times 0.17$ & $\sim 8$ & $4.1-11.8$ & $32-94$ & 9 & $\sim 7.5$ \\
\hline $\mathrm{L} 673(\mathrm{SE})^{g}$ & $2.2 \times 2.0$ & $0.19 \times 0.17$ & $\leq 12$ & $\geq 2.8$ & $\geq 12$ & 3 & $\sim 10.9$ \\
\hline IRAS $20050(0,-1.4)$ & $3.1 \times 2.0$ & $0.63 \times 0.41$ & 16.8 & $3.6-9.7$ & $117-315$ & 46 & 3.6 \\
\hline IRAS $20050(-1.4,1.4)$ & $3.0 \times 2.0$ & $0.61 \times 0.41$ & 16.8 & $1.8-3.3$ & $56-103$ & 49 & 8.2 \\
\hline V1057 Cyg & $1.4 \times 1.4$ & $0.29 \times 0.29$ & $\sim 10$ & $0.2-6.5$ & $2-67$ & 10 & $0.3-0.7$ \\
\hline CB 232 & $2.6 \times 2.5$ & $0.45 \times 0.43$ & $\leq 11$ & $\geq 0.9$ & $\geq 23$ & 21 & $\sim 2.2$ \\
\hline IC $1396 \mathrm{E}$ & $3.2 \times 1.9$ & $0.70 \times 0.42$ & 19.1 & $1 . \overline{4}-3.9$ & $54-145$ & 191 & 3.2 \\
\hline L1165 & $3.5 \times 2.3$ & $0.76 \times 0.49$ & $\sim 9$ & $0.9-7.3$ & $41-347$ & 23 & $\sim 1.3$ \\
\hline IRAS 22134 & $3.1 \times 2.3$ & $2.34 \times 1.74$ & $\sim 15.8$ & $0.6-1.5$ & $310-787$ & 283 & $\sim 4.3$ \\
\hline L1221 & $2.1 \times 2.0$ & $0.12 \times 0.11$ & 12.5 & $3.9-7.6$ & $7-13$ & 6 & 10.5 \\
\hline NGC 7538 & $2.5 \times 2.4$ & $1.96 \times 1.92$ & 28.3 & $5.3-7.5$ & $2514-3571$ & 2599 & 12.2 \\
\hline
\end{tabular}

Notes. ${ }^{(a)}$ Major and minor axes of the half-power contour of the $\mathrm{NH}_{3}$ emission. For sources L1551 NE and V1057 Cyg the size of the beam has been adopted. ${ }^{(b)}$ Rotational temperature, derived from the ratio of column densities in the $(1,1)$ and $(2,2)$ levels (given in Tables 1 and 2 , respectively), for the sources where the $(2,2)$ line was detected. For the sources undetected in the $(2,2)$ line, an upper limit was obtained assuming optically thin emission. For sources not observed in the $(2,2)$ line, we assumed that $T_{\mathrm{ex}}(\mathrm{CO})=T_{\mathrm{rot}}(22-11)=T_{k}$, where the CO data are from Yang et al. (1990) (M120.1+3.0-S), Henning et al. 1994 (NGC 281 A-W), Moriarty-Schieven et al. (1995) (L1551 NE), Reipurth \& Oldberg (1991) (HH 270/110 and HH 111), Snell et al. (1990) (IRAS 05490), Parker et al. (1991) (L588 and L1165), Levreault (1988) (V1057 Cyg) and Dobashi et al. (1994) (IRAS 22134). For L1634, $T_{\text {rot }}=12 \mathrm{~K}$ has been adopted. ${ }^{(c)}$ Beam-averaged $\mathrm{H}_{2}$ column density, obtained from the $\mathrm{NH}_{3}$ column density adopting an $\mathrm{NH}_{3}$ abundance of $\left[\mathrm{NH}_{3} / \mathrm{H}_{2}\right]=10^{-8}$ (see Anglada et al. 1995, for a discussion on $\mathrm{NH}_{3}$ abundances). The $\mathrm{NH}_{3}$ column density is obtained assuming that only the rotational metastable levels of the $\mathrm{NH}_{3}$ are significantly populated at their LTE ratios corresponding to $T_{k}=T_{R}(22-11) .{ }^{(d)}$ Mass of the condensation, derived from the beam-averaged $\mathrm{H}_{2}$ column density and the observed area. ${ }^{(e)}$ Virial mass obtained from $\left[M_{\mathrm{vir}} / M_{\odot}\right]=210[R / \mathrm{pc}]\left[\Delta V / \mathrm{km} \mathrm{s}^{-1}\right]^{2}$, where $R$ is the radius of the clump, taken as half the geometrical mean of the major and minor axes, and $\Delta V$ is the intrinsic line width given in Table 2. ${ }^{(f)}$ Volume density, derived from the two-level model (Ho \& Townes 1983). ${ }^{(g)}$ Parameters of the southeastern clump. Parameters of the northwestern clump are given in Paper I .

found by Jijina et al. (1999). This correlation indicates that the most luminous sources produce a large perturbation in the surrounding material.

We also note that regions associated with $\mathrm{CO}$ molecular outflow have mostly higher values of the line width than regions with optical outflow only.

Partly because of our lack of angular resolution, we are not able to measure the velocity gradient in our regions in detail. However, it is remarkable that in L1287 our results show a strong velocity gradient with sudden velocity shifts of up to $\sim 1 \mathrm{~km} \mathrm{~s}^{-1}$ between contiguous positions. A high angular resolution VLA study of this region (Sepúlveda et al., in preparation) shows that this region also exhibits a complex kinematics at small scale. Moreover, in two cases (M120.1+3.0-N and IRAS 20050+2720) the observed velocity distribution is compatible with two clumps that are gravitationally bound. The region L1641-S3 exhibits two velocity components separated by $\sim 1 \mathrm{~km} \mathrm{~s}^{-1}$ that we interpreted as two distinct clumps of emission.

The $\mathrm{H}_{2}$ column densities we obtained are generally $\sim 10^{22} \mathrm{~cm}^{-2}$ (assuming $\left[\mathrm{NH}_{3} / \mathrm{H}_{2}\right]=10^{-8}$ ), implying mean visual extinctions of $\sim 10 \mathrm{mag}$. For L379 we obtained the highest $\mathrm{H}_{2}$ column density $\left(\sim 10^{23} \mathrm{~cm}^{-2}\right.$, corresponding to a visual extinction of $\sim 100 \mathrm{mag}$ ), suggesting that this object is very deeply embedded.

The masses we obtained for the observed regions cover a wide range of values, from 1 to $3000 M_{\odot}$ (the highest value corresponds to the NGC 7538 region). Most of the sources of our sample are low-mass objects, and the values of the mass 
Table 6. Summary of properties of relevant sources in the regions detected in $\mathrm{NH}_{3}$

\begin{tabular}{|c|c|c|c|c|c|c|c|c|c|c|c|}
\hline Region & IRAS & $\begin{array}{l}L_{\text {bol }} \\
\left(L_{\odot}\right)\end{array}$ & Ref. & $\begin{array}{c}\text { Evolutionary } \\
\text { status }\end{array}$ & Ref. & $\begin{array}{c}\text { Detection at } \\
\text { other wavelengths }\end{array}$ & Ref. & $\begin{array}{c}\mathrm{H}_{2} \mathrm{O} \\
\text { maser? }\end{array}$ & Ref. & $\begin{array}{l}\text { Outflow } \\
\text { source? }\end{array}$ & Ref. \\
\hline \multirow[t]{2}{*}{$\mathrm{M} 120.1+3.0-\mathrm{N}$} & $00213+6530$ & 12.9 & 1 & - & - & $\mathrm{mm}, \mathrm{cm}$ & 109 & Yes & 2 & Yes & 1 \\
\hline & $00217+6533$ & 12.0 & 1 & - & - & - & - & - & - & $?$ & 3 \\
\hline \multirow[t]{2}{*}{$\mathrm{M} 120.1+3.0-\mathrm{S}$} & $00259+6510$ & 9.9 & 1 & - & - & - & - & No & 4 & Yes & 1 \\
\hline & $00256+6511$ & 20.3 & 1 & - & - & - & - & - & - & $?$ & 3 \\
\hline L1287 & $00338+6312$ & 1800 & 5 & Class I ${ }^{a}$ & 6 & NIR, MIR, smm, mm, cm & $7,95,8,9,10$ & Yes & 6 & $?$ & 11 \\
\hline L1293 & $00379+6248$ & $<21$ & 12 & - & - & - & - & Yes & 13 & Yes & 12 \\
\hline NGC $281 \mathrm{~A}-\mathrm{W}$ & $00494+5617$ & 8790 & 14 & $-^{a}$ & - & NIR, FIR, mm & $7,15,14$ & Yes & 16 & Yes & 11 \\
\hline HH 31 & $04248+2612$ & 0.36 & 17 & Class I & 17 & NIR, FIR, smm, mm & $17,18,19,20$ & No. & 13 & Yes & 21 \\
\hline L1551 NE & $04288-1802$ & 3.9 & 22 & Class 0 & 23 & $\mathrm{NIR}, \mathrm{smm}, \mathrm{mm}, \mathrm{cm}$ & $24,19,20,25$ & - & - & Yes & 23 \\
\hline \multirow[t]{2}{*}{ L1634 } & $05173-0555$ & 17 & 26 & Class 0 & 27 & NIR, FIR, smm, mm, cm & $28,18,29,24,27$ & No & 30 & Yes & 28 \\
\hline & $\operatorname{IRS} 7^{b}$ & $0.03^{c}$ & 27 & Class I/0 & 27 & NIR, smm & 28,27 & - & - & Yes & 28 \\
\hline IRAS 05358 & $05358+3543$ & 6300 & 31 & Herbig $\mathrm{Ae} / \mathrm{Be}^{a}{ }^{a}$ & 32 & NIR, MIR, smm, mm & $33,32,104,96,31$ & Yes & 34 & Yes & 11 \\
\hline L1641-S3 & $05375-0731$ & 100 & 35 & Class I & 36 & NIR, FIR, smm, mm, cm & $36,37,29,35,38$ & Yes & 39 & Yes & 40 \\
\hline CB 34 & $05440+2059$ & 130 & 41 & Class I & 42 & NIR, smm, mm, cm & $42,43,41,44$ & Yes & 58 & Yes & 45 \\
\hline \multirow[t]{2}{*}{ HH 270/110 } & $05487+0255$ & 7 & 46 & Class I & 46 & NIR, FIR, cm & $47,46,48$ & No & 49 & Yes & 46 \\
\hline & $05489+0256$ & 5.3 & 50 & Class I & 50 & $\mathrm{NIR}, \mathrm{mm}, \mathrm{cm}$ & $50,97,48$ & - & - & Yes & 50 \\
\hline IRAS 05490 & $05490+2658$ & 4200 & 11 & Class I $?^{a}$ & 7 & NIR, FIR, cm & 7,15 & No & 16 & Yes & 11 \\
\hline HH 111 & $05491+0247$ & 25 & 46 & Class 0 & 52 & $\mathrm{NIR}, \mathrm{smm}, \mathrm{mm}, \mathrm{cm}$ & $53,26,54,55$ & No & 30 & Yes & 46 \\
\hline CB 54 & 07020-1618 & 400 & 43 & Class I & 41 & NIR, MIR, smm, mm, cm & $56,105,42,43,57$ & Yes & 58 & Yes & 45 \\
\hline L379 & $18265-1517$ & 16000 & 59 & $-^{a}$ & - & $\mathrm{smm}, \mathrm{mm}, \mathrm{cm}$ & 59,8 & Yes & 60 & Yes & 40 \\
\hline L588 & $18331-0035$ & 3.7 & 26 & Class I & 61 & NIR, mm & 103,61 & - & - & $?$ & 61 \\
\hline \multirow[t]{2}{*}{ IRAS 20050} & $20050+2720$ & 206 & 62 & Class 0 & 63 & NIR, FIR, mm, cm & $64,65,66,67$ & Yes & 68 & Yes & 63 \\
\hline & $20049+2721$ & $236^{d}$ & - & - & - & $\mathrm{NIR}, \mathrm{cm}$ & 64,67 & - & - & $?$ & - \\
\hline V1057 Cyg & $20571+4403$ & 200 & 69 & FU Or & 70 & NIR, IR, FIR, cm, mm, smm & $71,72,73,74$ & Yes & 75 & Yes & 76 \\
\hline CB 232 & $21352+4307$ & 14 & 41 & Class I & 42 & $\mathrm{NIR}, \mathrm{mm}, \mathrm{smm}$ & $42,41,77$ & Yes & 58 & Yes & 45 \\
\hline IC $1396 \mathrm{E}$ & $21391+5802$ & 440 & 78 & Class 0 & 78 & NIR, FIR, mm, smm, cm, X-ray & $79,80,78,98,106$ & Yes & 34 & Yes & 40 \\
\hline L1165 & $22051+5848$ & 120 & 81 & Class I/FU Or & 81 & NIR & 81,82 & No & 83 & Yes & 84,85 \\
\hline IRAS 22134 & $22134+5834$ & 7943 & 111 & $-^{a}$ & - & NIR, FIR, mm & $107,86,108$ & No & 110 & Yes & 86 \\
\hline L1221 & $22266+6845$ & 2.7 & 87 & Class I & 102 & $\mathrm{NIR}, \mathrm{mm}$ & 103,99 & No & 88 & Yes & 87 \\
\hline \multirow[t]{3}{*}{ NGC 7538} & IRS $1-3^{b}$ & $\sim 250000$ & 89 & $-^{a}$ & - & NIR, FIR, mm & 89,90 & Yes & 91,92 & Yes & 93 \\
\hline & $\operatorname{IRS} 9^{b}$ & $\sim 60000$ & 89 & $-^{a}$ & - & NIR, FIR, mm, cm, smm & $89,100,101$ & Yes & 92 & Yes & 93 \\
\hline & IRS $11^{b}$ & 10000 & 67 & $-^{a}$ & - & FIR, mm, smm & 89,90 & Yes & 92 & Yes & 93 \\
\hline
\end{tabular}

Notes. ${ }^{(a)}$ Probably young massive star/stars. ${ }^{(b)}$ NIR source. No IRAS source at this position. ${ }^{(c)}$ Submillimeter luminosity. ${ }^{(d)}$ IRAS luminosity.

References. (1) Yang et al. (1990); (2) Han et al. (1998); (3) this work; (4) Anglada et al. (1997); (5) Moorkeja et al. (1999); (6) Fiebig (1997); (7) Carpenter et al. (1993); (8) McCutcheon et al. (1995); (9) McMuldroch et al. (1995); (10) Anglada et al. (1994); (11) Snell et al. (1990); (12) Yang (1990); (13) Wouterloot et al. (1993); (14) Henning et al. (1994); (15) Carpenter et al. (1990); (16) Henning et al. (1992); (17) Gómez et al. 1997; (18) Cohen et al. 1985; (19) Padgett et al. (1999); (20) Moriarty-Schieven et al. (1994); (21) Strom et al. (1986); (22) Chen et al. (1995); (23) Devine et al. (1999); (24) Hoddap \& Ladd (1995); (25) Rodríguez et al. (1995); (26) Reipurth et al. (1993); (27) Beltrán et al. (2002)a; (28) Davis et al. (1997); (29) Dent et al. (1998); (30) Felli et al. (1992); (31) Beuther et al. (2002a); (32) Porras et al. (2000); (33) Yao et al. (2000); (34) Tofani et al. (1995); (35) Zavagno et al. (1997); (36) Chen \& Tokunaga (1994); (37) Price et al. (1983); (38) Morgan et al. (1990); (39) Wouterloot \& Walmsley (1986); (40) Wilking et al. (1990); (41) Launhardt \& Henning (1997); (42) Yun \& Clemens (1995); (43) Launhardt et al. (1997); (44) Yun et al. (1996); (45) Yun \& Clemens (1994)a; (46) Reipurth \& Olberg (1991); (47) Garnavich et al. (1997); (48) Rodríguez et al. (1998); (49) Palla et al. (1993); (50) Reipurth et al. (1996); (52) Cernicharo et al. (1997); (53) Gredel \& Reipurth (1993); (54) Stapelfeldt \& Scoville (1993); (55) Rodríguez \& Reipurth (1994); (56) Yun \& Clemens (1994)b; (57) Moreira et al. (1997); (58) Gómez et al. (2006); (59) Kelly \& Macdonald (1996); (60) Codella et al. (1996); (61) Chini et al. (1997); (62) Gregersen et al. (1997); (63) Bachiller et al. (1995); (64) Chen et al. (1997); (65) Di Francesco et al. (1998); (66) Choi et al. (1999); (67) Anglada et al. (1998a); (68) Brand et al. (1994); (69) Kenyon (1999); (70) Herbig (1977); (71) Greene \& Lada (1977); (72) Kenyon \& Hartmann (1991); (73) Rodríguez \& Hartmann (1992); (74) Weintraub et al. (1991); (75) Rodríguez et al. (1987); (76) Evans II et al. (1994); (77) Huard et al. (1999); (78) Sugitani et al. (2000); (79) Wilking et al. (1993); (80) Saraceno et al. (1996); (81) Reipurth \& Aspin (1997); (82) Tapia et al. (1997); (83) Persi et al. (1994); (84) Parker et al. (1991); (85) Reipurth et al. (1997a); (86) Dobashi et al. (1994); (87) Umemoto et al. (1991); (88) Claussen et al. (1996); (89) Werner et al. (1979); (90) Akabane et al. (1992); (91) Genzel \& Downes (1977); (92) Kameya et al. (1990); (93) Kameya et al. (1989); (94) Minchin \& Murray (1994); (95) Quanz et al. (2007); (96) Leurini et al. (2007); (97) Choi \& Tang (2006); (98) Beltrán et al. (2002)b; (99) Lee \& Ho (2005); (100) Sánchez-Monge et al. (2008); (101) Sandell et al. (2005); (102) Lee et al. (2002); (103) Connelley et al. (2007); (104) Longmore et al. (2006); (105) Ciardi \& Gómez-Martín (2007); (106) Getman et al. (2007); (107) Kumar et al. (2006); (108) Wu et al. (2007); (109) Busquet et al. (2009); (110) Sridharan et al. (2002); (111) Williams et al. (2005).

obtained for their associated high-density cores are smaller than $100 M_{\odot}$. In general, the values derived for the mass coincide with the virial mass within a factor of 3 . This overall trend suggests that most of the observed condensations are near the virial equilibrium and that the assumed $\mathrm{NH}_{3}$ abundance is adequate. However, we found four sources (HH 265, L588, L673, IRAS
$20050+2720)$ for which the derived mass exceeds the virial mass by a large factor $(>5)$. This result could imply that these clouds are still in the process of gravitational collapse.

Recently, Wu et al. (2010) find a strong correlation between the luminosity of a sample of high-mass sources and the mass of the cores, traced by several high-density tracers. Although 


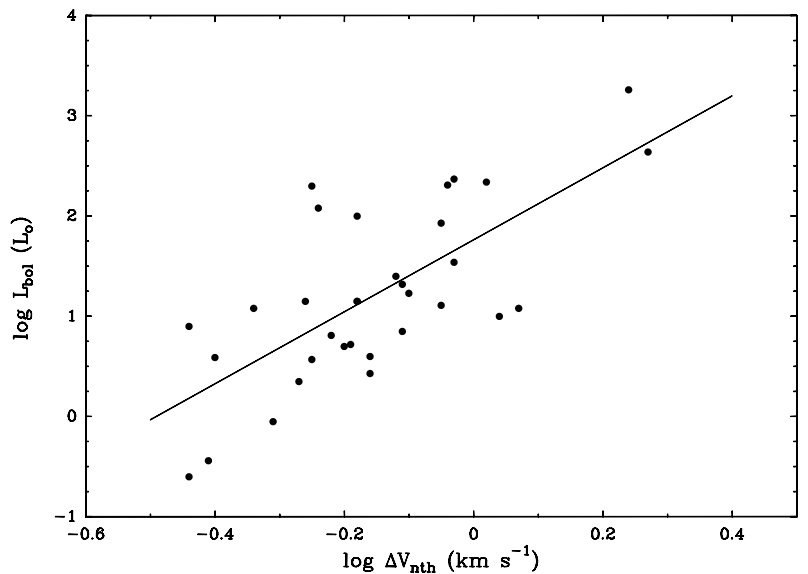

Fig. 4. Bolometric luminosity vs. nonthermal line width for the observed regions with $D \leq 1 \mathrm{kpc}$ (regions observed in Paper I are also included).

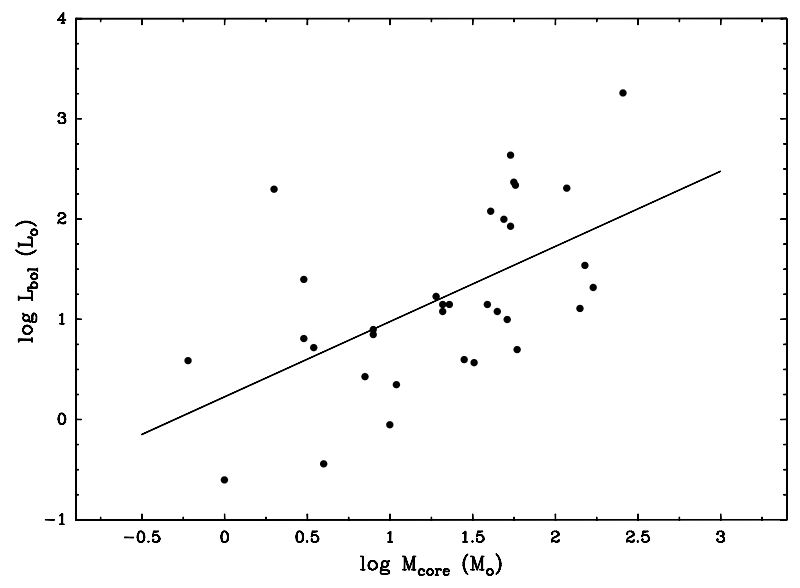

Fig. 5. Diagram of bolometric luminosity vs. mass of the core for the observed regions with $D \leq 1 \mathrm{kpc}$ (regions observed in Paper I are also included).

their sample spans a wide range of distances, they assume that there is no distance effect on the clump masses. In order to check this correlation, and to ensure that there is no distance bias in our sample (see above), we selected sources closer than $1 \mathrm{kpc}$. In Fig. 5 we plot the luminosity of the source as a function of the mass of the core for the sources observed in this paper and in Paper I, using only sources with $D \leq 1 \mathrm{kpc}$. We found that both parameters are related by $\log \left(L_{\mathrm{bol}} / L_{\odot}\right)=$ $(0.8 \pm 0.2) \log \left(M_{\text {core }} / M_{\odot}\right)+(0.2 \pm 0.3)$ with a correlation coefficient of 0.6 . The correlation we found for this sample is lower than the value we would obtain for the complete sample, including the more distant sources. Although for the complete sample the correlation can be caused by the distance bias of the sample (more luminous sources are, in general, more distant), for the sources closer than $1 \mathrm{kpc}$ there is still a significant correlation between luminosity and core mass, and this correlation is not caused by any distance bias. This result indicates that the sources formed in massive clumps can accrete more material and form more massive stars that are also more luminous.

\section{Evolutive differences in the outflow sources}

We detected and mapped the $\mathrm{NH}_{3}$ emission in 24 out of 30 regions associated with molecular outflow in our sample $(80 \%)$. In four of the six regions where we failed in detecting ammonia emission, the evidence for $\mathrm{CO}$ outflow is weak. In the 24 regions associated with molecular outflow, the $\mathrm{NH}_{3}$ emission is usually strong; the ammonia emission is faint $\left(T_{\mathrm{MB}} \leq 0.5 \mathrm{~K}\right.$; see Table 2) only in three regions (IRAS 05490+2658, V1057 Cyg and L1165). On the other hand, in the regions without molecular outflow, the ammonia emission is usually undetectable or very faint. These results agree well with the results we obtained in Paper I, where we studied the relationship between the type of outflow and the intensity of the $\mathrm{NH}_{3}$ emission from a small sample of sources.

In order to substantiate the relationship between the type of outflow and the intensity of the ammonia emission, here we continue the study we began in Paper I, with a more complete sample of regions. The sample includes the regions observed in the present paper, the sources reported in Paper I, and also the results of other Haystack $\mathrm{NH}_{3}$ observations reported in the literature. We studied the distribution of the intensity of the $\mathrm{NH}_{3}$ emission, as measured by the main beam brightness temperature toward the outflow exciting source in this large sample of regions. As the main beam brightness temperature is a good measure of the intensity of the $\mathrm{NH}_{3}$ emission only for sources that fill the beam of the telescope, we included in the sample only the sources whose angular size of the ammonia emission is higher than the telescope beam. Thus, we used only sources with $D \leq 1 \mathrm{kpc}$.

Our final sample is presented in Table 7. It contains a total of 79 sources, with 30 sources associated with only molecular outflow, 40 sources associated with both molecular and optical outflows and 9 sources with only optical outflow.

In Fig. 6 (left) we present the distribution of the $\mathrm{NH}_{3}$ main beam brightness temperature toward the position of the proposed outflow exciting source (Table 7) for the three groups of sources. The mean values of the $\mathrm{NH}_{3}$ brightness temperature are $\left\langle T_{\mathrm{MB}}\right\rangle=$ $0.42 \mathrm{~K}$ for regions with only optical outflow, $\left\langle T_{\mathrm{MB}}\right\rangle=1.35 \mathrm{~K}$ for regions with optical and molecular outflow, and $\left\langle T_{\mathrm{MB}}\right\rangle=1.34 \mathrm{~K}$ for sources with molecular outflow only.

Clearly the sources with only optical outflow tend to present lower values of the $\mathrm{NH}_{3}$ brightness temperature, while the distribution for sources with molecular outflow is shifted to higher values of the brightness temperature. The displacement to higher values of $T_{\mathrm{MB}}$ is similar for sources with only molecular outflow as for sources with both optical and molecular outflow. This was an expected result, because recent studies with high sensitivity detectors are revealing weak $\mathrm{HH}$ objects toward regions of high visual extinction, where previous observations failed in the detection. In our study, we did not take into account differences in the brightness of the Herbig-Haro objects or in the strength of the molecular outflow. We conclude, therefore, that the ammonia emission is in general more intense in molecular outflow sources than in sources without molecular outflow.

A similar result is obtained for the derived ammonia column densities. In Fig. 6 (right) we show the distribution of the derived ammonia column density (Table 7) for the three groups of sources. We note that the distribution for sources with $\mathrm{CO}$ outflow is shifted to higher values of the $\mathrm{NH}_{3}$ column density, while for sources with only $\mathrm{HH}$ outflow the distribution tends to lower values. The mean values for the $\mathrm{NH}_{3}$ column density are $\left\langle N\left(\mathrm{NH}_{3}\right)\right\rangle=5.2 \times 10^{13} \mathrm{~cm}^{-2}$ for regions with optical outflow only, $\left\langle N\left(\mathrm{NH}_{3}\right)\right\rangle=1.94 \times 10^{14} \mathrm{~cm}^{-2}$ for sources with molecular 
A\&A 527, A41 (2011)

Table 7. Regions associated with molecular or optical outflow observed in $\mathrm{NH}_{3}$.

\begin{tabular}{|c|c|c|c|c|c|c|c|}
\hline Source & $\begin{array}{l}\text { Outflow } \\
\text { associated }\end{array}$ & Ref. & $\begin{array}{r}T_{\mathrm{MB}}{ }^{a} \\
(\mathrm{~K})\end{array}$ & $\begin{array}{c}N\left(\mathrm{NH}_{3}\right)^{b} \\
\left(10^{14} \mathrm{~cm}^{-2}\right)\end{array}$ & Ref. & $\begin{array}{c}D \\
(\mathrm{pc})\end{array}$ & Ref \\
\hline M120.1+3.0-N (IRAS 00213+6530) & $\mathrm{CO}$ & 1 & 0.65 & 0.9 & 2 & 850 & 1 \\
\hline M120.1+3.0-S (IRAS 00259+6510) & $\mathrm{CO}$ & 1 & 0.57 & 0.7 & 2 & 850 & 1 \\
\hline L1287 & $\mathrm{CO}$ & 1 & 2.51 & 4.6 & 2 & 850 & 1 \\
\hline L1293 & $\mathrm{CO}$ & 1 & 1.06 & 1.1 & 2 & 850 & 1 \\
\hline L1448 IRS1 & $\mathrm{CO}, \mathrm{HH}$ & 72,70 & 0.3 & 0.3 & 4 & 350 & 3 \\
\hline L1448 IRS2 & $\mathrm{CO}, \mathrm{HH}$ & 70 & 1.9 & 1.8 & 4 & 350 & 3 \\
\hline L1448 IRS3 & $\mathrm{CO}, \mathrm{HH}$ & 23,5 & 3.1 & 4.2 & 4 & 350 & 3 \\
\hline L1448 C & $\mathrm{CO}, \mathrm{HH}$ & 23,70 & 2.5 & 3.3 & 4 & 350 & 3 \\
\hline GL490 & $\mathrm{CO}$ & 6 & $\leq 0.5$ & $\leq 0.6$ & 7 & 900 & 8 \\
\hline L1455 IRS1 & $\mathrm{CO}, \mathrm{HH}$ & 9,70 & 1.9 & 1.0 & 4 & 350 & 3 \\
\hline L1455 IRS2 & $\mathrm{CO}, \mathrm{HH}$ & 9,70 & 2.5 & 1.6 & 4 & 350 & 3 \\
\hline L1489 & $\mathrm{CO}, \mathrm{HH}$ & 10,71 & 0.8 & 1.8 & 11 & 140 & 8 \\
\hline НH 156 & $\mathrm{HH}$ & 1 & $\leq 0.2$ & $\leq 0.4$ & 2 & 140 & 1 \\
\hline HH 159 & $\mathrm{CO}, \mathrm{HH}$ & 1 & $\leq 0.4$ & $\leq 0.8$ & 2 & 160 & 1 \\
\hline HH 158 & $\mathrm{HH}$ & 1 & $\leq 0.3$ & $\leq 0.6$ & 2 & 160 & 1 \\
\hline HH 31 & $\mathrm{CO} ?, \mathrm{HH}$ & 1 & 0.56 & 0.7 & 2 & 160 & 1 \\
\hline L1524 (Haro 6-10) & $\mathrm{CO}, \mathrm{HH}$ & 73,14 & $\leq 0.6$ & $\leq 1$ & 4 & 140 & 13 \\
\hline L1551 IRS 5 & $\mathrm{CO}, \mathrm{HH}$ & 15,16 & 2.72 & 2.3 & 7 & 140 & 13 \\
\hline HL Tau & $\mathrm{CO}, \mathrm{HH}$ & 17,18 & $\leq 1$ & $\leq 1$ & 7 & 140 & 13 \\
\hline L1551 NE & $\mathrm{CO}, \mathrm{HH}$ & 1 & 0.79 & 1.0 & 2 & 160 & 1 \\
\hline L1642 & $\mathrm{CO}, \mathrm{HH}$ & 1 & $\leq 0.1$ & $\leq 0.1$ & 2 & 125 & 1 \\
\hline L1527 & $\mathrm{CO}, \mathrm{HH}$ & $20,74,19$ & 2.12 & 5.0 & 11 & 140 & 13 \\
\hline L1634 (IRAS 05173-0555) & $\mathrm{CO}, \mathrm{HH}$ & 1 & 1.0 & 1.1 & 2 & 460 & 1 \\
\hline L1634 (IRS 7) & $\mathrm{CO}, \mathrm{HH}$ & 83,84 & 0.59 & 0.8 & 2 & 460 & 1 \\
\hline RNO 43 (IRAS 05295+1247) & $\mathrm{CO}, \mathrm{HH}$ & 21,22 & 0.40 & 0.4 & 2 & 400 & 24 \\
\hline $\mathrm{HH} 83$ & $\mathrm{CO}, \mathrm{HH}$ & 26,25 & 0.46 & $0.4-0.5$ & 85 & 470 & 13 \\
\hline HH 84 & $\mathrm{HH}$ & 25 & 0.3 & $0.5-1$ & 85 & 470 & 13 \\
\hline HH $33 / 40$ & $\mathrm{HH}$ & 27 & $\leq 0.3$ & $\leq 0.5$ & 28 & 470 & 13 \\
\hline НН 86/87/88 & $\mathrm{HH}$ & 25 & $\leq 0.2$ & $\leq 0.1$ & 85 & 470 & 13 \\
\hline HH 34 & $\mathrm{CO}, \mathrm{HH}$ & 30,29 & 1.3 & 1.1 & 4 & 480 & 31 \\
\hline L1641-N & $\mathrm{CO}, \mathrm{HH}$ & 32,69 & 2.2 & 2.8 & 85 & 480 & 33 \\
\hline HH $38-43$ & $\mathrm{HH}$ & 27 & $\leq 0.5$ & $\leq 0.5$ & 4 & 480 & 31 \\
\hline Haro 4-255 FIR & $\mathrm{CO}$ & 72 & 2.2 & 2.1 & 4 & 480 & 31 \\
\hline L1641-S3(high velocity) & $\mathrm{CO}$ & 1 & 2.0 & 2.4 & 2 & 480 & 1 \\
\hline HH 68 & $\mathrm{HH}$ & 1 & $\leq 0.3$ & $\leq 0.4$ & 2 & 460 & 1 \\
\hline B35 & $\mathrm{CO}$ & 10 & 1.2 & 4.0 & 11 & 500 & 34 \\
\hline HH 26 IR & $\mathrm{CO}, \mathrm{HH}$ & 36,35 & 2.8 & 2.5 & 7 & 470 & 13 \\
\hline HH 25 MMS & $\mathrm{CO}, \mathrm{HH}$ & 75,35 & 2.4 & 2.1 & 7 & 470 & 13 \\
\hline NGC 2071 & $\mathrm{CO}$ & 37 & 2.44 & 2.5 & 7 & 500 & 8 \\
\hline HH 270 IRS & $\mathrm{HH}$ & 1 & 0.67 & 0.7 & 2 & 460 & 1 \\
\hline IRAS $05487+0255$ & $\mathrm{CO}$ & 1 & 0.8 & 0.7 & 2 & 460 & 1 \\
\hline HH 111 & $\mathrm{CO}, \mathrm{HH}$ & 1 & 0.56 & 0.5 & 2 & 460 & 1 \\
\hline Mon R2 & $\mathrm{CO}$ & 38 & 1.2 & 1.2 & 7 & 800 & 8 \\
\hline Mon R2-N & $\mathrm{CO}$ & 76 & 1.0 & 1.0 & 7 & 800 & 8 \\
\hline GGD 12-15 & $\mathrm{CO}$ & 39 & 1.52 & 2.0 & 7 & 1000 & 8 \\
\hline RMon & $\mathrm{CO}, \mathrm{HH}$ & 40,35 & $\leq 0.5$ & $\leq 0.6$ & 7 & 800 & 13 \\
\hline NGC 2264 (HH 14-4/5/6) & $\mathrm{HH}$ & 41 & $\leq 1$ & $\leq 1$ & 4 & 800 & 13 \\
\hline HH 120 & $\mathrm{CO}, \mathrm{HH}$ & $43,77,42$ & 1.8 & 2.5 & 44 & 400 & 45 \\
\hline L1709 & $\mathrm{CO}$ & 1 & $\leq 0.4$ & $\leq 0.6$ & 2 & 160 & 1 \\
\hline L43 & $\mathrm{CO}$ & 72 & 2.7 & 2.2 & 4 & 160 & 46 \\
\hline L100 & $\mathrm{CO}$ & 47 & 0.5 & $0.3-0.5$ & 85 & 225 & 48 \\
\hline L483 & $\mathrm{CO}$ & 47 & 4.54 & 14 & 85 & 200 & 49 \\
\hline L588 & $\mathrm{CO}, \mathrm{HH}$ & 1 & 1.3 & 4.1 & 2 & 310 & 1 \\
\hline R CrA (HH 100-IR) & $\mathrm{CO}, \mathrm{HH}$ & $72,50,79$ & 2.8 & 2.0 & 4 & 130 & 51 \\
\hline $\mathrm{R} \mathrm{CrA} \mathrm{(IRS} \mathrm{7)}$ & $\mathrm{CO}, \mathrm{HH}$ & $72,50,78$ & 1.6 & 1.8 & 4 & 130 & 51 \\
\hline L673 & $\mathrm{CO}$ & 52 & 2.1 & $\geq 2.2$ & 2,85 & 300 & 3 \\
\hline CB 188 & $\mathrm{CO}$ & 1 & $\leq 0.2$ & $\leq 0.4$ & 2 & 300 & 1 \\
\hline HH 32a & $\mathrm{CO}, \mathrm{HH}$ & 17,35 & $\leq 0.6$ & $\leq 0.7$ & 4 & 300 & 13 \\
\hline L778 & $\mathrm{CO}$ & 10 & 1.8 & 6.3 & 11 & 250 & 8 \\
\hline B335 & $\mathrm{CO}, \mathrm{HH}$ & 53,54 & 1.2 & 7.9 & 11 & 250 & 13 \\
\hline L797 & $\mathrm{CO}$ & 1 & $\leq 0.2$ & $\leq 0.4$ & 2 & 700 & 1 \\
\hline IRAS 20050 & $\mathrm{CO}$ & 1 & 1.7 & 3.6 & 2 & 700 & 1 \\
\hline V1057 Cyg & $\mathrm{CO}$ & 1 & 0.3 & 0.2 & 2 & 700 & 1 \\
\hline L1228 & $\mathrm{CO}, \mathrm{HH}$ & 55,56 & 2.71 & 5.3 & 85 & 300 & 56 \\
\hline V1331 Cyg & $\mathrm{CO}, \mathrm{HH}$ & 72,80 & 0.5 & 2.3 & 4 & 700 & 57 \\
\hline
\end{tabular}


Table 7. continued.

\begin{tabular}{lllrcrcc}
\hline \hline Source & $\begin{array}{l}\text { Outflow } \\
\text { associated }\end{array}$ & Ref. & $\begin{array}{r}T_{\mathrm{MB}}{ }^{a} \\
(\mathrm{~K})\end{array}$ & $\begin{array}{c}N\left(\mathrm{NH}_{3}\right)^{b} \\
\left(10^{14} \mathrm{~cm}^{-2}\right)\end{array}$ & Ref. & $\begin{array}{c}D \\
(\mathrm{pc})\end{array}$ & Ref. \\
\hline L1172 & CO & 10 & 1.8 & 7.9 & 11 & 440 & 8 \\
CB 232 & CO & 1 & 0.58 & 0.9 & 2 & 600 & 1 \\
IC 1396 E & CO & 1 & 0.86 & 1.4 & 2 & 750 & 1 \\
NGC 7129 & CO, HH & 58,59 & 0.52 & 0.36 & 7 & 1000 & 13 \\
HHL73 (IRAS 21429+4729) & CO & 60 & 1.66 & 3.2 & 85 & 900 & 61 \\
HHL73 (IRAS 21432+4719) & CO, HHL, HH & $60,61,81$ & 1.25 & 1.1 & 28 & 900 & 61 \\
HHL73 (IRAS 21441+4722) & CO & 60 & 0.7 & $\geq 0.9$ & 85 & 900 & 61 \\
L1165 & CO, HH & 1 & 0.35 & 0.9 & 2 & 750 & 1 \\
S140N (IRAS 22178+6317) & CO & 32 & 0.88 & 1.4 & 28 & 900 & 63 \\
S140N (Star 2) & CO, HH & 82,62 & 1.2 & 1.9 & 28 & 900 & 63 \\
L1221 & CO, HH & 1 & 2.5 & 3.9 & 2 & 200 & 1 \\
L1251 (IRAS 22343+7501) & CO, HH & 64,65 & 0.29 & 1.7 & 85 & 300 & 66 \\
L1251 (IRAS 22376+7455) & CO, HH & 64,67 & 1.71 & 3.1 & 85 & 300 & 66 \\
L1262 & CO & 47 & 1.57 & $\geq 4.9$ & 85 & 200 & 68 \\
\hline
\end{tabular}

Notes. Regions with distance $\leq 1 \mathrm{kpc} .{ }^{a}$ Main brightness temperature at the position of the suspected exciting source. ${ }^{b}$ Lower limit of the beamaveraged column density at the position of the suspected exciting source.

References. (1) see Table 1; (2) this paper; (3) Herbig \& Jones (1983); (4) Anglada et al. (1989); (5) Eiroa et al. (1994a); (6) Snell et al. (1984); (7) Torrelles et al. (1983); (8) Fukui et al. (1993); (9) Goldsmith et al. (1984); (10) Myers et al. (1988); (11) Benson \& Myers (1989); (12) Strom et al. (1986); (13) Reipurth (1994); (14) Elias (1978); (15) Snell et al. (1980); (16) Mundt \& Fried (1983); (17) Edwards \& Snell (1982); (18) Mundt et al. (1988); (19) Eiroa et al. (1994a); (20) Heyer et al. (1987); (21) Edwards \& Snell (1984); (22) Jones et al. (1984); (23) Bachiller et al. (1990); (24) Maddalena \& Morris (1987); (25) Reipurth (1989); (26) Bally et al. (1994); (27) Haro (1953); (28) Verdes-Montenegro et al. (1989); (29) Haro (1959); (30) Chernin \& Masson (1995); (31) Genzel et al. (1981); (32) Fukui et al. (1986); (33) Chen et al. (1993); (34) Felli et al. (1992); (35) Herbig (1974); (36) Snell \& Edwards (1982); (37) Bally (1982); (38) Loren (1981); (39) Rodríguez et al. (1982); (40) Cantó et al. (1981); (41) Adams et al. (1979); (42) Cohen \& Schwartz (1987); (43) Olberg et al. (1989); (44) Persi et al. (1994); (45) Petterson (1984); (46) Chini (1981); (47) Parker et al. (1988); (48) Reipurth \& Gee (1986); (49) Ladd et al. (1991a); (50) Strom et al. (1974); (51) Marraco \& Rydgren (1981); (52) Armstrong \& Winnewisser (1989); (53) Frerking \& Langer (1982); (54) Vrba et al. (1986); (55) Haikala \& Laureijs (1989); (56) Bally et al. (1995); (57) Chavarría-K (1981); (58) Loren (1977); (59) Ray et al. (1990); (60) Dobashi et al. (1993); (61) Gyulbudaghian et al. (1987); (62) Eiroa et al. (1993); (63) Crampton \& Fisher (1974); (64) Sato \& Fukui (1989); (65) Balázs et al. (1992); (66) Kun \& Prusti (1993); (67) Eiroa et al. (1994b); (68) Parker et al. (1991); (69) Reipurth et al. (1998); (70) Bally et al. (1997); (71) Gómez et al. (1997); (72) Levreault (1985); (73) Hogerheijde et al. (1998); (74) Tamura et al. (1996); (75) Gibb \& Davis (1998); (76) Tafalla et al. (1997); (77) Nielsen et al. (1998); (78) Anderson et al. (1997); (79) Whittet et al. (1996); (80) Mundt \& Eislöffel (1998); (81) Devine, Reipurth \& Bally (1997); (82) Davis et al. (1998); (83) Davis et al. (1997); (84) Hoddap \& Ladd (1995); (85) Anglada et al. (1997).

and optical outflow and $\left\langle N\left(\mathrm{NH}_{3}\right)\right\rangle=2.49 \times 10^{14} \mathrm{~cm}^{-2}$ for sources with molecular outflow only.

Recently, Davis et al. (2010) find from a study of outflows and their exciting sources in the Taurus region that sources driving $\mathrm{CO}$ outflows have redder near-IR colours than sources driving $\mathrm{HH}$ jets, and they conclude that $\mathrm{CO}$ outflow sources are more embedded in the high-density gas than the $\mathrm{HH}$ optical outflow sources. This result agrees well with ours.

All these results can be interpreted as an indication that molecular outflow sources are younger, since they are associated with a larger amount of high-density gas. As the star evolves, the surrounding material becomes less dense, decreasing the ammonia column density, and at the same time making the Herbig-Haro objects detectable. At the time when the molecular outflow has disrupted and swept out the molecular material surrounding the $\mathrm{YSO}$, both the $\mathrm{CO}$ outflow and the $\mathrm{NH}_{3}$ column density are expected to be weak, and only the Herbig-Haro objects would be observable.

The ammonia emission and the observational appearance of outflows trace an evolutive sequence of sources. Molecular and optical outflow would be phenomena that dominate observationally at different stages of the YSO evolution. In younger objects, molecular outflows will be prominent, while optical outflows will progressively show up as the YSO evolves.

\section{Conclusions}

We detected the ammonia emission in 27 sources of a sample of 40 sources associated with molecular and/or optical outflows, and we were able to map 25 of them. We also searched for $\mathrm{H}_{2} \mathrm{O}$ maser emission toward 6 sources, and detected new $\mathrm{H}_{2} \mathrm{O}$ masers in HH 265 and AFGL 5173. Our main conclusions can be summarized as follows:

1. In all molecular outflow regions mapped, the $\mathrm{NH}_{3}$ emission peak falls very close to the position of a very good candidate for the outflow excitation (except in the case of IRAS $05490+2658$ where we propose an alternative location for the exciting source). On the other hand, the sources associated with optical outflow (except HH 270 IRS and HH 290 IRS) are not associated with an ammonia emission peak.

2. Four regions (HH 265, L588, L673 and IRAS 20050+2720) could be in the process of gravitational collapse at scales $\geq 0.2 \mathrm{pc}$, as their derived masses exceed the virial mass by a factor $>5$. The rest of ammonia condensations appear to be close to the virial equilibrium.

3. In several regions the ammonia structure presents more than one clump. In the M120.1+3.0-N and IRAS 20050+2720 regions, two clumps with different velocities, which are gravitationally bound though were identified. 

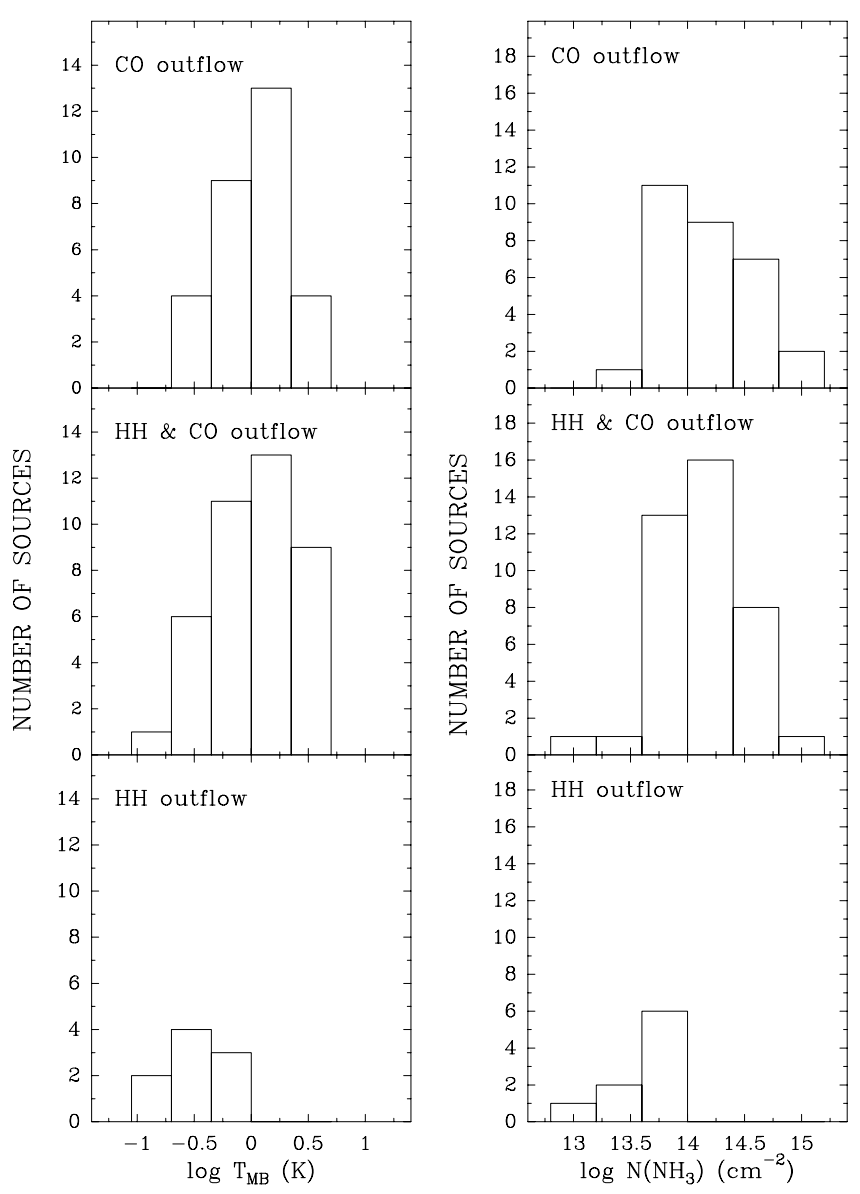

Fig. 6. Distribution of the $\mathrm{NH}_{3}$ main beam brightness temperature (left) and the $\mathrm{NH}_{3}$ column density (right) for sources with only molecular outflow (top), sources with both molecular and optical outflow (middle), and sources with only optical outflow (bottom).

4. We identified a high-density clump where the HH 270/110 jet can suffer the collision responsible for the deflection of the jet.

5. We were able to separate the $\mathrm{NH}_{3}$ emission from the L1641S3 region into two overlapping clouds, one with signs of strong perturbation, probably associated with the driving source of the CO outflow, and a second, quiescent clump, which probably is not associated with star formation.

6. In general, the observed $\mathrm{NH}_{3}$ condensations are very cold, with line widths dominated by nonthermal (turbulent) motions. Among the observed sources, the more massive regions appear to produce a larger perturbation in their molecular high density environment.

7. We found that generally the more luminous objects are associated with broader ammonia lines. A correlation between the nonthermal component of the line width and the luminosity of the associated object, $\log \left(L_{\text {bol }} / L_{\odot}\right)=(3.6 \pm$ $0.9) \log \left(\Delta V_{\text {nth }} / \mathrm{km} \mathrm{s}^{-1}\right)+(1.8 \pm 0.2)$ was found for sources with $D \leq 1 \mathrm{kpc}$.

8. We found that there is a significant correlation between the luminosity of the source and the mass of the core and that this correlation is not caused by any distance bias in the sample. Both parameters are related by $\log \left(L_{\mathrm{bol}} / L_{\odot}\right)=$ $(0.8 \pm 0.2) \log \left(M_{\text {core }} / M_{\odot}\right)+(0.2 \pm 0.3)$.
9. The ammonia brightness temperature and column density of the sources decrease as the outflow activity becomes prominent in the optical. These results give an evolutive scheme in which young objects progressively lose their surrounding high-density gas. The ammonia emission and the observational appearance of outflows trace an evolutive sequence of sources.

Acknowledgements. I.S. acknowledges the Instituto de Astrofísica de Andalucía for the hospitality during part of the preparation of this paper. G.A., R.E., R.L. and J.M.G. acknowledge support from MCYT grant AYA2008-06189-C03 (including FEDER funds). G.A. acknowledges support from Junta de Andalucía, Spain.

\section{References}

Afonso, J. M., Yun, Y. L., \& Clemens, D. P. 1998, AJ, 115, 1111 Akabane, K., Tsunekawa, S., Inoue, M., et al. 1992, PASJ, 44, 421 Alten, V. P., Bally, J., Devine, D., \& Miller, G. J. 1997, in Low Mass Star Formation - from Infall to Outflow, ed. F. Malbet, \& A. Castets, poster, Proc. IAU Symp., 182, 51

Alves, J. F. 1995, Master Thesis, University of Lisbon

Alves, J. F., \& Yun, J. L. 1995, ApJ, 438, L107

Alves, J. F., Hartmann, L., Briceño, C., \& Lada, C. J. 1997, AJ, 113, 1395

Anderson, I. M., Harju, J., Knee, L. B. G., \& Haikala, L. K. 1997, A\&A, 321, 575

André, Ph., Ward-Thompson, D., \& Barsony, M. 1993, ApJ, 406, 122

André, Ph., Ward-Thompson, D., \& Barsony, M. 2000, in Protostars and Planets

IV, ed. V. Mannings, A. P. Boss, \& S. S. Russell (Tucson: Univ. of Arizona Press), 59

Anglada, G., Rodríguez, L. F., Torrelles, J. M., et al. 1989, ApJ, 341, 208

Anglada, G., Rodríguez, L. F., Girart, J. M., Estalella, R., \& Torrelles, J. M. 1994, ApJ, 420, 91

Anglada, G., Estalella, R., Mauesberger, R., et al. 1995, ApJ, 443, 682

Anglada, G., Sepúlveda, I., \& Gómez, J. F. 1997, A\&AS, 121, 255

Anglada, G., Rodríguez, L. F., \& Torrelles, J. M. 1998a, ASPC, 132, 303

Anglada, G., Villuendas, E., Estalella, R., et al. 1998b, AJ, 116, 2953

Arce, H. G., \& Sargent, A. I. 2006, ApJ, 646, 1070

Armstrong, J. T., \& Winnewisser, G. 1989, A\&A, 210, 373

Bachiller, R., Fuente, A., \& Tafalla, M. 1995, ApJ, 445, L51

Bally, J., Devine, D., \& Alten, V. 1997, ApJ, 478, 603

Beltrán, M. T., Estalella, R., Ho, P. T. P., et al. 2002a, ApJ, 565, 1069

Beltrán, M. T., Girart, J. M., Estalella, R., Ho, P. T. P., \& Palau, A. 2002b, ApJ, 573, 246

Beltrán, M. T., Estalella, R., Girart, J. M., Ho, P. T. P., \& Anglada, G. 2008, A\&A, 481, 93

Beuther, H., Schilke, P., Gueth, F., et al. 2002a, A\&A, 387, 931

Beuther, H., Schilke, P., Menten, K. M., et al. 2002b, ApJ, 566, 945

Beuther, H., Leurini, S., Schilke, P., et al. 2007, A\&A, 466, 1065

Benson, P. J., \& Myers, P. C. 1989, ApJS, 71, 89

Blitz, L., Fich, M., \& Stark, A. A. 1982, ApJS, 49, 183

Brand, J., Cesaroni, R., Caselli, P., et al. 1994, A\&AS, 103, 541

Busquet, G., Palau, A., Estalella, R., et al. 2009, A\&A, 506, 1183

Campbell, B., \& Thompson, R. I. 1984, ApJ, 279, 650

Cao, Y. X., Zeng, Q., Deguchi, S., Kameya, O., \& Kaifu, N. 1993, AJ, 105, 1027

Carpenter, J. M., Snell, R. L., \& Schloerb, F. P. 1990, ApJ, 362, 147

Carpenter, J. M., Snell, R. L., Schloerb, F. P., \& Skrutskie, M. F. 1993, ApJ, 407, 657

Carpenter, J. M., Snell, R. L., \& Schloerb, F. P. 1995, ApJ, 450, 201

Cesaroni, R., Walmsley, C. M., Koempe, C., \& Churchwell, E. 1991, A\&A, 252, 278

Cesaroni, R., Felli, M., \& Walmsley, C. M. 1999, A\&AS, 136, 333

Cernicharo, J., Neri, R., \& Reipurth, B. 1997, in Herbig-Haro Flows and the

Birth of Low Mass Stars, ed. B. Reipurth, \& C. Bertout, IAU Symp., 182, 141

Chen, H., \& Tokunaga, A. T. 1994, ApJS, 90, 149

Chen, H., Myers, P. C., Ladd, E. F., \& Wood, D. O. S. 1995, ApJ, 455, 377

Chen, H., Tafalla, M., Greene, T. P., Myers, P. C., \& Wilner, D. J. 1997, ApJ, 475,163

Chini, R., Reipurth, B., Sievers, A., et al. 1997, A\&A, 325, 542

Choi, M., \& Tang, Y.-W. 2006, ApJ, 648, 504

Choi, M., Panis, J.-F., Evans, N. J. II 1999, ApJS, 122, 519

Ciardi, D. R., \& Gómez-Martín, C. 2007, ApJ, 664, 377 
Claussen, M. J., Wilking, B. A., Benson, P. J., et al. 1996, ApJS, 106, 111

Clemens, D. P., \& Barvainis, R. 1988, ApJS, 68, 257

Codella, C., \& Scappini, F. 1998, MNRAS, 298, 1092

Codella, C., Felli, M., \& Natale, V. 1996, A\&A, 311, 971

Cohen, M. 1980, AJ, 85, 29

Cohen, M. \& Schwartz, R. D. 1983, ApJ, 265, 877

Cohen, M., Harvey, P. M., \& Schwartz, R. D. 1985, ApJ, 296, 633

Connelley, M. S., Reipurth, B., \& Tokunaga, A. T. 2007, AJ, 133, 1528

Davis, C. J., Mundt, R., \& Eislöffel, J. 1994, ApJ, 437, L55

Davis, C. J., Ray, T. P., Eislöffel, J., \& Corcoran, D. 1997, A\&A, 324, 263

Davis, C. J., Moriarty-Schieven, G., Eislöffel, J., Hoare, M. G., \& Ray, T. P. 1998, AJ, 115, 1118

Davis, C. J., Chrysostomou, A., Hatchell, J., et al. 2010, MNRAS, in press

Dent, W. R. F., Matthews, H. E., \& Ward-Thompson, D. 1998, MNRAS, 301, 1049

Devine, D., Reipurth, B., \& Bally, J. 1997, in Low Mass Star Formation - from Infall to Outflow, ed. F. Malbet, \& A. Castets, poster, Proc. IAU Symp., 182, 91

Devine, D., Reipurth, B., \& Bally, J. 1999, AJ, 118, 972

Di Francesco, J., Evans II, N. J., Harvey, P. M., Mundy, L. G., \& Butner, H. M. 1998, ApJ, 509, 324

Dobashi, K., \& Uehara, H. 2001, PASJ, 53, 799

Dobashi, K., Nozawa, S., Hayashi, Y., Sato, F., \& Fukui, Y. 1994, AJ, 107, 2148

Emerson, J. P. 1987, in Star Forming Regions, IAU Symp., 115, 19

Estalella, R., Mausberger, R., Torrelles, J. M., et al. 1993, ApJ, 419, 698

Evans, II, N. J., Balkum, S., Levreault, R. M., Hartmann, L., \& Kenyon, S. 1994 ApJ, 424, 793

Felli, M., Palagi, F. \& Tofani, G. 1992, A\&A, 255, 293

Fiebig, D. 1995, A\&A, 298, 207

Fiebig, D. 1997, A\&A, 327, 758

Fiebig, D., Duschl, W. J., Menten, K. M., \& Tscharnuter, W. M. 1996, A\&A, 310, 199

Fukui, Y. 1989, in Low Mass Star Formation and Pre-Main Sequence Objects, ed. B. Reipurth (Garching: ESO), 95

Garnavich, P. M., Noriega-Crespo, A., \& Green, P. J. 1992, Rev. Mex. Astron. Astrofis., 24, 99

Garnavich, P. M., Noriega-Crespo, A., Raga, A. C., \& Bohm, K.-H. 1997, ApJ, 490, 752

Genzel, R., \& Downes, D. 1977, A\&AS, 30, 145

Getman, K. V., Feigelson, E. D., Garmire, G., Bross, P., \& Wang, J. 2007, ApJ, 654,316

Gibb, A. G., \& Davis, C. J. 1998, AJ, 115, 1554

Gredel, R., \& Reipurth, B. 1993, ApJ, 407, L29

Greene, T. P., \& Lada, C. J. 1997, AJ, 114, 2517

Gregersen, E. M., Evans, N. J. II, Zhou, S., \& Choi, M. 1997, ApJ, 484, 256

Gómez, J. F., Gregorio-Monsalvo, I., Súarez, O., \& Kuiper, T. B. H. 2006, AJ, 132,1322

Gómez, M., Whitney, B. A., \& Kenyon, S. J. 1997, AJ, 114, 1138

Gyulbudaghian, A. L. 1982, PAZh, 8, 232

Han, F., Mao, R. Q., Lu, J., et al. 1998, A\&AS, 127, 181

Harju, J., Walmsley, C. M., \& Wouterloot, J. G. A. 1993, A\&AS, 98, 51

Hayashi, M., Hasegawa, T., Ohashi, N., \& Kazuyoshi, S. K. 1994, ApJ, 426, 234

Henning, Th., Cesaroni, R., Walmsley, M., \& Pfau, W. 1992, A\&AS, 93, 525

Henning, Th., Martin, K., Reimann, H.-G., et al. 1994, A\&A, 288, 282

Herbig, G. H. 1974, Lick. Obs. Bull., No. 658

Herbig, G. H. 1977, ApJ, 217, 693

Herbig, G. H., \& Jones, B. F. 1983, AJ, 88, 1040

Hilton, J., White, G. J., Rainey, R., \& Cronin, N. J. 1986, A\&A, 154, 274

Ho, P. T. P., \& Townes, C. H. 1983, ARA\&A, 21, 231

Hoddap, K.-W., \& Ladd, E. F. 1995, ApJ, 453, 715

Hogerheijde, M. R., van Dishoeck, E. F., Blake, G. A., \& van Langevelde, H. J. 1998, ApJ, 502, 315

Huard, T. L., Sandell, G., \& Weintraub, D. A. 1999, ApJ, 526, 833

Hughes, V. A., \& MacLeod, G. C. 1994, ApJ, 427, 857

Jones, B. F., Cohen, M., Sirk, M., \& Jarrett, R. 1984, AJ, 89, 1404

Kameya, O., Hasegawa. T. I., Hirano, N., et al. 1986, PASJ, 38, 793

Kameya, O., Hasegawa, T. I., Hirano, N., Takakubo, K., \& Seki, M. 1989, ApJ, 339,222

Kameya, O., Morita, K.-I., Kawabe, R., \& Ishiguro, M. 1990, ApJ, 355, 562

Kelly, M. L., \& Macdonald, G. H. 1995, Ap\&SS, 224, 497

Kelly, M. L., \& Macdonald, G. H. 1996, MNRAS, 282, 401

Kenyon, S. J. 1999, in The Origin of Stars and Planetary Systems, ed. C. J. Lada, \& N. D. Kylafis (Kluwer Academic Publishers), 613

Kenyon, S. J., \& Hartmann, L. W. 1991, ApJ, 383, 664

Kenyon, S. J., Hartmann, L., Gómez, M., Carr, J. S., \& Tokunaga, A. 1993, AJ, 105,1505

Kumar, M. S. N., Bachiller, R., \& Davis, C. J. 2002, ApJ, 576, 313

Kumar, M. S. N., Ojha, D. K., \& Davis, C. J. 2003, ApJ, 598, 1107
Kumar, M. S. N., Keto, E., \& Clerkin, E. 2006, A\&A, 449, 1033

Kun, M., \& Prusti, T. 1993, A\&A, 272, 235

Lada, C. J. 1991, in Star Forming Regions, ed. M. Peimbert, \& J. Jugaku (Dordrecht: Reidel), IAU Symp., 115, 1

Launhardt, R., \& Henning, Th. 1997, A\&A, 326, 329

Launhardt, R., Ward-Thompson, D., \& Henning, Th. 1997, MNRAS, 288, L45

Launhardt, R., Evans II, N. J., Wang, Y., et al. 1998, ApJS, 119, 59

Lee, C.-F., \& Ho, P. T. P. 2005, ApJ, 632, 964

Lee, C.-F., Mundy, L. G., Reipurth, B., Ostriker, E. C., \& Stone, J. M. 2000, ApJ, 542,925

Lee, C.-F., Mundy, L. G., Stone, J. M., \& Ostriker, E. C. 2002, ApJ, 576, 294

Leurini, S., Beuther, H., Schilke, P., et al. 2007, A\&A, 475, 925

Levreault, R. M. 1988, ApJS, 67, 283

Liljeström, T. 1991, A\&A, 244, 483

Liljeström, T., Mattila, K., \& Friberg, P. 1989, A\&A, 210, 337

Longmore, S. N., Burton, M. G., Minier, V., \& Walsh, A. J. 2006, MNRAS, 369 , 1196

López, R., Estalella, R., Raga, A. C., et al. 2005, A\&A, 432, 567

McCutcheon, W. H., Sato, T., Purton, C. R., Matthews, H. E., \& Dewdney, P. E. 1995, AJ, 110, 1762

McMuldroch, S., Blake, G. A., \& Sargent, A. I. 1995, AJ, 110, 354

Minchin, N. R., \& Murray, A. G., 1994, A\&A, 286, 579

Mitchell, G. F., Hasewaga, T. I., Dent, W. R. F., \& Matthews, H. E. 1994, ApJ, 436, L177

Morata, O., Estalella, R., López, R., \& Planesas, P. 1997, MNRAS, 292, 120

Morata, O., Girart, J. M., \& Estalella, R. 2003, A\&A, 397, 181

Morata, O., Girart, J. M., \& Estalella, R. 2005, A\&A, 435, 113

Moreira, M. C., \& Yun, J. L. 1995, ApJ, 454, 850

Moreira, M. C., Yun, J. L., Vázquez, R., \& Torrelles, J. M. 1997, AJ, 113, 137

Morgan, J. A., Snell, R. L., \& Strom, K. M. 1990, ApJ, 362, 274

Morgan, J. A., Schloerb, F. P., Snell, R. L., \& Bally, J. 1991, ApJ, 376, 618

Moriarty-Schieven, G. H., Wannier, P. G., Tamura, M., \& Keene, J. 1992, ApJ, 400, 260

Moriarty-Schieven, G. H., Wannier, P. G., Keene, J., \& Tamura, M. 1994, ApJ, 436, 800

Moriarty-Schieven, G. H., Butner, H. M., \& Wannier, P. G. 1995, ApJ, 455, L55

Moriarty-Schieven, G. H., Johnstone, D., Bally, J., \& Jenness, T. 2006, ApJ, 645, 357

Movsessian, T. A., Magakian, T. Y., Bally, J., et al. 2007, A\&A, 470, 605

Mundt, R., \& Eislöffel, J. 1998, AJ, 116, 860

Mundt, R., Brugel, E. W., \& Bührke, T. 1987, ApJ, 319, 275

Mundt, R., Ray, T. P., \& Raga, A. C. 1991, A\&A, 252, 740

Nielsen, A. S., Olberg, M., Knude, J., \& Booth, R. S. 1998, A\&A, 336, 329

Ohashi, N., Kawabe, R., Ishiguro, M., \& Hayashi, M. 1991, AJ, 102, 2054

Ohashi, N., Hayashi, M., Kawabe, R., \& Ishiguro, M. 1996, ApJ, 466, 317

Onishi, T., Mizuno, A., Kawamura, A., Ogawa H., \& Fukui, Y. 1998, ApJ, 502, 296

Padgett, D. L., Brandner, W., Stapelfeld, K. R., et al. 1999, AJ, 117, 1490

Palla, F., Cesaroni, R., Brand, J., et al. 1993, A\&A, 280, 599

Parker, N. D., Padman, R., \& Scott, P. F. 1991, MNRAS, 252, 442

Patel, N. A., Greenhill, L. J., Herrnstein, J., et al. 2000, ApJ, 538, 268

Pauls, T. A., Wilson, T. L., Bieging, J. H., \& Martin, R. N. 1983, A\&A, 124, 23

Persi, P., Palagi, F., \& Felli, M. 1994, A\&A, 291, 577

Price, S. D., Murdock, T. L., Shivanandan, K., \& Bowers, P. F. 1983, ApJ, 275, 125

Quanz, S. P., Henning, Th., Bouwman, J., Linz, H., \& Lahuis, F. 2007, ApJ, 658, 487

Reipurth, B. 1989, Nature, 340, 42

Reipurth, B., \& Aspin, C. 1997, AJ, 114, 2700

Reipurth, B., \& Eiroa, C. 1992, A\&A, 256, L1

Reipurth, B., \& Graham, J. A. 1988, A\&A, 202, 219

Reipurth, B., \& Heathcote, S. 1990, A\&A, 229, 527

Reipurth, B., \& Olberg, M. 1991, A\&A, 246, 535

Reipurth, B., Chini, R., Krügel, E., Kreysa, E., \& Sievers, A. 1993, A\&A, 273, 221

Reipurth, B., Raga, A. C., \& Heathcote, S. 1996, A\&A, 311, 989

Reipurth, B., Bally, J., \& Devine, D. 1997a, AJ, 114, 2708

Reipurth, B., Hartigan, P., Heathcote, S., Morse, J. A., \& Bally, J. 1997b, AJ, 114,757

Reipurth, B., Devine, D., \& Bally, J. 1998, AJ, 116, 1396

Riera, A., López, R., Raga, A. C., Estalella, R., \& Anglada, G. 2003, A\&A, 400, 213

Rodríguez, L. F., \& Hartmann, L. W. 1992, Rev. Mex. Astron. Astrofis., 24, 135 Rodríguez, L. F., \& Reipurth, B. 1994, A\&A, 281, 882

Rodríguez, L. F., Haschick, A. D., Torrelles, J. M., \& Myers, P. C. 1987, A\&A, 186,319

Rodríguez, L. F., Anglada, G., \& Raga, A. 1995, ApJ, 454, L149 
Rodríguez, L. F., Reipurth, B., Raga, A. C., \& Cantó, J. 1998, Rev. Mex. Astron. Astrofis., 34, 69

Sánchez-Monge, A., Palau, A., Estalella, R., Beltrán, M. T., \& Girart, J. M. 2008, A\&A, 485, 497

Sandell, G., \& Weintraub, D. A. 2001, 134, 115

Sandell, G., Hoglund, B., \& Kisliakov, A. G. 1983, A\&A, 118, 306

Sandell, G., Goss, W. M., \& Wright, M. 2005, ApJ, 621, 839

Saraceno, P., Ceccarelli, C., Clegg, P., et al. 1996, A\&A, 315, L293

Sato, F., Mizuno, A., Nagahama, T., et al. 1994, ApJ, 435, 279

Sepúlveda, I. 2001, Ph.D. Thesis, Universitat de Barcelona

Serabyn, E., Güsten, R., \& Mundy, L. 1993, ApJ, 404, 247

Scappini, F., Cecchi-Pestellini, C., Olberg, M., Casolani, A., \& Fanti, C. 1998, ApJ, 504, 866

Schwartz, R. D., Gyulbudaghian, A. L., \& Wilking, B. A. 1991, ApJ, 370, 263

Snell, R. L., \& Bally, J. 1986, ApJ, 303, 683

Snell, R. L., Dickman, R. L., \& Huang, Y.-L. 1990, ApJ, 352, 139

Sridharan, T. K., Beuther, H., Schilke, P., Menten, K. M., \& Wyrowski, F. 2002, ApJ, 566, 931

Stanke, T., McCaugherea, M. J., \& Zinnecker, H. 2000, A\&A, 355, 639

Stapelfeldt, K. R., \& Scoville, N. Z. 1993, ApJ, 408, 239

Staude, H. J., \& Neckel, Th. 1991, A\&A, 244, L13

Staude, H. J., \& Neckel, Th. 1992, ApJ, 400, 556

Strom, K. M., Strom, S. E., Wolff, S. C., Morgan, J., \& Wenz, M. 1986, ApJS, 62,39

Strom, K. M., Newton, G., Strom, S. E., et al. 1989, ApJS, 71, 183

Sugitani, K., Matsuo, H., Nakano, M., Tamura, M., \& Ogura, K. 2000, AJ, 119, 323

Swift, J. J., Welch, W. J., \& Di Francesco, J. 2005, ApJ, 620, 823

Swift, J. J., Welch, W. J., Di FRancesco, J., \& Stojimirović, I. 2006, ApJ, 637, 392

Tafalla, M., Bachiller, R., Wright, M. C. H., \& Welch, W. J. 1997, ApJ, 474, 329

Tamura, M., Ohashi, N., Hirano, N., Itoh, Y., \& Moriarty-Schieven, G. H. 1996, AJ, 112, 2076

Tapia, M., Persi, P., Bohigas, J., \& Ferrari-Toniolo, M. 1997, AJ, 113, 1769

Tatematsu, K., Umemoto, T., Kameya, O., et al. 1993, ApJ, 404, 643

Tofani, G., Felli, M., Taylor, G. B., \& Hunter, T. R. 1995, A\&AS, 112, 299
Torrelles, J. M., Rodríguez, L. F., Cantó, J., et al. 1983, ApJ, 274, 214 Ungerechts, H., Walmsley, C. M., \& Winnewisser, G. 1986, A\&A, 157, 207 Umemoto, T., Hirano, N., Kameya, O., et al. 1991, ApJ, 377, 510

Wang, Y., Evans II, N. J., Zhou, S., \& Clemens, D. P. 1995, ApJ, 454, 217

Weikard, H., Wouterloot, J. G. A., Castets, A., Winnewisser, G., \& Sugitani, K. 1996, A\&A, 309, 581

Weintraub, D. A., \& Kastner, J. 1993, ApJ, 411, 767

Weintraub, D. A., Sandell, G., \& Duncan, W. D. 1991, ApJ, 382, 270

Werner, M. W., Becklin, E. E., Gatley, I., et al. 1979, MNRAS, 188, 463

Wilking, B. A., Mundy, L. G., Blackwell, J. H., \& Howe, J. E. 1989, ApJ, 345, 257

Wilking, B. A., Blackwell, J. H., \& Mundy, L. G. 1990, AJ, 100, 758

Wilking, B. A., Mundy, L. G., McMullin, J., Hezel, T., \& Keene, J. 1993, AJ, 106,250

Williams, S. J., Fuller, G. A., \& Sridharan, T. K. 2005, A\&A, 434, 257

Whittet, D. C. B., Smith, R. G., Adamson, A. J., et al. 1996, ApJ, 458, 363

Whyatt, W., Girart, J. M., Viti, S., Estalella, R., \& Williams, D. A. 2009, A\&A, submitted

Wouterloot, J. G. A., \& Walmsley, C. M. 1986, A\&A, 168, 237

Wouterloot, J. G. A., Brand, J., \& Fiegle, K. 1993, A\&AS, 98, 589

Wu, J., Evans II, N. J., Shirley, Y. L., \& Knez, C. 2010, ApJS, 188, 313

Wu, Y., Henkel, C., Xue, R., Guan, X., \& Miller, M. 2007, 669, L37

Yang, J. 1990, Ph.D. Thesis, Nagoya University

Yang, J., Fukui, Y., Umemoto, T., et al. 1990, ApJ, 362, 538

Yang, J., Umemoto, T., Iwata, T., \& Fukui, Y. 1991, ApJ, 373, 137

Yang, J., Ohashi, N., \& Fukui, Y. 1995, ApJ, 455, 175

Yang, J., Ohashi, N., Yan, J., et al. 1997, ApJ, 475, 683

Yun, J. L., \& Clemens, D. P. 1994a, ApJS, 92, 145

Yun, J. L., \& Clemens, D. P. 1994b, AJ, 108, 612

Yun, J. L., \& Clemens, D. P. 1995, AJ, 109, 742

Yun, J. L., Moreira, M. C., Torrelles, J. M., Afonso, J. M., \& Santos, N. C. 1996, AJ, 111, 841

Zavagno, A., Molinari, S., Tommasi, E., Saraceno, P., \& Griffin, M. 1997, A\&A, 325,685

Ziener, R., \& Eislöffel, J. 1999, A\&A, 347, 565

Zinchencko, I., Henning, Th., \& Schereyer, K. 1997, A\&A, 124, 385

Pages 15 to 27 are available in the electronic edition of the journal at http: //wWw . aanda. org 


\section{Appendix A: Results for individual sources}

\section{A.1. M120.1+3.0-North}

This region is associated with a bipolar molecular outflow (Yang et al. 1990) and contains several low-luminosity objects. Two of these objects, IRAS $00213+6530$ and IRAS $00217+6533$, fall inside the outflow lobes. On the basis of the geometrical position of IRAS $00213+6530$, close to the emission peak of the blue-shifted gas, and its cold IR colors, Yang et al. (1990) favor this source as the driving source of the outflow. We observed the region around both IRAS sources in ammonia.

The $\mathrm{NH}_{3}$ structure (Fig. A.1) consists of two subcondensations, each one peaking very close to the position of an IRAS source. This suggests that both IRAS sources are embedded in the high density gas. Our results show that the velocity is different for each clump (see Table 2 and Fig. A.2). The observed difference in velocity is consistent with a gravitationally bound rotational motion of the two clumps.

The association of IRAS $00213+6530$ with an ammonia emission maximum supports its identification as the outflow exciting source. However, we note that IRAS 00217+6530 falls very close to the position of an ammonia emission peak, it lies close to the emission peak of the outflow redshifted gas and its IRAS colors are characteristic of an embedded source (although the source appears confused in the 60 and $100 \mu$ m IRAS bands). Therefore, based on these results, both IRAS sources are valid candidates for the outflow excitation.

The radio continuum sources detected in the region (Anglada, G., private communication) fall outside the ammonia condensation (see Fig. A.1), therefore they appear to be unrelated to the star-forming region.

\section{A.2. M120.1+3.0-South}

This region is associated with several IRAS sources and with a CO bipolar outflow (Yang et al. 1990). The outflow is asymmetric, with the red lobe more intense than the blue one. Two sources, IRAS 00259+6510 and IRAS 00256+6511, lie inside the outflow lobes. Yang et al. proposed IRAS 00259+6510 as the driving source of the outflow. We observed the region around both sources in ammonia.

The $\mathrm{NH}_{3}$ condensation (Fig. A.3) shows an elongated structure in the NW-SE direction. Both IRAS 00259+6510 and IRAS $00256+6511$ are located close to the ammonia emission maximum, suggesting that they are embedded sources. Both sources have similar IRAS colors, but appear confused at 60 and $100 \mu \mathrm{m}$. Therefore, we cannot favor one of them as the driving source of the outflow.

A $\mathrm{cm}$ radio continuum source is detected at the edge of the ammonia condensation (Anglada, private communication). Unfortunately, the information available for this source is not enough to infer the nature of the emission.

\section{A.3. $L 1287$}

The dark cloud L1287 is associated with an energetic bipolar molecular outflow (Snell et al. 1990; Yang et al. 1991). At the center of the outflow lies the source IRAS 00338+6312, that has been proposed as the outflow exciting source (Yang et al. 1990). The brightest visible object in the region, RNO 1 (Cohen 1980), lies $\sim 40^{\prime \prime} \mathrm{NE}$ of the nominal IRAS position. However, because of the low angular resolution of the IRAS data, several young stellar objects (a FUOri binary system RNO 1B/1C and several

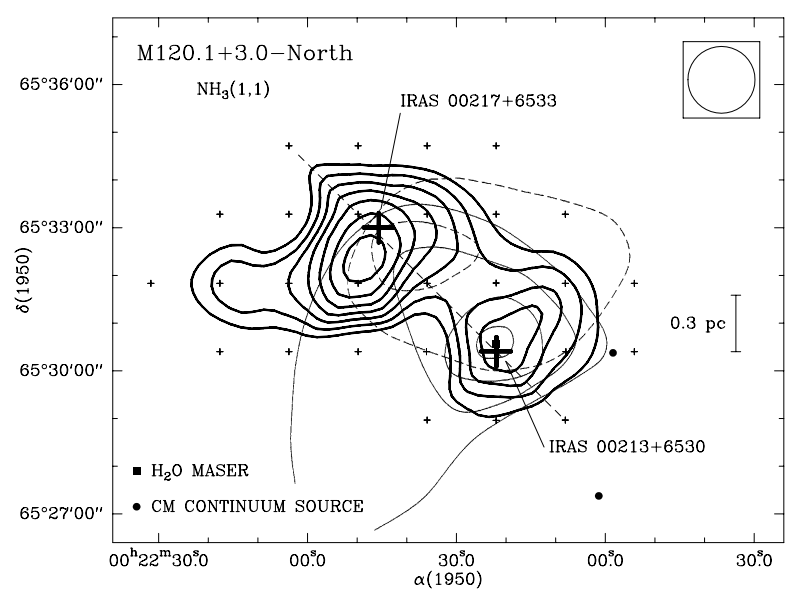

Fig. A.1. Contour map of the main beam brightness temperature of the main line of the ammonia $(J, K)=(1,1)$ inversion transition (thick line) in the M120.1+3.0-North region. The lowest contour level is $0.3 \mathrm{~K}$, and the increment is $0.1 \mathrm{~K}$. The observed positions are indicated with small crosses. The half-power beam width of the telescope is shown as a circle. The positions of several relevant objects in the region are indicated. The CO bipolar outflow (thin line) is from Yang et al. (1990) (solid contours indicate blueshifted gas, and dashed contours indicate redshifted gas). Dashed straight line is the axis of the pos-vel diagram of Fig. A.2.

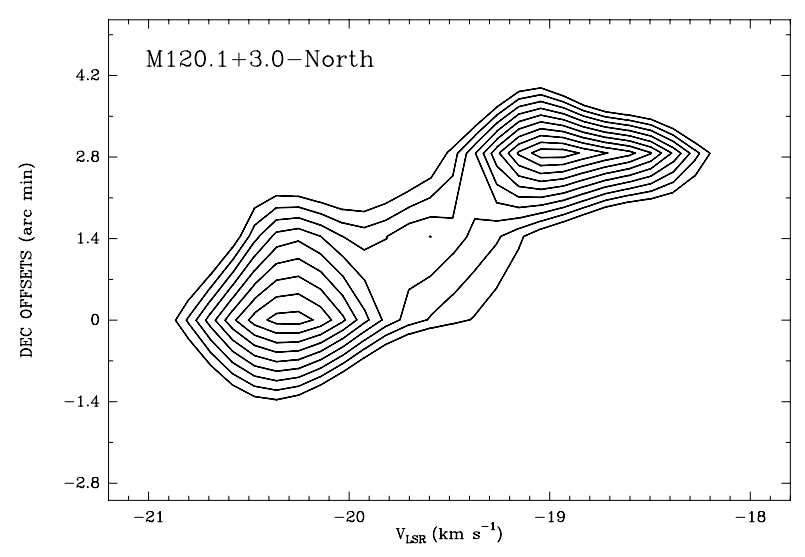

Fig. A.2. Position-velocity diagram of the $\mathrm{NH}_{3}(1,1)$ main line along an axis passing toward the two maxima $\left(\mathrm{PA} \sim 45^{\circ}\right.$ ) of the M120.1+3.0North condensation. The lowest contour level is $0.3 \mathrm{~K}$ and the increment is $0.05 \mathrm{~K}$.

radio continuum sources) fall inside the IRAS error ellipsoid. Kenyon et al. (1993) proposed the FU Ori star RNO 1C as the outflow exciting source. However, additional studies by Anglada et al. (1994) favored a jet-like radio continuum source, VLA 3, located very close to the IRAS nominal position and to the symmetry center of the polarization pattern (Weintraub \& Kastner 1993), as the most likely candidate to be the exciting source. The detection of $\mathrm{H}_{2} \mathrm{O}$ maser emission associated with VLA 3 (Fiebig 1995), and the interpretation of the $\mathrm{H}_{2} \mathrm{O}$ velocity pattern as a infalling disk (Fiebig 1997) further supports VLA 3 as the outflow exciting source.

The region was observed in $\mathrm{HCN}_{1} \mathrm{HCO}^{+}$(Yang et al. 1991), CS (Yang et al. 1995; McMuldroch et al. 1995), and in $\mathrm{NH}_{3}$ (Estalella et al. 1993). Estalella et al. (1993) found a gradient in the NW-SE direction, which was interpreted as caused by the rotation of the core. 


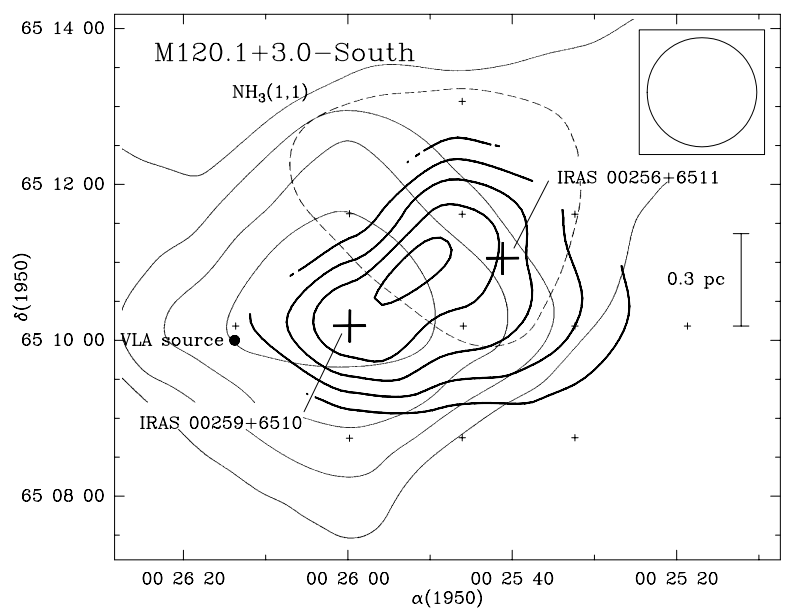

Fig. A.3. Same as Fig. A.1, for the M120.1+3.0-South region. The $\mathrm{NH}_{3}$ lowest contour is $0.2 \mathrm{~K}$ and the increment is $0.1 \mathrm{~K}$. CO bipolar outflow map is also shown (Yang et al. 1990)(solid contours indicate redshifted gas, and dashed contours indicate blueshifted gas).

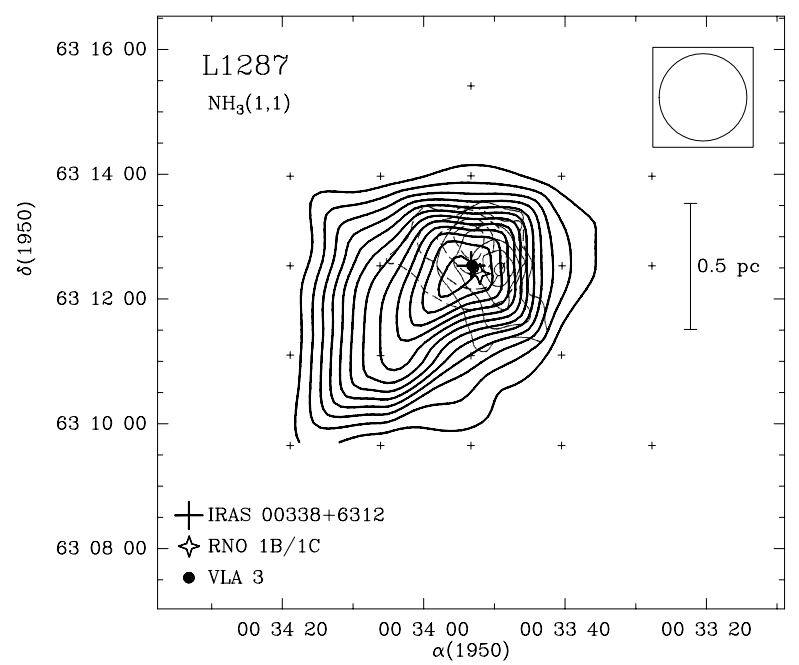

Fig. A.4. Same as Fig. A.1, for the $\mathrm{L} 1287$ region. The $\mathrm{NH}_{3}$ lowest contour is $0.3 \mathrm{~K}$ and the increment is $0.2 \mathrm{~K}$. The map of the $\mathrm{CO}$ bipolar outflow is from Snell et al. (1990).

The condensation we mapped (Fig. A.4) is clearly elongated in the northwest-southeast direction, perpendicular to the $\mathrm{CO}$ outflow axis. We have found a velocity gradient of $\sim 1.23 \mathrm{~km} \mathrm{~s}^{-1} \mathrm{pc}^{-1}$ in the NW-SE direction. Both results agree well with the results of Estalella et al. (1993). The ammonia emission peaks near the position of IRAS $00338+6312$, RNO $1 \mathrm{~B} / 1 \mathrm{C}$ and VLA 3. However, because of the small projected angular separation between all these objects $\left(\sim 5^{\prime \prime}-10^{\prime \prime}\right)$, we cannot distinguish from our data which of these sources is the best candidate for exciting the outflow in terms of its proximity to the ammonia emission peak. This region was studied in $\mathrm{NH}_{3}$ with high angular VLA resolution (Sepúlveda 2001; Sepúlveda et al. in preparation). These observations revealed that the central region toward the sources has a complex structure and exhibits a complex kinematics.

\section{A.4. $L 1293$}

Yang (1990) discovered a bipolar molecular outflow in this region and proposed IRAS $00379+6248$ as its driving source.

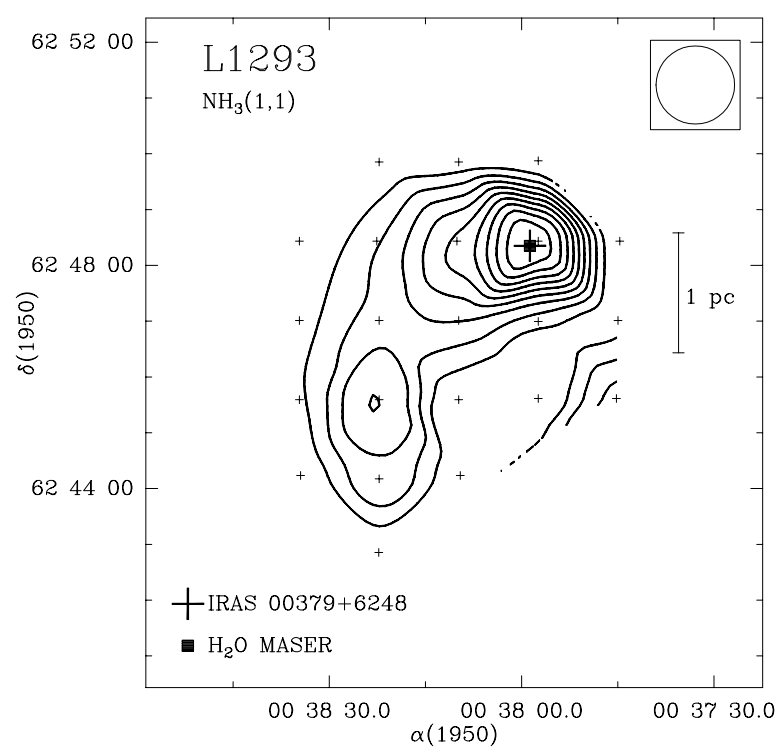

Fig. A.5. Same as Fig. A.1, for the $\mathrm{L} 1293$ region. The $\mathrm{NH}_{3}$ lowest contour level is $0.2 \mathrm{~K}$, and the increment is $0.1 \mathrm{~K}$.

The $\mathrm{NH}_{3}$ structure presents two emission maxima separated by $\sim 4^{\prime}$ (Fig. A.5). The strongest ammonia peak coincides with the position of IRAS $00379+6248$. The $\mathrm{HCN}, \mathrm{HCO}^{+}$ and ${ }^{13} \mathrm{CO}$ emission also peak toward the IRAS position (Yang 1990). This IRAS source is not detected at $12 \mu \mathrm{m}$ and its infrared flux increases steeply toward longer wavelengths. These IR results, along with its association with strong $\mathrm{NH}_{3}$ emission and with an $\mathrm{H}_{2} \mathrm{O}$ maser (Wouterloot et al. 1993), suggest that IRAS $00379+6248$ is a young stellar object, deeply embedded in the high density gas, and the most plausible exciting source of the outflow. The weaker emission peak is not associated with any known object.

\section{A.5. NGC 281 A-West}

This region is associated with a bipolar molecular outflow proposed to be driven by the luminous source IRAS 00494+5617 (Snell et al. 1990; Henning et al. 1994). A near-IR cluster (Carpenter et al. 1993) and several $\mathrm{H}_{2} \mathrm{O}$ maser spots are found in association with the IRAS source (Henning et al. 1992). Henning et al. (1994) modeled the observed spectral energy distribution of the source from $1 \mu \mathrm{m}$ to $1 \mathrm{~mm}$, concluding that it is a very good candidate for a deeply embedded and very young protostellar object.

We detected an ammonia clump (Fig. A.6), which appears unresolved with our beam. The $\mathrm{NH}_{3}$ emission peaks at the position of IRAS $00494+5617$. Our results agree with the $40^{\prime \prime}$ angular resolution $\mathrm{NH}_{3}$ map of Henning et al. (1994), which reveals that the ammonia clump is elongated along the east-west direction with the emission peaking toward the position of the IRAS source. CS emission mapped by Carpenter et al. (1993) with an angular resolution of $\sim 50^{\prime \prime}$, also peaks toward the position of the IRAS source. These results, along with the spectral energy distribution of the source, suggest that IRAS $00494+5617$ is a very young object deeply embedded in the high density gas and that it is the most likely candidate to excite the outflow.

Our ammonia results suggest that there is no significant amount of dense gas in association with the source IRAS $00492+5618$, located $\sim 2^{\prime}$ to the west of IRAS $00494+5617$. 


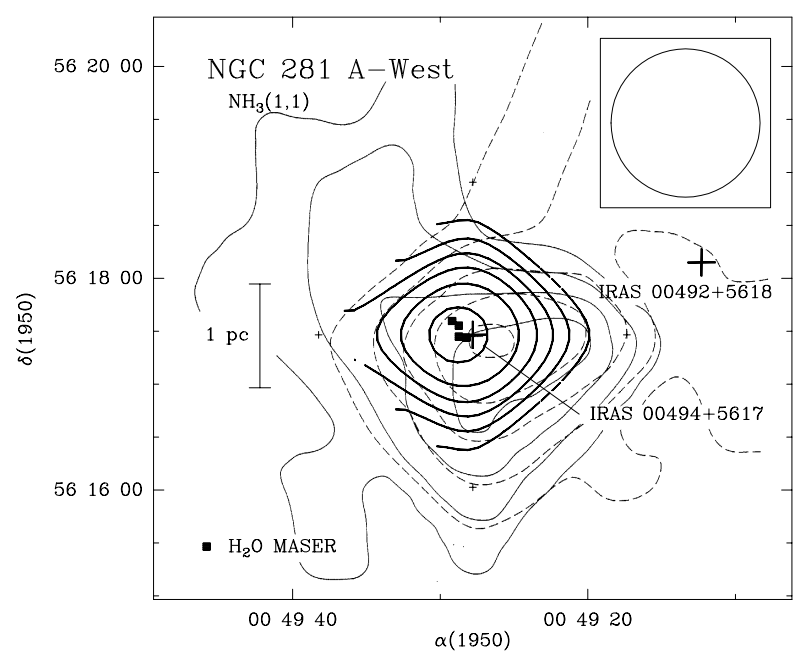

Fig. A.6. Same as Fig. A.1, for the NGC 281 A-West region. The $\mathrm{NH}_{3}$ lowest contour level is $0.15 \mathrm{~K}$, and the increment is $0.075 \mathrm{~K}$. The CO bipolar outflow is from Snell et al. (1990) (solid contours indicate redshifted gas, and dashed contours indicate blueshifted gas).

\section{A.6. $\mathrm{HH} 31$}

The HH 31 jet is a sinusoidal chain of knots having a linear extent of $\sim 0.2$ pc (Herbig 1974; Gómez et al. 1997). Cohen \& Schwartz (1983) found four near-IR sources (IRS1, IRS2, IRS3 and IRS4) in the vicinity of the jet, being IRS 2, that coincides with IRAS $04248+2612$, the proposed exciting source of the jet. This source has been detected at millimeter and submillimeter wavelengths (Moriarty-Schieven et al. 1994) and apparently drives a small molecular outflow (Moriarty-Schieven et al. 1992), although no map has been published. In near-IR images, IRAS 04248+2612 (IRS 2) appears as a bipolar reflection nebula (Padgett et al. 1999). We searched for ammonia emission toward the four near-IR sources.

The ammonia condensation (Fig. A.7) is elongated in the NE-SW direction, and it agrees with the $\mathrm{NH}_{3}$ map shown by Benson \& Myers (1989). The $\mathrm{NH}_{3}$ emission peak is displaced $\sim 3^{\prime}(\sim 0.14 \mathrm{pc})$ to the SW of the HH 31 IRS2 position. To our knowledge, no source has been reported toward the position of the $\mathrm{NH}_{3}$ emission peak. We suggest that high sensitivity observations could reveal a deeply embedded object at this position. The sources HH 31 IRS2 and IRS1 lie at the edge of the condensation. We have not detected significant emission toward IRS3 and IRS4, which lie far away (more than $8^{\prime}$ ) from the condensation.

\section{A.7. $\mathrm{HH} 265$}

HH 265, located in the L1551 cloud, is an isolated Herbig-Haro object whose exciting source still remains unknown. Swift et al. (2005, 2006) mapped the region in $\mathrm{NH}_{3}$ and CS. From their results, these authors suggest that the cloud is likely a prestellar core showing signs of undergoing the first phases of gravitational collapse.

We discovered an $\mathrm{H}_{2} \mathrm{O}$ maser (see Fig. 3) toward the position of the $\mathrm{HH}$ object. The maser shows two velocity components, whose line parameters are given in Table 4. The $\mathrm{H}_{2} \mathrm{O}$ maser emission suggests the presence of a nearby exciting source, which could be also responsible for the excitation of HH 265. However, it is also suggested that HH265 could be the end of

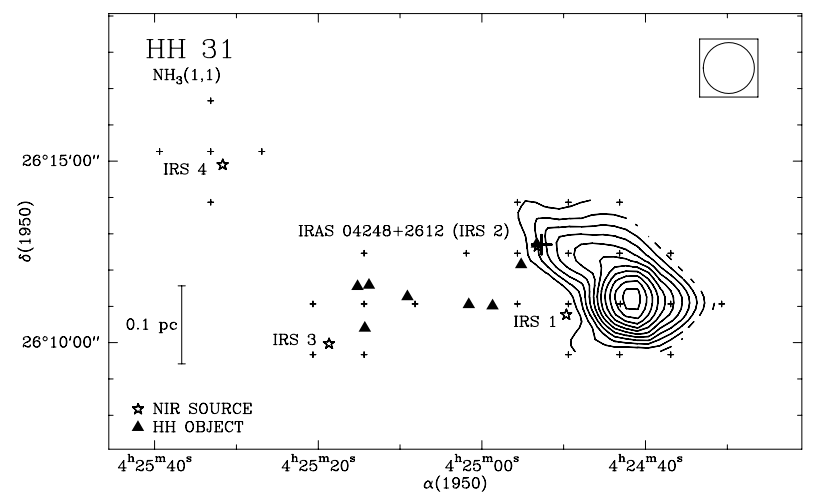

Fig. A.7. Same as Fig. A.1, for the $\mathrm{HH} 31$ region. $\mathrm{The}^{\mathrm{NH}_{3}}$ lowest contour is $0.3 \mathrm{~K}$ and the increment is $0.2 \mathrm{~K}$. The position of the $\mathrm{HH} 31$ knots is from Gómez et al. (1997).

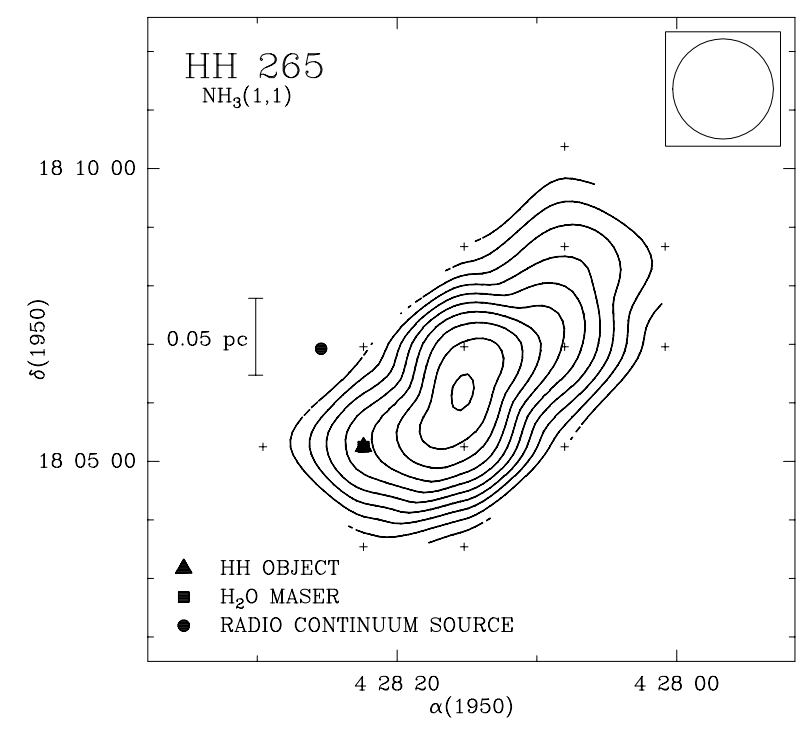

Fig. A.8. Same as Fig. A.1, for the $\mathrm{HH} 265$ region. The $\mathrm{NH}_{3}$ lowest contour is $0.3 \mathrm{~K}$ and the increment is $0.2 \mathrm{~K}$.

a jet emanating from the source $\mathrm{LkH} \alpha 358$ (Moriarty-Schieven et al. 2006; Movsessian et al. 2007).

Our ammonia map (see Fig. A.8) shows that both the $\mathrm{H}_{2} \mathrm{O}$ maser and the $\mathrm{HH}$ object fall inside the ammonia condensation, but they are displaced by $\sim 1.5(\sim 0.08 \mathrm{pc})$ to the SE of the position of the ammonia maximum. We suggest that a sensitive search in the submm, mm, or cm range in the vicinity of the $\mathrm{NH}_{3}$ emission peak could reveal an embedded object, responsible for the excitation of HH 265. The mass derived for this region (see Table 5) exceeds the virial mass by a factor of five, a result that may indicate that a significant fraction of the cloud is still undergoing the process of gravitational collapse toward a central, embedded protostar, in good agreement with the results obtained by Swift et al. $(2005,2006)$.

A $20 \mathrm{~cm}$ source, located $\sim 2^{\prime}$ to the NE of HH 265, was detected by Snell \& Bally (1986). However, this source lies outside the ammonia condensation and was not detected at shorter wavelengths (6 cm and $2 \mathrm{~mm}$; Snell \& Bally 1986), suggestive of a negative spectral index, characteristic of background extragalactic sources. Unfortunately, sensitive observations reaching the position of the ammonia maximum are not available. 


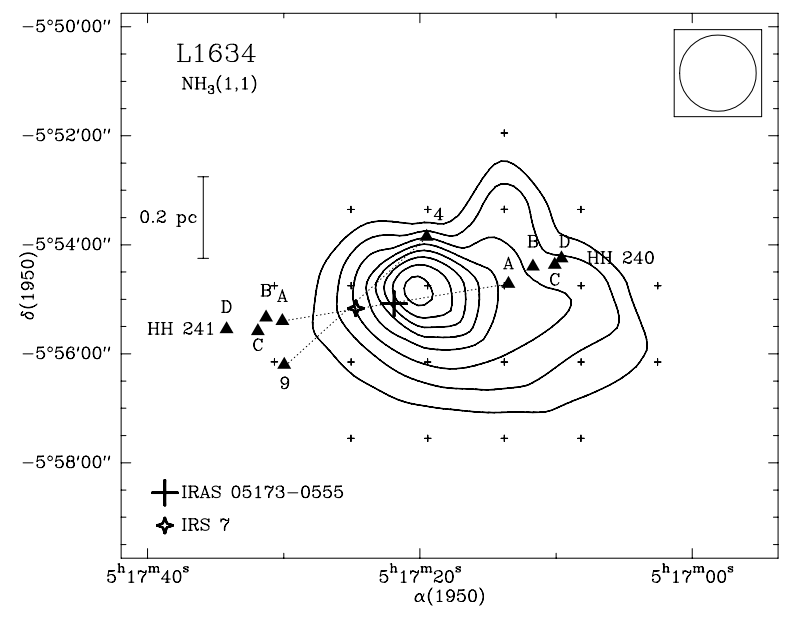

Fig. A.9. Same as Fig. A.1, for the $\mathrm{L} 1634$ region. The $\mathrm{NH}_{3}$ lowest contour is $0.3 \mathrm{~K}$ and the increment is $0.15 \mathrm{~K}$. Dashed lines indicate outflow axes discussed in text. IRAS 05173-0555 is the proposed exciting source for the HH240-241 outflow and the source IRS 7 is the proposed exciting source for the second outflow (knots 4 and 9).

\section{A.8. L1551 NE}

L1551 NE is a young stellar object in the L1551 molecular cloud. It is located very close $(\sim 2.5)$ to the well-studied embedded source L1551 IRS 5. The proximity to the red lobe of the large IRS 5 outflow has made difficult the study of the L1551 NE outflow itself. Moriarty-Schieven et al. (1995) suggest the presence of a weak molecular outflow from this source and Devine et al. (1999) concluded that L1551 NE drives an $\mathrm{HH}$ flow (HH 454) and that probably drives the objects $\mathrm{HH} 28$ and 29 , that were previously attributed to IRS 5.

We detected intense ammonia emission toward the position of L1551 NE (see spectrum in Fig. 1), but the proximity to IRS 5, which is associated with a strong $\mathrm{NH}_{3}$ condensation (Torrelles et al. 1983) makes it difficult to separate both components. Higher angular resolution observations of high density tracers are needed to detect the structure of dense gas around L1551 NE.

\section{A.9. $L 1634$}

L1634 contains two $\mathrm{H}_{2}$ bipolar jets (Hoddap \& Ladd 1995). One of them, $\mathrm{HH} 240-241$, is constituted by several $\mathrm{H}_{2}$ knots symmetrically located from the source IRAS 05173-0555, which has been proposed as the driving source (Hoddap \& Ladd 1995; Davis et al. 1997). The jet extends from east (knots HH 241AD) to west (knots HH 240A-D). Knots HH 240A and HH 241A were previously known as RNO 40 and RNO 40E (Jones et al. 1984). $\mathrm{CO}(J=3 \rightarrow 2$ ) observations (Davis et al. 1997) reveal the presence of a molecular outflow associated with IRAS 05173-0555, a cm and a submm source, proposed as a Class 0 (Beltrán et al. 2002). The second bipolar jet only has two knotty bow shocks (knots 9 and 4; Hoddap \& Ladd 1995; see Fig. A.9). The near-IR source IRS 7 located near the center of the jet, has been proposed as the powering source of this outflow (Hoddap \& Ladd 1995; Davis et al. 1997). The CO outflow (Lee et al. 2000) shows a distribution similar to that of the $\mathrm{H}_{2}$ jets.

Our map (Fig. A.9) shows that both, IRAS 05173-0555 and IRS 7 are associated with dense gas. The $\mathrm{NH}_{3}$ emission peak is located close $(\sim 0.7 \simeq 0.09 \mathrm{pc})$ to the position of the IRAS source. This source has a steeply rising spectral energy distribution through the IRAS bands and it is detected at $\mathrm{cm}$, $\mathrm{mm}$, and submm wavelengths (see references in Table 6). These results together with its association with the ammonia core suggest that IRAS 05173-0555 is a very young object deeply embedded in the high density gas and that it is a very good candidate for exciting the optical jet HH240-241 and the molecular outflow. The near-IR source IRS 7 is displaced by $\sim 1$ '.4 ( $\sim 0.19 \mathrm{pc})$ to the SE of the position of the emission maximum. The association of IRS 7 with high density gas suggests that it is a very young stellar object as suggested by Beltrán et al. (2002).

We noted that the central velocity of the ammonia lines increases to the west of the peak position. We found a velocity shift of $\sim 0.31 \mathrm{~km} \mathrm{~s}^{-1}$ between the ammonia peak position and the position of knot HH 240 A. This knot has a large proper motion away from the IRAS source (Jones et al. 1984). The velocity shift detected could results from the interaction between the jet and the dense gas. Indeed, Whyatt et al. (2009) find that the HH objects are illuminating the molecular gas, enhancing the emission of the $\mathrm{HCO}^{+}$associated with a dense molecular condensation within the ammonia core.

\section{A.10. IRAS $05358+3543$}

IRAS $05358+3543$ was proposed as the exciting source of a bipolar molecular outflow (Snell et al. 1990). Observations at higher angular resolution resolved the outflow into at least three different outflows, two of them forming a quadrupolar system (Beuther et al. 2002a). Tofani et al. (1995) detected four $\mathrm{H}_{2} \mathrm{O}$ maser spots close to the IRAS position. Millimeter and submillimeter emission around the IRAS source resolved at least four cores in the region within separations between $4^{\prime \prime}-6$ " . At least two of these $\mathrm{mm}$ cores will be likely the exciting source of the molecular outflows (Beuther et al. 2007; Leurini et al. 2007).

We found an $\mathrm{NH}_{3}$ condensation elongated in the north-south direction (Fig. A.10). The $\mathrm{NH}_{3}$ emission peaks at the position of the IRAS source. This positional coincidence, as well as its proximity to $\mathrm{H}_{2} \mathrm{O}$ maser emission, along with the fact that its infrared emission increases steeply toward longer wavelengths, suggest that IRAS $05358+3543$ is a very young stellar object, deeply embedded in the high density gas, favoring this object as the driving source of the molecular outflow. Our angular resolution doesn't allow us to infer about the subcores into the region.

\section{A.11. L1641-S3}

L1641-S3 is a bipolar CO outflow located in the southern part of the L1641 cloud (Fukui et al. 1989; Wilking et al. 1990; Morgan et al. 1991). The outflow is centered on the source IRAS 05375-0731 (= FIRSSE-101), which has been proposed as its exciting source. The source has been detected in the nearIR, centimeter, millimeter and submillimeter wavelengths ranges with an spectral energy distribution of a Class I source (see references in Table 6). $\mathrm{H}_{2} \mathrm{O}$ maser emission (Wouterloot \& Walmsley 1986) has been detected toward the IRAS source. An $\mathrm{H}_{2}$ giant flow is found probably associated with the IRAS source (Stanke et al. 2000)

In all the positions where emission is detected, the $\mathrm{NH}_{3}$ spectra show two velocity components at 3.8 and $4.9 \mathrm{~km} \mathrm{~s}^{-1}$, in all the hyperfine lines. Each velocity component peaks at a different position. In Fig. A.11 we show the observed spectra at the position of the emission peak for each velocity component.

The region has also been mapped in $\mathrm{NH}_{3}$ with an angular resolution of $40^{\prime \prime}$ by Harju et al. (1993). These authors present 
I. Sepúlveda et al.: Dense gas and the nature of the outflows

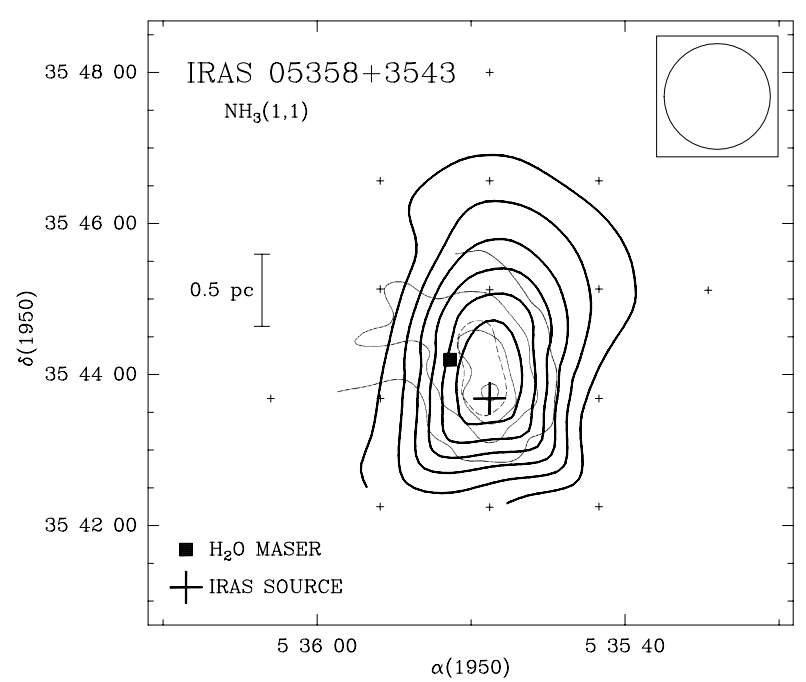

Fig. A.10. Same as Fig. A.1, for the region around IRAS 05358+3543. The $\mathrm{NH}_{3}$ lowest contour is $0.3 \mathrm{~K}$ and the increment is $0.2 \mathrm{~K}$. The $\mathrm{CO}$ bipolar outflow is from Snell et al. (1990).

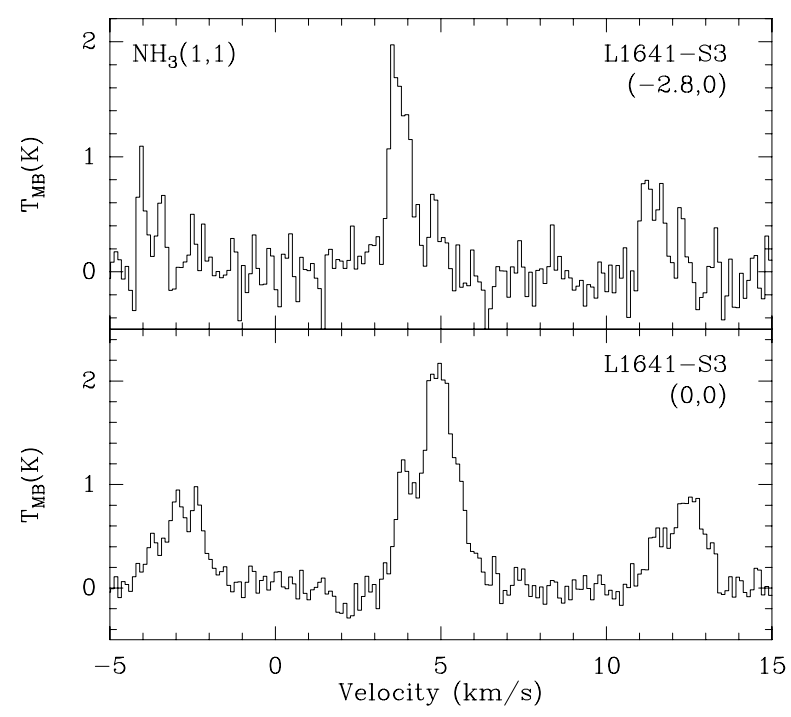

Fig. A.11. Spectra of the $\mathrm{NH}_{3}(1,1)$ emission at $(0,0)$ and $(-2.8,0)$, the positions of the emission maximum for each velocity component of L1641-S3. Offsets are with respect to the position given in Table 1.

a map of the overall emission, which is consistent with our results, taken into account the difference in the beam sizes and the slight difference in the region covered by the maps. However these authors do not discuss the presence of two velocity components. Since the two velocity components are clearly defined in our spectra, in Figs. A.12 and A.13 we present separate maps of the two velocity components, and in our analysis we will discuss separately each velocity component.

The map of the component at $4.9 \mathrm{~km} \mathrm{~s}^{-1}$ (Fig. A.12) reveals a well defined $\mathrm{NH}_{3}$ condensation with the position of source IRAS 05375-0731 well centered in the structure and coinciding with the maximum of emission. The main axis of this condensation is elongated roughly in the NW-SE direction, perpendicular to the outflow axis. We also detected $\mathrm{NH}_{3}$ emission toward the $\mathrm{NE}$ of the region mapped, near the positions of IRAS 05377-0729 and IRAS 05375-0727, suggesting that these sources may be also associated with high density gas. However, none of the two sources is detected at submillimeter wavelengths (Dent et al. 1998) and

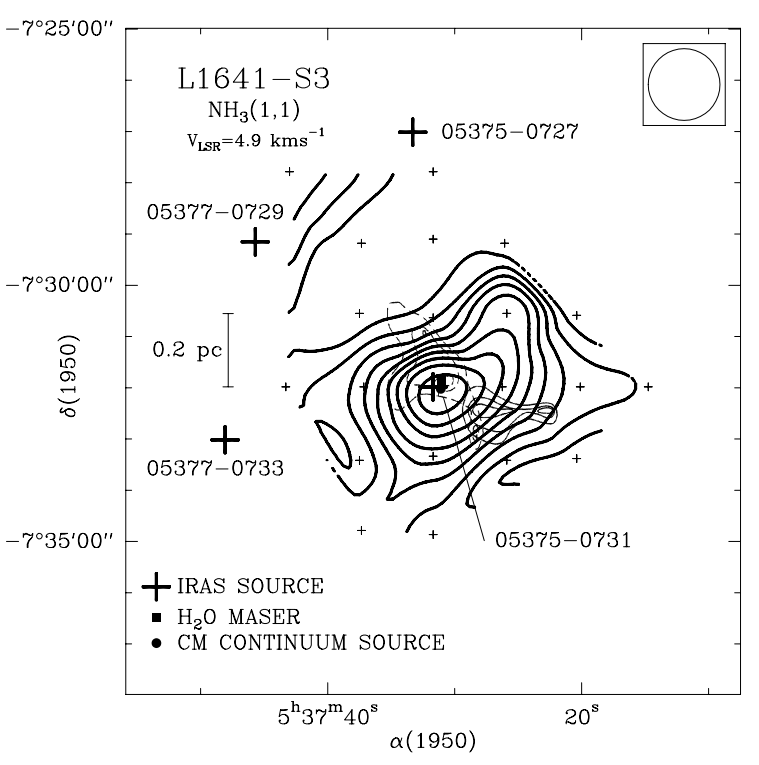

Fig. A.12. Same as Fig. A.1, but for the emission associated with the component at $\sim 4.9 \mathrm{~km} \mathrm{~s}^{-1}$ for the L1641-S3 region. The $\mathrm{NH}_{3}$ lowest contour is $0.3 \mathrm{~K}$ and the increment is $0.2 \mathrm{~K}$. The map of the $\mathrm{CO}$ bipolar outflow is from Morgan et al. (1991).

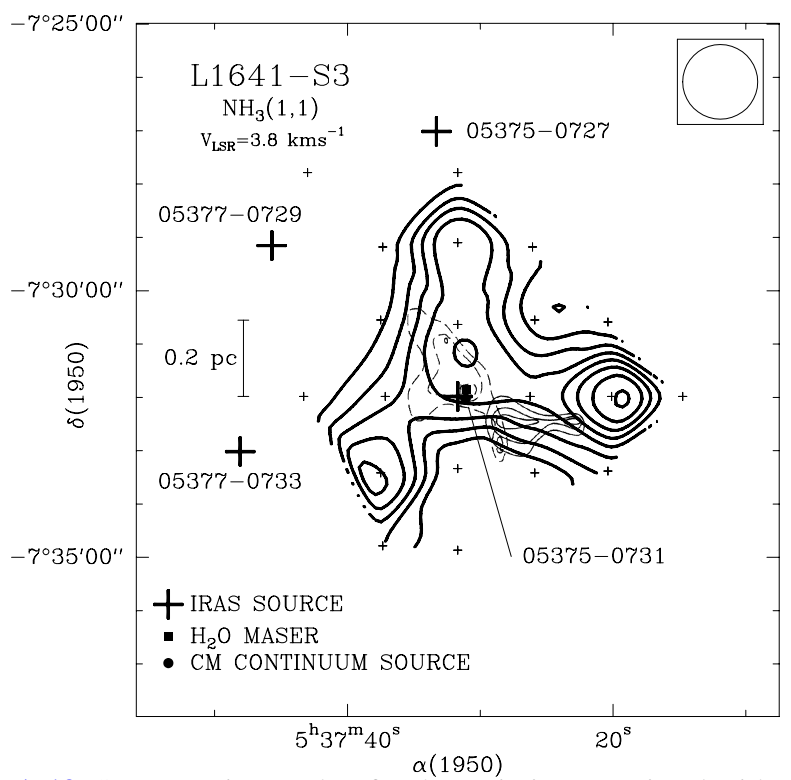

Fig. A.13. Same as Fig.A.1, but for the emission associated with component at $\sim 3.8 \mathrm{~km} \mathrm{~s}^{-1}$ for the L1641-S3 region. The $\mathrm{NH}_{3}$ lowest contour is $0.4 \mathrm{~K}$ and the increment is $0.2 \mathrm{~K}$. The map of the $\mathrm{CO}$ bipolar outflow is from Morgan et al. (1991).

only IRAS $05375-0727$ has a near-IR counterpart (Strom et al. 1989). Unfortunately, our map is not completed around the positions of these sources, so that we cannot establish their association with high density gas.

The map of the component at $3.8 \mathrm{~km} \mathrm{~s}^{-1}$ is shown in Fig. A.13. The spatial distribution presents an irregular morphology, with several local maxima. The source IRAS 05375-0731 appears projected toward this structure, but it is not as clearly associated with any particular feature.

In summary, we observe that for the $4.9 \mathrm{~km} \mathrm{~s}^{-1}$ component, the position of the source IRAS 05375-0731 is better centered on the $\mathrm{NH}_{3}$ structure and closer to the emission maximum than 


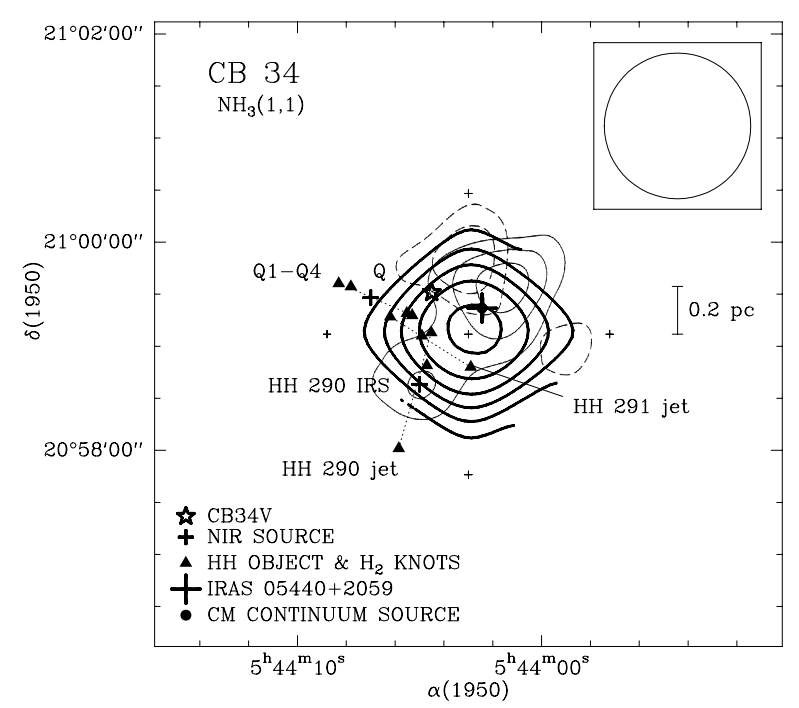

Fig. A.14. Same as Fig. A.1, but for the $\mathrm{CB} 34$ region. The $\mathrm{NH}_{3}$ lowest contour is $0.15 \mathrm{~K}$ and the increment is $0.1 \mathrm{~K}$. The three jets discussed in text are indicated by dotted lines. The map of the $\mathrm{CO}$ bipolar outflow is from Yun \& Clemens (1994a).

for the $3.8 \mathrm{~km} \mathrm{~s}^{-1}$ component. In addition, the main axis of the $4.9 \mathrm{~km} \mathrm{~s}^{-1}$ structure is aligned roughly perpendicular to the outflow axis. Finally, the line widths of the $4.9 \mathrm{~km} \mathrm{~s}^{-1}$ component are broad (see Table 2), suggesting that the dense gas is suffering a perturbation by an embedded object, while the line widths of the $3.8 \mathrm{~km} \mathrm{~s}^{-1}$ component are narrow, suggestive of a starless core. From these results, we conclude that the source IRAS $05375-0731$ is likely associated with the dense gas component at $4.9 \mathrm{~km} \mathrm{~s}^{-1}$. A study of the local heating through a high angular resolution mapping of the $\mathrm{NH}_{3}(1,1)$ and $\mathrm{NH}_{3}(2,2)$ could confirm this association.

\section{A.12. CB 34}

The small Bok globule CB 34 (Clemens \& Barvainis 1988) is associated with the source IRAS $05440+2059$, which is the proposed driving source of a bipolar molecular outflow (Yun \& Clemens 1994a). IRAS 05440+2059 has near-IR, submillimeter, millimeter and centimeter counterparts (see references in Table 6). Near-IR images revealed a small aggregate of YSOs embedded in the cloud (Alves \& Yun 1995). Alves (1995) discovered, from optical and near-IR images, a variable object CB34V, which is identified as an embedded PMS object (Alves et al. 1997).

Moreira \& Yun (1995) discovered in this region four Herbig-Haro objects (HH 290S, HH 290 N1, HH 290 N2 and $\mathrm{HH}$ 291) and several $\mathrm{H}_{2}$ structures (labeled Q1, Q2, Q3, Q4, $h h 291 X$ and $h h 291 Y)$. These authors suggested that the objects HH 290S/N1/N2 constitute an optical jet driven by an embedded near-IR source HH 290 IRS, that the structure Q1-Q4 is a well collimated $\mathrm{H}_{2}$ jet driven by an embedded object (labeled Q), and that $h h 291 X, h h 291 Y$ and HH 291 could be bright knots of an embedded jet, whose driving source remains undetected.

The ammonia structure, unresolved with our beam, peaks close to the position of IRAS $05440+2059$ (see Fig. A.14), in good agreement with the results obtained from other highdensity tracers (CS, Launhardt et al. 1998; HCN, Afonso et al. 1998; $\mathrm{NH}_{3}$, Codella \& Scappini 1998). The sources HH 290 IRS and CB34V appear in projection toward the ammonia core,

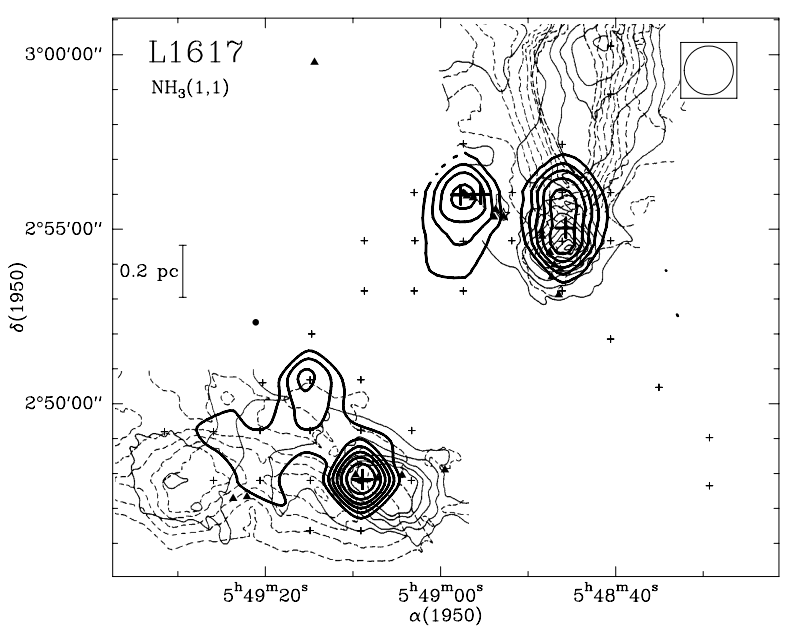

Fig. A.15. Ammonia cores in L1617 (thick contours) overlapped on the CO outflow maps of Reipurth \& Olberg (1991) (thin contours). Symbols used in this map are same as Fig. A.1. Close-ups of the clumps of the NW region (associated with HH 270/110) and SE region (associated with HH 111) are shown in Fig. A.16 and Fig. A.17 respectively. Ammonia contour levels are the same as in these figures. Additional positions were observed near HH $113\left(\sim 12^{\prime}\right.$ east from HH111).

suggesting that they are young stellar objects embedded in the dense molecular gas. These results support the identification of IRAS $05440+2059$ as the exciting source of the molecular outflow, and HH290 IRS as the exciting source of an optical jet. The source Q lies at the edge of the condensation. The HH 291 jet, two of whose knots are only detected in the near-IR, also appears projected toward the high density gas, so it can be tracing an embedded jet as proposed by Moreira \& Yun (1995). We suggest that the exciting source of this jet could be located in the line connecting the knots and close to the position of the $\mathrm{NH}_{3}$ emission maximum. High-resolution observations could reveal the position of this embedded object.

\section{A.13. $L 1617$}

A map of the overall L1617 region where $\mathrm{NH}_{3}$ emission is detected is shown in Fig. A.15. The map encloses the ammonia condensations associated with HH 270/110 and with HH 111, as well as their molecular outflows.

\section{A.13.1. $\mathrm{HH} 270 / 110$}

HH 110 is a well collimated jet located in the L1617 molecular cloud (Reipurth \& Olberg 1991). Reipurth et al. (1996) discovered a second jet, HH 270, $\sim 3^{\prime}$ to the NE of HH 110 and proposed the near-IR source HH 270 IRS as its exciting source. The position of HH 270 IRS lies very close to the error ellipsoid of IRAS $05489+0256$ and both sources could be associated. VLA observations of HH 270 IRS at $\mathrm{cm}$ wavelengths (Rodríguez et al. 1998) revealed that this source (named VLA1) is elongated along the axis of the $\mathrm{HH} 270$ jet, suggesting that it traces the base of the flow. Reipurth et al. (1996) suggest that the HH 270 jet suffers a grazing collision with a nearby molecular cloud core, thus producing a deflected flow, which is manifested as the HH 110 jet. The kinematical studies (Riera et al. 2003; López et al. 2005) provide some additional evidence of the interaction between the outflow and the surrounding material. 


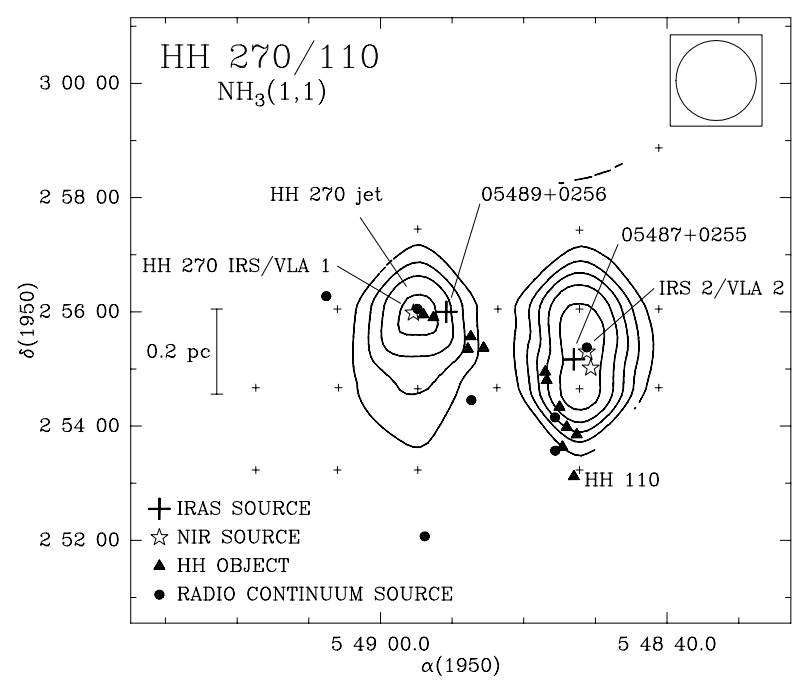

Fig. A.16. Same as Fig. A.1, for the $\mathrm{HH} 270 / 110$ region. The $\mathrm{NH}_{3}$ lowest contour level is $0.2 \mathrm{~K}$ and the increment is $0.15 \mathrm{~K}$. The sources discussed in text are indicated.

About $3^{\prime}$ to the SW of HH 270 IRS, lies IRAS $05487+0255$, a source with a spectral energy distribution steeply rising toward longer wavelengths. IRAS $05487+0255$ is associated with a near-IR and a VLA source (Davis et al. 1994; Garnavich et al. 1997; Rodríguez et al. 1998), driving a bipolar molecular outflow (Reipurth \& Olberg 1991; see Fig. A.15) and a $\mathrm{H}_{2}$ jet (Davis et al. 1994; Garnavich et al. 1997) running almost north-south. A second near-IR source, powering another $\mathrm{H}_{2}$ jet extending in the north-south direction, is found a few arcseconds to the south (Davis et al. 1994; Garnavich et al. 1997).

Our ammonia map (Fig. A.16) shows two high density clumps, separated by $\sim 0.4 \mathrm{pc}$, apparently corresponding with two local maxima observed in the ${ }^{13} \mathrm{CO}$ extended structure mapped by Reipurth et al. (1996). The emission of the eastern $\mathrm{NH}_{3}$ clump peaks at the position of HH 270 IRS/VLA 1, suggesting that this object is embedded in the high density gas, and giving support to its identification as the powering source of the HH 270 jet.

The HH 110 flow is observed toward the SE edge of the western ammonia clump (see Fig. A.16). The coincidence of the $\mathrm{NH}_{3}$ clump at the point where the HH 270/110 flow changes abruptly its direction, gives strong support to the scenario proposed by Reipurth et al. (1996), where the HH 110 jet arises as a result of the deflection of the HH 270 jet after a collision with a highdensity clump. Our $\mathrm{NH}_{3}$ observations provide evidence for the presence of such a high-density clump.

The western $\mathrm{NH}_{3}$ clump peaks near the positions the proposed exciting sources of the molecular outflow and the $\mathrm{H}_{2}$ jets (see Fig. A.16). This result suggest that these sources are embedded objects, giving support to their identification as the driving sources of the molecular outflow and the $\mathrm{H}_{2}$ jets.

The remaining five centimeter continuum sources detected in the region by Rodríguez et al. (1998, see Fig. A.16) have negative spectral index, characteristic of non-thermal emission. One of them (VLA 4) could be associated with the knot HH $110 \mathrm{H}$ and the others are probably background objects unrelated with the star-forming region.

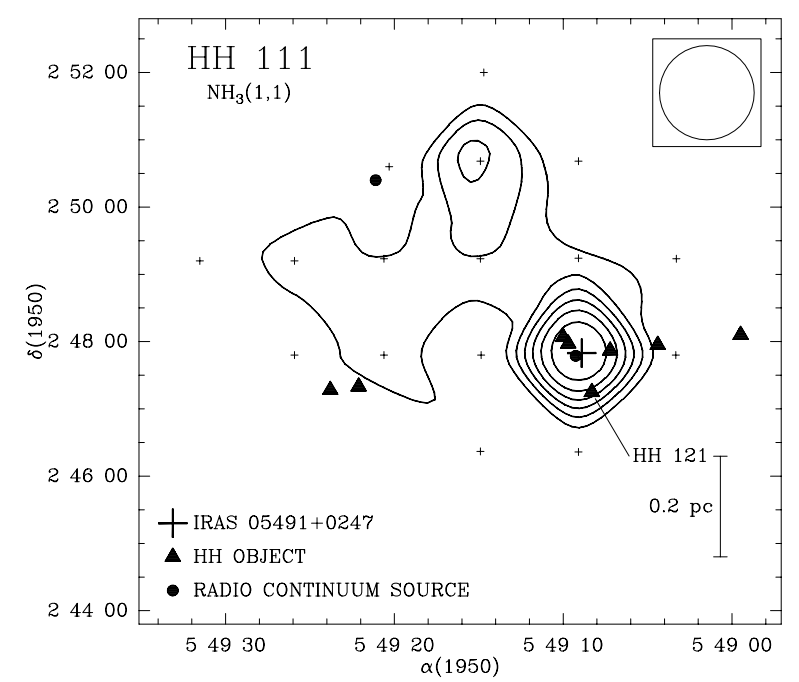

Fig. A.17. Same as Fig. A.1, for the $\mathrm{HH} 111$ region. The $\mathrm{NH}_{3}$ lowest contour level is $0.15 \mathrm{~K}$ and the increment is $0.07 \mathrm{~K}$.

\section{A.13.2. $\mathrm{HH} 111$}

HH 111, in the L1617 cloud, is a well collimated jet associated with a highly collimated molecular outflow apparently driven by IRAS 05491+0247 (Reipurth \& Oldberg 1991; see Fig. A.15), which also has a centimeter counterpart, VLA 1 (Rodríguez \& Reipurth 1994, Anglada et al. 1998b). Reipurth et al. (1997b) proposed that the HH 111 jet (together with HH 113 and HH 311) constitutes a giant flow with a total extent of $7.7 \mathrm{pc}$. Gredel \& Reipurth (1993) detected an $\mathrm{H}_{2}$ bipolar jet, $\mathrm{HH}$ 121, which is almost perpendicular to $\mathrm{HH} 111$ and appears to emanate from the IRAS/VLA 1 source. Cernicharo \& Reipurth (1996) resolved the $\mathrm{CO}$ outflow into a quadrupolar structure along the axes of the HH111 and HH 121 jets. At smaller scales, the source VLA 1 also shows evidence of a similar quadrupolar structure (Reipurth et al. 1999). These authors detected an additional centimeter source, VLA 2 ( $3^{\prime \prime}$ NW of VLA 1$)$, which exhibits some evidence of driving its own outflow.

The $\mathrm{NH}_{3}$ map (Fig. A.17) shows a condensation with the emission peaking near the positions of the proposed triple system. The spectral energy distribution of the IRAS source is steeply rising toward longer wavelengths. Altogether this suggests that the sources are deeply embedded in the high density gas.

The remaining radio continuum sources detected in the region (Anglada et al. 1998b) are not associated with dense gas and have negative spectral index, indicating that probably almost all are non-thermal background sources unrelated to the star-forming region.

\section{A.13.3. $\mathrm{HH} 113$}

We observed a five-point grid around HH 113 (not shown in Fig. A.15), which is located $\sim 12^{\prime}$ to the east of the HH 111 complex. We did not detect significant emission in any of these positions. Reipurth et al. (1997a) suggest that HH 113 is the eastern boundary of the HH 111 complex. The lack of dense gas around this object, and that there are no sources in its vicinity suggests a non local origin for this object, giving support to its identification as part of the HH 111 complex. 


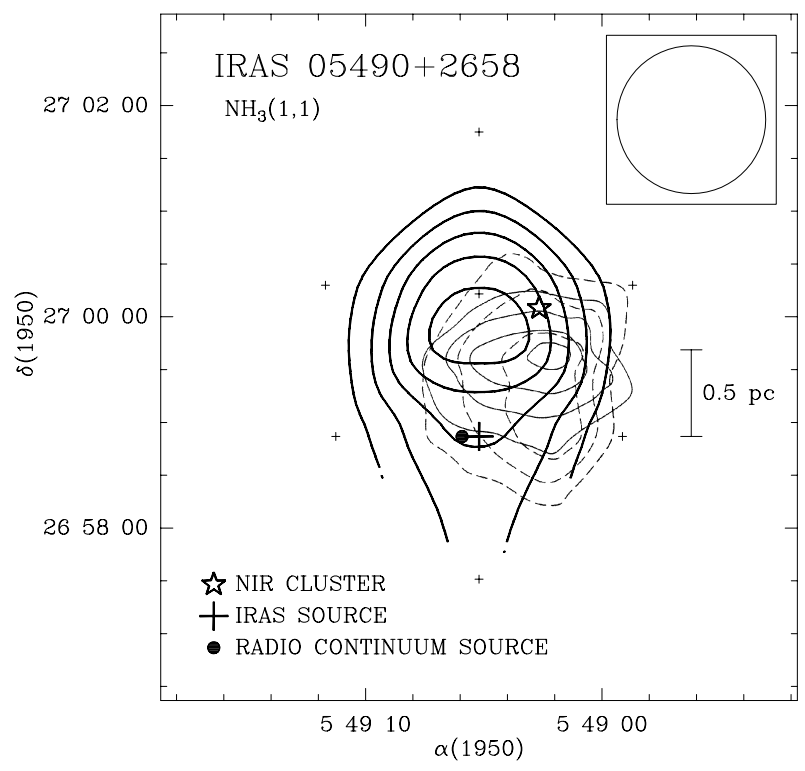

Fig. A.18. Same as Fig. A.1, for the region around IRAS 05490+2658. The $\mathrm{NH}_{3}$ lowest contour is $0.2 \mathrm{~K}$ and the increment is $0.1 \mathrm{~K}$. The center of the near-IR cluster (Carpenter et al. 1993), which extends over a region of $1 \mathrm{pc}$ in size, is indicated. The CO bipolar outflow is from Snell et al. (1990).

\section{A.14. IRAS $05490+2658$}

IRAS $05490+2658$ lies $\sim 5^{\prime}$ east of the H II region S242. This IRAS source has been proposed as the exciting source of a poorly collimated molecular outflow (Snell et al. 1990), although it is displaced $\sim 1^{\prime}$ to the SE of the geometrical center of the outflow. A $6 \mathrm{~cm}$ radio continuum source has been detected close to the position of the IRAS source (Carpenter et al. 1990). A nearIR cluster, extending over a region of $\sim 1 \mathrm{pc}$ in size, has been detected in the region by Carpenter et al. (1993).

The condensation we mapped in $\mathrm{NH}_{3}$ (Fig. A.18) has the emission peak displaced $\sim 1^{\prime}(\sim 0.7 \mathrm{pc})$ to the north of the IRAS source position, but it is very close to the center of the outflow and to the center of the near-IR cluster. This result suggests that some cluster members could be embedded stellar objects, in agreement with the Carpenter et al. (1993) suggestion, and that the outflow exciting source could be located close to the $\mathrm{NH}_{3}$ maximum and to the north of the IRAS position. Sensitive $\mathrm{cm}$ continuum observations toward this position could reveal this object.

\section{A.15. CB 54}

CB 54 is a Bok globule associated with the source IRAS 070201618 , which has been proposed as the exciting source of a highly collimated bipolar molecular outflow (Yun \& Clemens 1994a). The IRAS source is double in the near-IR (two components CB54YC1-I and CB54YC1-II separated by 12": Yun \& Clemens 1994b) and it is detected also at cm (Yun et al. 1996; Moreira et al. 1997) and mm wavelengths (Launhardt \& Henning 1997). Only CB54YC1-II is detected in the mid-infrared images, but three new mid-infrared sources with no near-infrared counterpart were detected spatially coincident with both the IRAS source and the center of the dense core (Ciardi \& Gómez Martín 2007).

We found a compact $\mathrm{NH}_{3}$ condensation (Fig. A.19) with the emission peaking at the position of the IRAS source, which has a $\mathrm{cm}$ radio continuum counterpart. The spectral energy

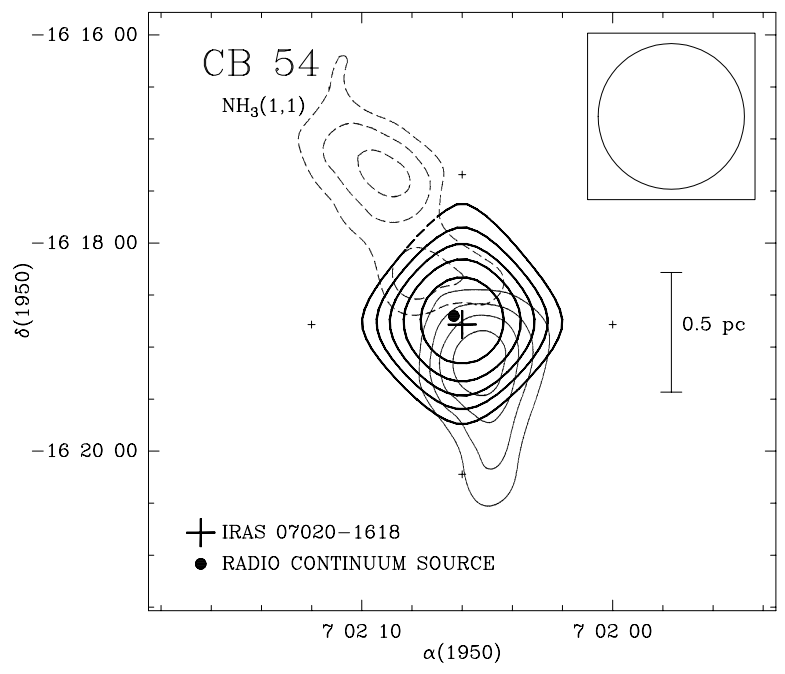

Fig. A.19. Same as Fig. A.1, for the $\mathrm{CB} 54$ region. The $\mathrm{NH}_{3}$ lowest contour is $0.25 \mathrm{~K}$ and the increment is $0.1 \mathrm{~K}$. The $\mathrm{CO}$ bipolar outflow is from Yun \& Clemens (1994a).

distribution of the IRAS souce is steadily rising at longer wavelengths. Altogether this suggests that the IRAS source is a very young object deeply embedded in the high density gas and favors it as the exciting source.

\section{A.16. L379}

The dark cloud L379 contains the bright source IRAS 182651517 , which was proposed as the exciting source of a bipolar molecular outflow (Hilton et al. 1986; Wilking et al. 1990). The red- and blue-wing emission overlap for most of the outflow extension, but the emission maxima are not coincident. This structure has been interpreted as two outflows centered north and south of the IRAS source (Kelly \& McDonald 1996). Observations at $\mathrm{mm}$ and submm wavelengths have revealed two distinct clumps of dust continuum emission located several arcsecs northwest and southwest, respectively from the IRAS nominal position. This interpretation is supported by the two velocity components found in $\mathrm{C}^{18} \mathrm{O}$ spectra (McCutcheon et al. 1995; Kelly \& Mcdonald 1996). Kelly \& McDonald (1996) suggest that the dust clumps probably contain the driving sources of the molecular outflows.

We found an $\mathrm{NH}_{3}$ condensation (Fig. A.20) with the emission peaking at the position of IRAS 18265-1517. The spectral energy distribution of this IRAS source rises steeply at longer wavelengths. Altogether this suggests that the IRAS source is a deeply embedded object. Although our ammonia lines are broad, a hint of two velocity components can be appreciated in the satellite lines (see Fig. 1), in agreement with the $\mathrm{C}^{18} \mathrm{O}$ results (Kelly \& McDonald 1996). Both submm sources appear to be associated with $\mathrm{NH}_{3}$ emission, but our angular resolution does not allow us to favor one of them in terms of the proximity to the $\mathrm{NH}_{3}$ maximum.

The physical parameters we obtained for this region (Table 5) indicate that $\mathrm{L} 379$ is a massive region $(M \sim$ 2000-3700 $M_{\odot}$ ). The estimated luminosity of the IRAS source is $L_{\text {bol }} \sim 1.6 \times 10^{4} L_{\odot}$ (Kelly \& McDonald 1996). The high mass obtained from $\mathrm{NH}_{3}$, together with the high luminosity of the source could indicate that this source is a massive protostellar object, and thus that L379 is a high-mass star-forming region. 


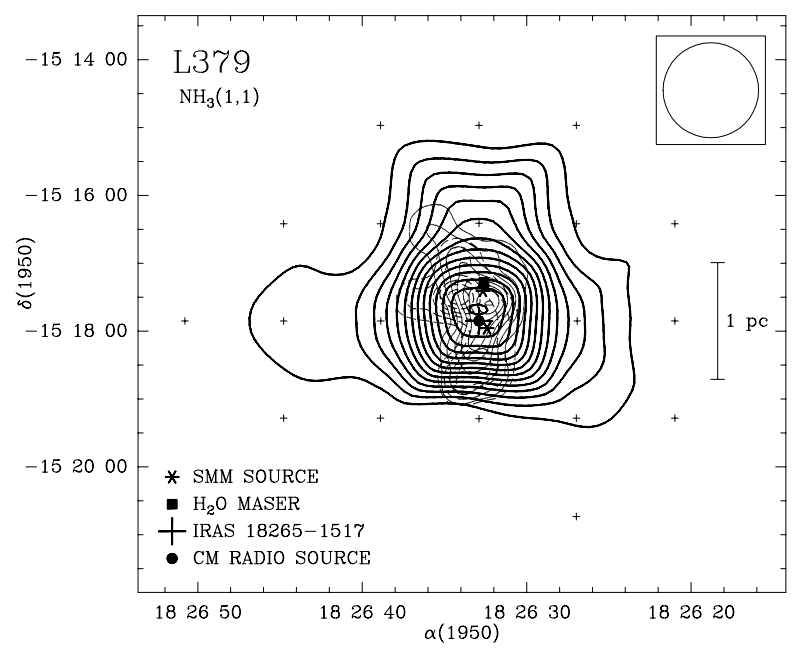

Fig. A.20. Same as Fig. A.1, for the $\mathrm{L} 379$ region. The $\mathrm{NH}_{3}$ lowest contour level is $0.3 \mathrm{~K}$ and the increment is $0.2 \mathrm{~K}$. The position of the two dust clumps is indicated by an asterisk. The map of the CO bipolar outflow is from Kelly \& Macdonald (1996).

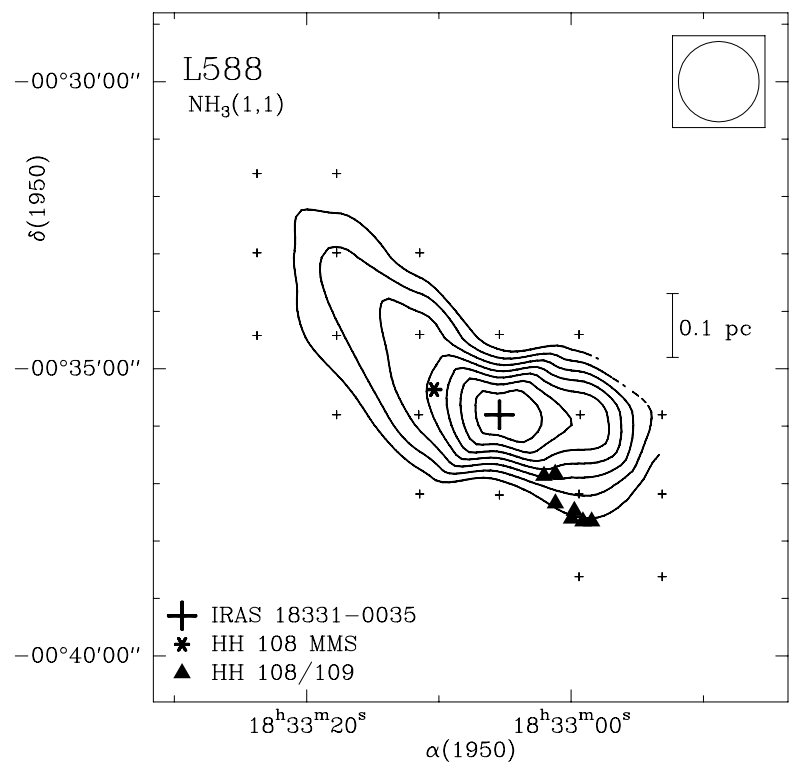

Fig. A.21. Same as Fig. A.1, for the $\mathrm{L} 588$ region. The $\mathrm{NH}_{3}$ lowest contour is $0.3 \mathrm{~K}$ and the increment is $0.15 \mathrm{~K}$. The position of the HH 108 and HH 109 knots are from Ziener \& Eislöffel (1999).

\section{A.17. $L 588$}

Reipurth \& Eiroa (1992) discovered two isolated Herbig-Haro objects, HH 108 and $\mathrm{HH}$ 109, in this region and proposed IRAS 18331-0035 as their driving source. Ziener \& Eislöffel (1999) found that both $\mathrm{HH}$ objects consist of several bright knots, some of them with $\mathrm{H}_{2}$ counterpart. Chini et al. (1997) found two $1.3 \mathrm{~mm}$ sources, the stronger is coincident with the IRAS source and the fainter, HH 108 MMS, has no counterpart. At present it is unclear which one of these two sources is the driving source of the $\mathrm{HH}$ objects. Parker et al. (1991) detected broad line wings in CO spectra taken toward the IRAS position.

The $\mathrm{NH}_{3}$ map (Fig. A.21) shows a condensation elongated in the NE-SW direction, similarly to the molecular cloud mapped in CO by Parker et al. (1991). The ammonia emission maximum is located at the position of IRAS 18331-0035. This result, along with the spectral energy distribution, suggests that

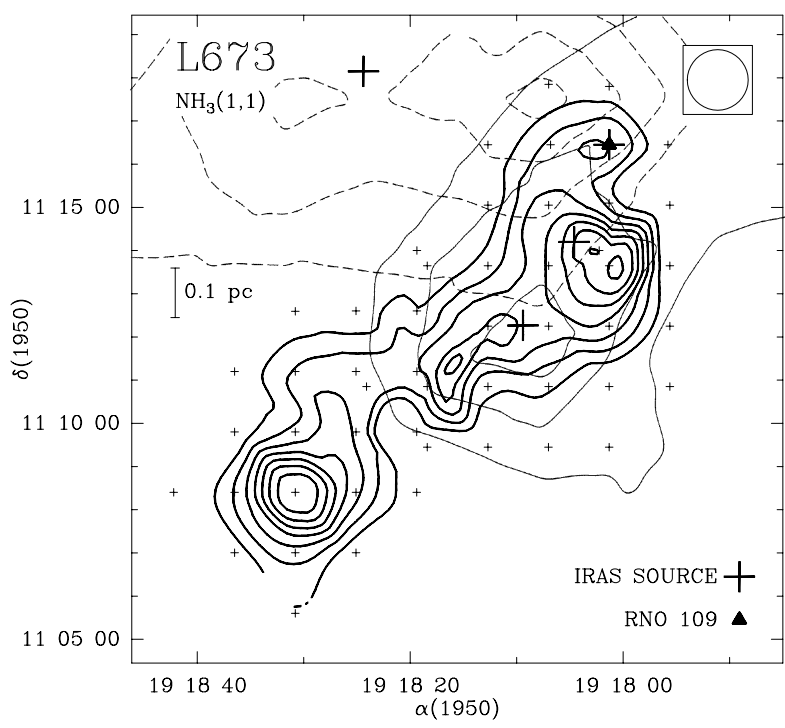

Fig. A.22. Same as Fig. A.1, but for the L673 region. The $\mathrm{NH}_{3}$ lowest contour level is $0.45 \mathrm{~K}$ and the increment is $0.3 \mathrm{~K}$. The $\mathrm{NH}_{3}$ map obtained in previous observations is also included. The IRAS sources associated with the ammonia structure are (from north to south) IRAS $19180+1116(=$ RNO 109), IRAS $19180+1114$ and IRAS 19184+1118. The map of the CO molecular outflow is from Armstrong \& Winnewisser (1989).

the IRAS source is a very young object deeply embedded in the high-density gas. Although the source HH $108 \mathrm{MMS}$ is located inside the $\mathrm{NH}_{3}$ condensation, it is displaced $\sim 1.2(\sim 0.1 \mathrm{pc})$ to the NE of the emission peak. Owing to its association with the $\mathrm{NH}_{3}$ emission peak, it appears that the source IRAS 18331-0035 is the deepest embedded object and constitutes a very good candidate for the energy source of the HH complex.

From our data we found that the mass of this region exceeds the virial mass by more than a factor of five (see Table 5). This result could indicate that the cloud is in process of gravitational collapse.

\section{A.18. $L 673$}

Armstrong \& Winnewisser (1989) detected an extended bipolar molecular outflow in this region and proposed the source IRAS 19180+1116, which coincides with the object RNO 109 (Cohen 1980), as its driving source. In a previous work (see Paper I) we have observed in $\mathrm{NH}_{3}$ a region of $\sim 10^{\prime} \times 7^{\prime}$ around the object RNO 109. Those observations revealed an ammonia structure of $\sim 5^{\prime} \times 2^{\prime}$ elongated from northwest to southeast. It consists of three subcondensations peaking at the positions of sources IRAS 19180+1116 (RNO 109), IRAS 19180+1114 and IRAS $19181+1114$. The source IRAS $19180+1114$ is located close to the strongest emission maximum.

The region was mapped in CS by Morata et al. (1997). The CS emitting region is elongated in the NW-SE direction and is more extended $\left(16.6 \times 7 \mathbf{7}^{\prime} 6\right)$ than the region mapped in ammonia in Paper I. This extended CS emission encompasses the $\mathrm{NH}_{3}$ condensation, and has the emission peak displaced $\sim 8^{\prime}$ to the south-east of IRAS 19180+1114.

To complete the study in $\mathrm{NH}_{3}$ of this region, we carried out new observations, covering the region around the CS emission maximum. In Fig. A.22 we show the complete $\mathrm{NH}_{3}$ map of the region (including the data from Paper I). The new observations reveal that the $\mathrm{NH}_{3}$ emission further extends to the $\mathrm{SE}$, where 


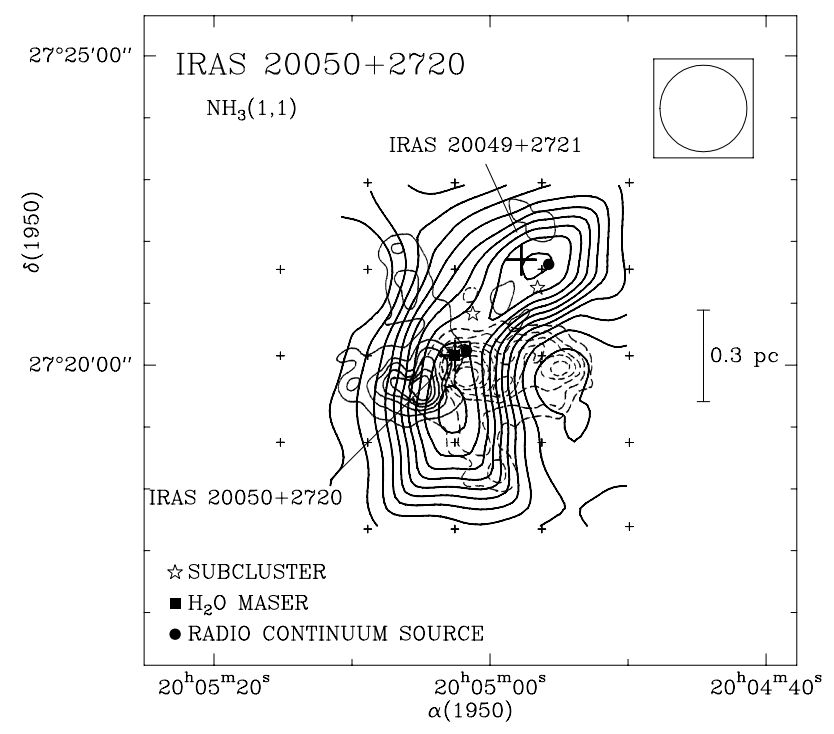

Fig. A.23. Same as Fig. A.1, but for the region around IRAS $20050+2720$. The $\mathrm{NH}_{3}$ lowest contour level is $0.4 \mathrm{~K}$ and the increment is $0.15 \mathrm{~K}$. The centers of the three near-IR subclusters found by Chen et al. (1997) are indicated. The map of the CO multipolar outflow is from Bachiller et al. (1995).

we found the strongest $\mathrm{NH}_{3}$ maximum of the whole region. Up to now, no source has been found toward this position. Morata, Girart \& Estalella $(2003,2005)$ have found that this core splits in multiple condensations, with no signs of star formation. Most of the condensations are transient, in the sense that they are not gravitationally bound.

\section{A.19. IRAS $20050+2720$}

Bachiller et al. (1995) mapped a molecular outflow consisting of three pairs of lobes emanating from the vicinity of IRAS 20050+2720, suggesting that two or three independent outflows are driven by young sources embedded in the core. Chen et al. (1997) found a cluster of near-IR sources with three subclusters, two of them are associated with IRAS 20050+2720 and IRAS 20049+2721, respectively. IRAS 20050+2720 was resolved at centimeter wavelengths in two components at subarsec scale (Anglada et al. 1998a) and in four sources at millimeter wavelengths. Two of them, which are separated $\sim 20^{\prime \prime}$, are suggested to be protostellar collapse candidates (Choi et al. 1999; Beltrán et al. 2008). An analysis of the velocity fields of $\mathrm{H}_{2} \mathrm{O}$ masers (Furuya et al. 2005) indicates that one of these millimeter sources (MM1) is driving a powerful jet. IRAS 20049+2721 was barely detected in the IRAS 12 and $25 \mu \mathrm{m}$ bands ( $2.3 \mathrm{Jy}$ in both bands), but is very bright at longer wavelengths (flux densities are 171.2 and 397.7 Jy in the 60 and $100 \mu \mathrm{m}$ IRAS bands, respectively). A cm continuum source (Anglada et al. 1998a) is detected in association with this source. CS observations show the emission maximum at the position of IRAS 20050+2720, while only weak CS emission was detected toward IRAS 20049+2721 (Bachiller et al. 1995)

The $\mathrm{NH}_{3}$ condensation (Fig. A.23) shows two strong emission peaks, very close to the positions of the IRAS sources of the region, suggesting that both sources are associated with high density gas. The velocities of the two $\mathrm{NH}_{3}$ maxima differ by $\sim 2 \mathrm{~km} \mathrm{~s}^{-1}$ (see Table 2). In Fig. A.24 we show a positionvelocity diagram along the northwest-southeast direction. The

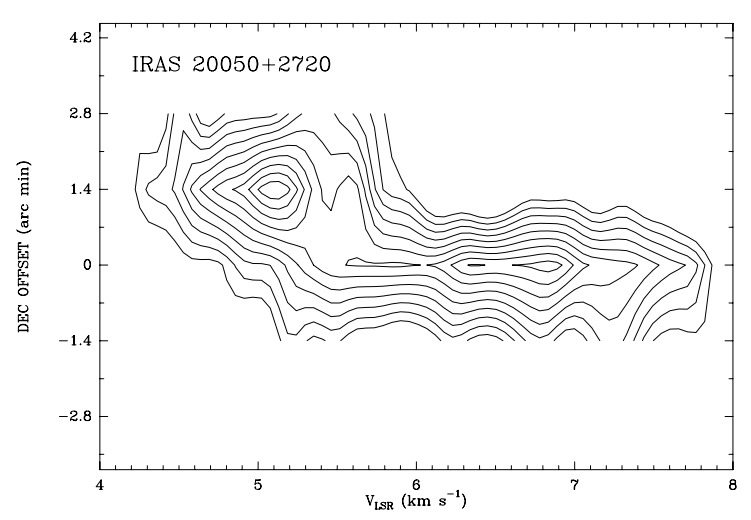

Fig. A.24. Position-velocity diagram of the $\mathrm{NH}_{3}$ main line along the NW-SE direction $\left(\right.$ P.A. $=-45^{\circ}$ ) centered on IRAS $20050+2720$. The 1.4 offset corresponds to the position of IRAS 20049+2721. The lowest contour level is $0.4 \mathrm{~K}$ and the increment is $0.15 \mathrm{~K}$.

structure of the $\mathrm{NH}_{3}$ emission is consistent with gravitationally bound rotational motion of two clumps.

\section{A.20. V1057 Cyg}

V1057 Cyg belongs to the small group of the FU Orionis type stars. Before its flare-up in 1970 , it was a T Tauri star. A marginally resolved outflow was reported by Levreault (1989) and Evans et al. (1994). They detected only a blue wing extending to the north, but no contour map is shown.

We detected very weak ammonia emission toward this source (see Fig. 1 and Table 2) and the line analysis was carried out by averaging several positions, so no contour map could be made. This weak emission indicates either a low column density gas or that the $\mathrm{NH}_{3}$ emission is very compact. The lack of a large amount of high-density gas agrees with the fact that the source is optically visible.

\section{A.21. CB 232}

This Bok globule is associated with IRAS $21352+4307$, which is proposed to be the exciting source of a poorly collimated bipolar molecular outflow (Yun \& Clemens 1994a). The IRAS source has near-IR (Yun \& Clemens 1995) and millimeter counterparts (Launhardt \& Henning 1997), and is associated with two compact submillimeter sources. One of them, SMM1, is proposed as a Class 0 candidate (Huard et al. 1999).

We have detected an ammonia condensation (see Fig. A.25) unresolved by our beam, whose emission peak coincides with the position of the IRAS source. Given that the spectral energy distribution of the IRAS source is rising toward longer wavelengths, our results suggest that the IRAS source traces the location of one or several YSOs, deeply embedded in the high density gas, and that the globule is a site of very recent star formation.

\section{A.22. IC 1396E}

IC $1396 \mathrm{E}$ is a bright-rimmed cometary globule located in the northern periphery of the H II region IC 1396. Wilking et al. (1990) mapped a bipolar molecular outflow and proposed IRAS 21391+5802, an intermediate-mass YSO which is found roughly at the center of the globule, as the exciting source. This 
I. Sepúlveda et al.: Dense gas and the nature of the outflows

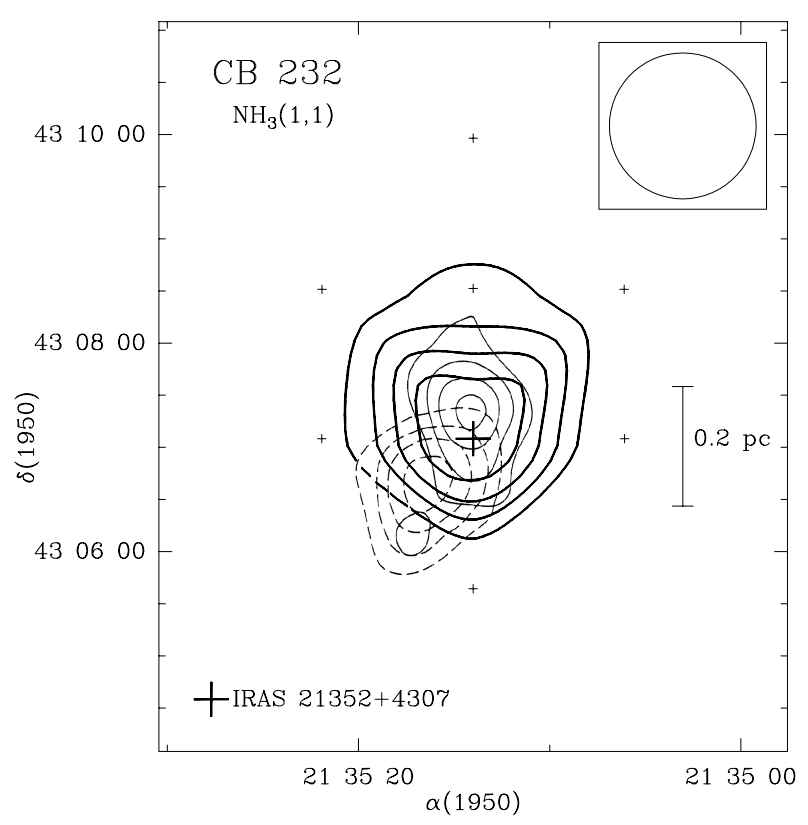

Fig. A.25. Same as Fig. A.1, for the CB 232 region. The $\mathrm{NH}_{3}$ lowest contour is $0.2 \mathrm{~K}$ and the increment is $0.1 \mathrm{~K}$. The $\mathrm{CO}$ bipolar outflow is from Yun \& Clemens (1994a).

IRAS source is detected at near-IR, submm, mm, and cm wavelengths (Wilking et al. 1993; Beltrán et al. 2002). The molecular outflow axis is oriented at a position angle of $75^{\circ}$, which is similar to the position angle of the $\mathrm{H}_{2} \mathrm{O}$ maser bipolar outflow observed at scales from 1 to $500 \mathrm{AU}$ (Patel et al. 2000).

The region was mapped in $\mathrm{NH}_{3}(1,1)$ and $\mathrm{NH}_{3}(2,2)$ (with an angular resolution of $40^{\prime \prime}$ ), and several other molecular lines by Serabyn et al. (1993). The $\mathrm{NH}_{3}$ clump mapped by these authors is elongated in the north-south direction and shows a temperature gradient increasing outward from the center and reaching a maximum on the surface most directly facing the stars ionizing IC1396.

Our $\mathrm{NH}_{3}$ map of the condensation (Fig. A.26), obtained with a poorer angular resolution, is elongated in the north-south direction, in good agreement with the one obtained by Serabyn et al. (1993). We found for this source a kinetic temperature of $\sim 19 \mathrm{~K}$, which is above the average for the sources studied in this paper. Although since we only have observed a single position in the $\mathrm{NH}_{3}(2,2)$ line we cannot establish the presence of the temperature gradient reported by Serabyn et al. (1993). The position of the source IRAS $21391+5802$ falls very close to the $\mathrm{NH}_{3}$ emission peak. This positional coincidence, as well as the spectral energy distribution of the source, suggest that it is a very young object, deeply embedded in the high density gas and the best candidate to drive the molecular outflow. There are two other IRAS sources in the region, but they lie outside, near the edge of the $\mathrm{NH}_{3}$ condensation (see Fig. A.26). At present, little is known about these sources and further studies are required to investigate their nature and relationship with the molecular condensation.

\section{A.23. $L 1165$}

L1165 is a small cloud whose distance is not well established. Estimates by different authors range from $200 \mathrm{pc}$ to $750 \mathrm{pc}$. We will adopt a distance of $750 \mathrm{pc}$, based on the assumption that the cloud is part of the IC 1396 region (Schwartz et al. 1991). Parker et al. (1991) discovered a bipolar molecular outflow

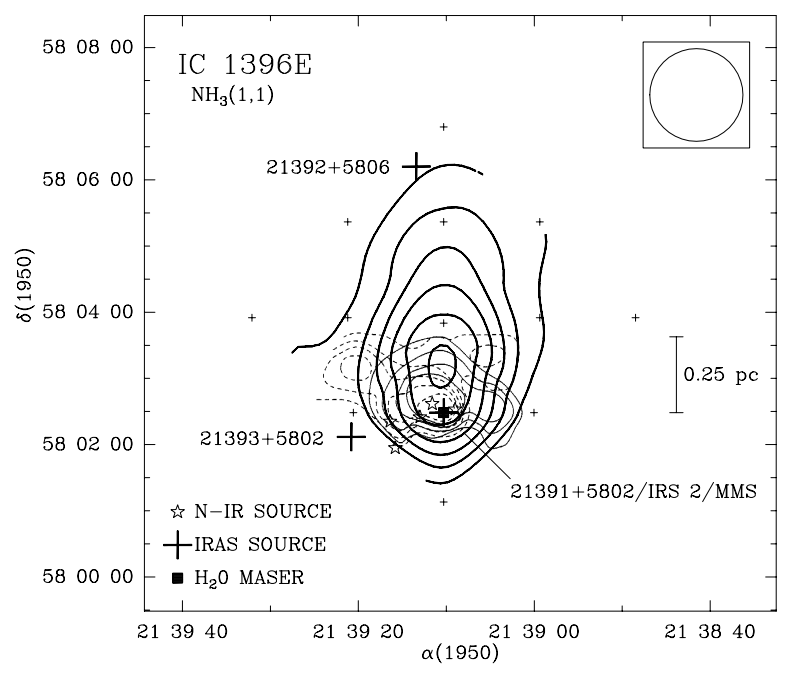

Fig. A.26. Same as Fig. A.1, but for the IC $1396 \mathrm{E}$ region. The $\mathrm{NH}_{3}$ lowest contour level is $0.2 \mathrm{~K}$ and the increment is $0.15 \mathrm{~K}$. The map of the CO bipolar outflow is from Wilking et al. (1990).

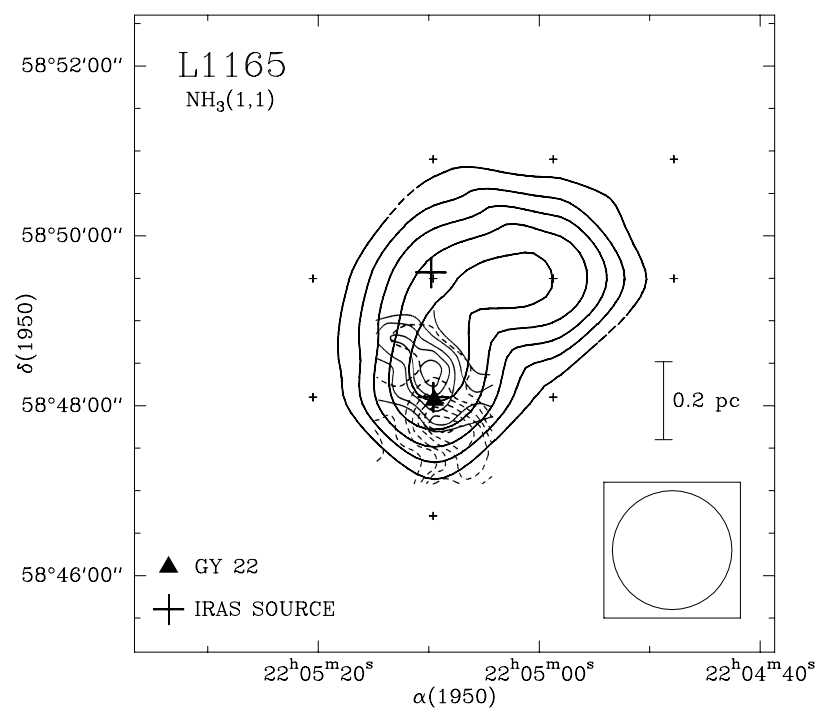

Fig. A.27. Same as Fig. A.1, for the $\mathrm{L} 1165$ region. The $\mathrm{NH}_{3}$ lowest contour is $0.15 \mathrm{~K}$ and the increment is $0.05 \mathrm{~K}$. The IRAS sources are, from north to south, IRAS $22051+5849$ and IRAS $22051+4858$ (=HH 354 IRS). The CO bipolar outflow is from Parker et al. (1991).

centered on IRAS $22051+5848$, which was proposed as the exciting source. This IRAS source, that has a near-IR counterpart (Tapia et al. 1997), is located $\sim 15^{\prime \prime}$ to the NE of the reflection nebulosity GY 22 (Gyulbudaghian 1982; Reipurth et al. 1997a). Reipurth et al. (1997a) reported a HH object, HH 354, located $11^{\prime} \mathrm{NE}$ of the IRAS position and at the end of a cavity in the molecular cloud, possibly excavated by the molecular outflow. These authors proposed that HH 354, the cavity, the molecular outflow and the GY 22 nebulosity are all parts of a single giant outflow excited by the IRAS source. From nearIR spectroscopy, Reipurth \& Aspin (1997) concluded that IRAS $22051+5848(=\mathrm{HH} 354$ IRS $)$ is a FUor candidate. The source IRAS $22051+5849$, which has also a near-IR counterpart (Tapia et al. 1997), lies $\sim 1.5$ north of IRAS $22051+5848$, well off the axis of the proposed giant outflow.

The $\mathrm{NH}_{3}$ map (Fig. A.27) shows a condensation with the emission peaking very close to the position of IRAS $22051+5848$. The IRAS colors of this source are typical 


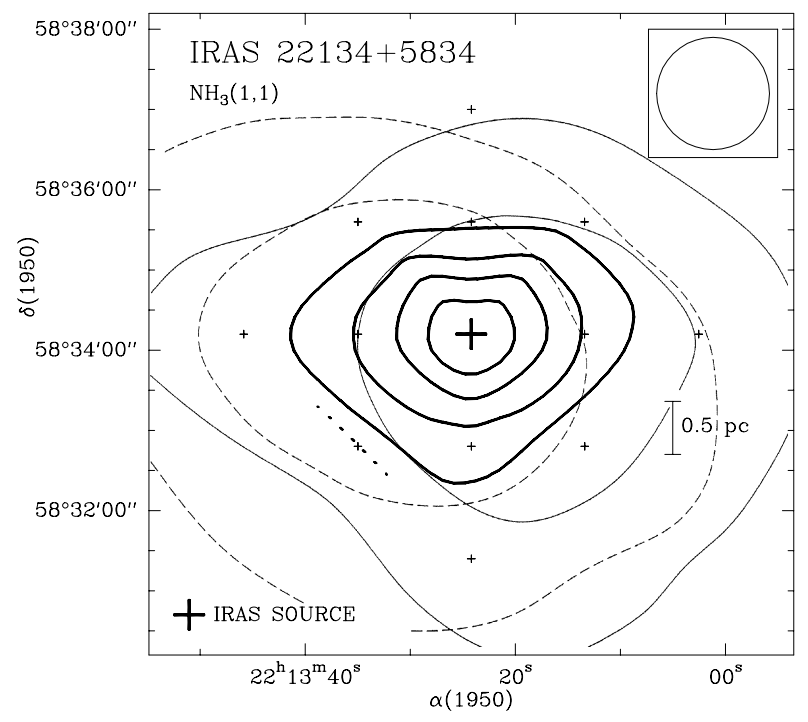

Fig. A.28. Same as Fig. A.1, for the region around IRAS 22134+5834. The $\mathrm{NH}_{3}$ lowest contour is $0.2 \mathrm{~K}$ and the increment is $0.1 \mathrm{~K}$. The $\mathrm{CO}$ bipolar outflow is from Dobashi et al. (1994).

of embedded sources (Parker 1991) and it is surrounded by the reflection nebulosity GY 22. Altogether suggest that IRAS $22051+5848$ is a young object embedded in the high density gas. IRAS $22051+5849$ is located close to the emission maximum and also appears associated with the dense gas, but its IRAS colors corresponding to a blackbody at $T>1000 \mathrm{~K}$ are suggestive of a background source (Tapia et al. 1997). This source needs more accurate observations in order to establish its relationship with the core and the outflow.

\section{A.24. IRAS $22134+5834$}

The distance of this source is established to be $2.6 \mathrm{kpc}$ (Sridharan et al. 2002), although previously a distance of 900 pc was assumed. Dobashi et al. (1994) discovered a molecular outflow associated with the source IRAS 22134+5834, one of the most luminous sources in the Cepheus region. $\mathrm{H}_{2}$ images (Kumar et al. 2002) revealed a dense stellar cluster around the IRAS source, that was interpreted as ring-shaped cluster (Kumar et al. 2003).

The $\mathrm{NH}_{3}$ distribution (Fig. A.28) shows a compact condensation with the emission maximum located at the IRAS position, in agreement with the ${ }^{12} \mathrm{CO}$ and ${ }^{13} \mathrm{CO}$ maps obtained by Dobashi et al. (1994). The IRAS source is bright at FIR wavelengths and not at NIR wavelengths. This and its association with high-density gas, suggest that IRAS $22134+5834$ is a very young stellar object, a possible massive protostar as suggested by Dobashi et al. (1994) and Kumar et al. (2003).

\section{A.25. $L 1221$}

L1221 is a small isolated dark cloud associated with IRAS 22266+6845, which has an energy distribution rising to longer wavelengths. Umemoto et al. (1991) discovered a bipolar molecular outflow centered near the position of the IRAS source. The outflow shows a U-shaped structure open to the northwest. However, CO observations with higher angular resolution showed that the outflow may consist of two bipolar outflows, an east-west outflow associated with the IRAS source and a north-south outflow originating about $25^{\prime \prime}$ to the east of the IRAS source (Lee et al. 2002).

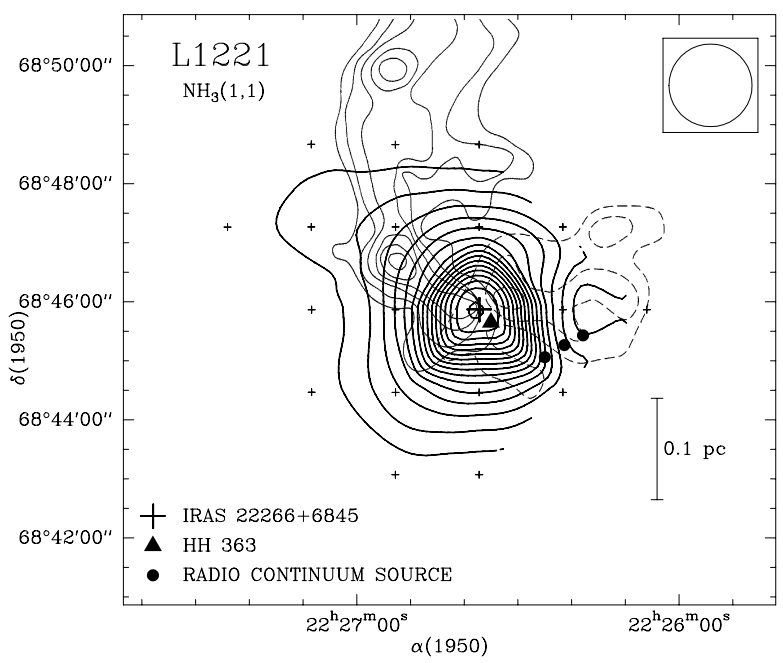

Fig. A.29. Same as Fig. A.1, for the L1221 region. The $\mathrm{NH}_{3}$ lowest contour level is $0.25 \mathrm{~K}$ and the increment is $0.2 \mathrm{~K}$. The map of the CO bipolar outflow is from Umemoto et al. (1991).

The region was mapped with different high-density tracer molecules (CS, $\mathrm{HCO}^{+}, \mathrm{HCN}, \mathrm{C}^{18} \mathrm{O}$; Umemoto et al. 1991, Lee $\&$ Ho 2005). Alten et al. (1997) discovered an HH object, HH 363, in the vicinity of the IRAS source. Anglada et al. (2005) detected three $\mathrm{cm}$ continuum sources in this region, but none seems to be associated with the IRAS source. Two mm continuum sources are detected, one of them (MM1) peaks around the IRAS source and toward one of the three infrared sources detected by the Spitzer Space Telescope (Lee \& Ho 2005)

The $\mathrm{NH}_{3}$ emission (Fig. A.29) is distributed in a compact condensation centered on the IRAS source, with weak emission extending to the NE. The size of the $\mathrm{NH}_{3}$ condensation (see Table 5) is similar to that obtained in the CS and $\mathrm{HCO}^{+}$maps (Umemoto et al. 1991), but the emission peaks of the $\mathrm{CS}, \mathrm{HCO}^{+}$, and HCN cores are located $\sim 54^{\prime \prime}(\sim 0.06 \mathrm{pc})$ to the south of the $\mathrm{NH}_{3}$ emission peak. A displacement between $\mathrm{CS}$ and $\mathrm{NH}_{3}$ emission peaks has been found in several other regions, and has been interpreted by Morata et al. (1997) in terms of chemical evolution.

The presence of the IRAS source toward the ammonia peak, which is located at the center of symmetry of the outflow, favors IRAS $22266+6845$ as its exciting source. This contrast with the proposal of Umemoto et al. (1991), who postulate the existence of an object located to the south of the position of the CS emission peak as the outflow driving source.

\section{A.26. NGC 7538}

The NGC 7538 molecular cloud is an active site of high-mass star formation containing five infrared sources (IRS 1, 2, 3, 9 and 11) within an area of 3'.5×3.5 (Werner et al. 1979). Estimates of the distance range from 2.2 to $4.7 \mathrm{kpc}$. We adopt a distance of 2.7 kpc (Kameya et al. 1986). Campbell \& Thompson (1984) found a high-velocity outflow near IRS 1. Kameya et al. (1989) discovered three additional outflows in the region. Two of them are associated with sources IRS 9 and IRS 11, but the third one was not associated with any known source. Davis et al. (1998) detected a collimated $\mathrm{H}_{2}$ jet associated with the IRS 9 outflow, two possible bow shocks related with the IRS 1 and IRS 9 outflows, and a number of $\mathrm{H}_{2}$ compact knots which coincide with the IRS 11 outflow, and that could be related with it. Davis et al. (1989) also detected a cavity to the northwest of IRS 1. 
I. Sepúlveda et al.: Dense gas and the nature of the outflows

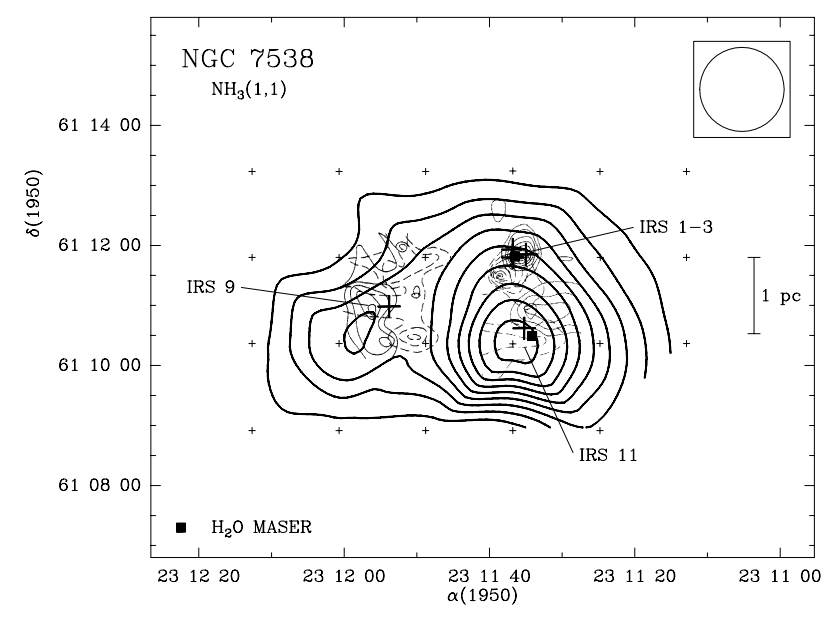

Fig. A.30. Same as Fig. A.1, for the NGC 7538 region. The $\mathrm{NH}_{3}$ lowest contour level is $0.25 \mathrm{~K}$ and the increment is $0.2 \mathrm{~K}$. The map of the $\mathrm{CO}$ bipolar outflows are from Kameya et al. (1989).

The $\mathrm{NH}_{3}$ map (Fig. A.30) shows a condensation elongated in the east-west direction. The ammonia emission peak is located toward the position of IRS 11. A secondary emission peak is located near IRS 9. IRS 1-3 also appear projected toward the ammonia condensation. The association of IRS 11 with the ammonia emission peak suggests that this source is the most embedded object, in agreement with the CS observations (Kameya et al. 1986).

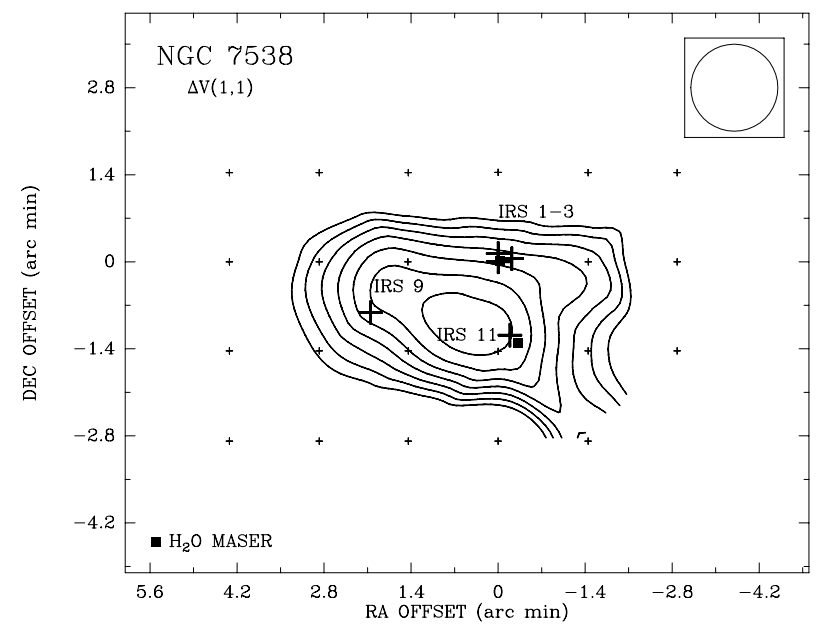

Fig. A.31. A contour map of the $\mathrm{NH}_{3}$ intrinsic line width for the NGC 7538 region. The lowest contour level is $1.6 \mathrm{~km} \mathrm{~s}^{-1}$ and the increment is $0.3 \mathrm{~km} \mathrm{~s}^{-1}$.

The $\mathrm{NH}_{3}$ line profiles show broad intrinsic line widths, with values ranging from $\Delta V=1.68 \mathrm{~km} \mathrm{~s}^{-1}$ to $3.41 \mathrm{~km} \mathrm{~s}^{-1}$. The broadest intrinsic line width is found very close to the IRS 11 position. Figure A.31 shows a contour map of the $\mathrm{NH}_{3}$ intrinsic line width. The broadening due to the $\mathrm{NH}_{3}$ hyperfine structure is about $0.5 \mathrm{~km} \mathrm{~s}^{-1}$ and the thermal broadening for the derived kinetic temperature $\left(\sim 28.3 \mathrm{~K}\right.$, see Table 5) is $\sim 0.27 \mathrm{~km} \mathrm{~s}^{-1}$. Both values are significantly lower than the observed line width, which could indicate turbulent motions of the gas, or that the dense gas is suffering an interaction with the outflows. 